Supporting Information for

\title{
4-Carboalkoxylated Polyvalerolactones from Malic Acid: Tough and Degradable Polyesters
}

Grant W. Fahnhorst, Guilhem X. De Hoe, Marc A. Hillmyer, Thomas R. Hoye* Department of Chemistry, University of Minnesota, Minneapolis, Minnesota 55455, USA

* hoye@umn.edu

\section{Contents of Supporting Information}

page \#

I. General Experimental Protocols $\quad$ S2-S3

II. Preparation and Characterization of Small Molecules S4-S20

IIa. Coumalates

S4-S9

IIb. 4-Carboalkoxyvalerolactones (CRVLs) and 5-Alkoxy-4-methyl-5-oxopentanoic acids S10-20

$\begin{array}{lr}\text { III. Preparation and Characterization of Polymers } & \text { S21-S29 }\end{array}$

IV. Polymer Testing $\quad$ S30-S37

IVa. Matrix-assisted laser desorption ionization time of flight (MALDI-TOF) S30

$\begin{array}{ll}\mathrm{IVb} \text {. DBU-promoted eliminative degradation } & \text { S31-S32 }\end{array}$

IVc. Differential scanning calorimetry (DSC) annealing studies $\quad$ S33-S37

V. Polymer Characterization Data $\quad$ S38-S49

$\begin{array}{ll}\text { Va. Size-exclusion chromatography (SEC) S38-S40 } & \text { S3) }\end{array}$

$\begin{array}{ll}\text { Vb. Thermogravimetric analysis (TGA) } & \text { S41-S42 }\end{array}$

Vc. Differential scanning calorimetry (DSC) S43-S44

$\begin{array}{ll}\text { Vd. Tensile testing } & \text { S45-S47 }\end{array}$

$\begin{array}{ll}\text { Ve. Hydrolytic degradation } & \text { S48-S49 }\end{array}$

$\begin{array}{ll}\text { VI. References } & \text { S50 }\end{array}$

VII. Copies of ${ }^{1} \mathrm{H}$ and ${ }^{13} \mathrm{C}$ NMR Spectra $\quad$ S50-S97 


\section{General Experimental Protocols}

\section{Materials:}

D,L-Malic acid ( $\geq 99 \%$, Sigma Aldrich or Acros Organics), benzyl bromide (Aldrich), undecyl bromide (Alfa Aesar), 2-ethylhexyl bromide (Aldrich), and Pd/C [5\% (w/w), Engelhard] were used as received. $\mathrm{BnOH}$ was distilled over $\mathrm{CaH}_{2}$ and stored under a $\mathrm{N}_{2}$ atmosphere in a glovebox. Diphenyl phosphate [DPP; $\left.(\mathrm{PhO})_{2} \mathrm{PO}_{2} \mathrm{H}\right]$, from Ark Pharm or Sigma Aldrich, was placed in a vial containing a stir bar, and the sample was stirred under high vacuum (ca. $0.1 \mathrm{~mm}$ $\mathrm{Hg}$ ) for $48 \mathrm{~h}$ before use. Solvents were used as received unless otherwise noted.

\section{Instrumental methods:}

NMR: Bruker Avance spectrometers $(500 \mathrm{MHz}$ and $400 \mathrm{MHz})$ were used to obtain ${ }^{1} \mathrm{H}$ and ${ }^{13} \mathrm{C}$ NMR spectral data. Chemical shifts are referenced to $\delta 2.50 \mathrm{ppm}\left(\mathrm{CHD}_{2} \mathrm{SOCD}_{3}\right)$ for ${ }^{1} \mathrm{H} \mathrm{NMR}$ spectra or $\delta 39.56 \mathrm{ppm}\left[\left(\mathrm{CD}_{3}\right)_{2} \mathrm{SO}\right]$ for ${ }^{13} \mathrm{C}$ spectra for spectra taken in DMSO- $d_{6}$. Chemical shifts for spectra recorded in $\mathrm{CDCl}_{3}$ are referenced to $\delta 0.00 \mathrm{ppm}$ (internal TMS) for ${ }^{1} \mathrm{H} \mathrm{NMR}$ spectra or $\delta 77.16 \mathrm{ppm}\left[\mathrm{CDCl}_{3}\right.$ resonance] for ${ }^{13} \mathrm{C}$ spectra. Coupling constant values were determined using methods described previously. ${ }^{1}$ The chemical shift of each proton resonance is reported in ppm followed immediately, in parentheses, by the [multiplicity, coupling constant(s), relative number of protons (to the nearest whole proton), and the substructure environment of the proton]. Assignment of the substructural environments are indicated by italicization of the specific proton (e.g., $\mathrm{CH}_{a} \mathrm{H}_{b}$ ). Non-first-order multiplets are identified with the acronym 'nfom.'

ATR-FTIR: Sixteen scans and a four second acquisition time were used to obtain FT-IR spectra on a Bruker Alpha Platinum FTIR instrument containing an ATR single-bounce diamond crystal.

Mass spectrometry of non-polymeric samples: High resolution mass spectra (HRMS) of the lactone monomers were recorded using a Bruker BioTOF (ES-TOF) instrument. PEG was used as an internal calibrant/standard. HRMS data for other small molecule samples were obtained using a Thermo Orbitrap Velos spectrometer using electrospray ionization (ESI) or atmospheric pressure chemical ionization (APCI) in the positive ion mode. Pierce ${ }^{\mathrm{TM}}$ LTQ was used as an external calibration standard.

Size Exclusion Chromatography (SEC): An Agilent 1260 Series pump was fitted with an Optilab rEX differential refractive index detector, a Wyatt 18-angle DAWN HELEOS light scattering detector, and two serial Agilent PLgel MIXED-B columns (300 mm L x $7.5 \mathrm{~mm}$ ID packed with $10 \mu \mathrm{m}$ beads). Matrix-assisted laser light scattering data (MALS) were used to determine $M_{\mathrm{n}}$, $M_{\mathrm{w}}$, and $D$ of samples.

Thermogravimetric Analysis (TGA): Polymeric samples (4-10 mg) were analyzed using a TA Instruments Q500 instrument. Each sample was heated at a rate of $10{ }^{\circ} \mathrm{C} \mathrm{min}^{-1}$ under $\mathrm{N}_{2}$ unless otherwise noted. The mass loss of $1 \%$ and $5 \%$ are reported for each sample.

Differential Scanning Calorimetry (DSC): Polymeric samples were sealed hermetically in aluminum pans under atmospheric conditions prior to DSC analysis using a TA Instruments Q$1000 \mathrm{DSC}$. The lower molecular weight samples were heated from ambient temperature to 150 ${ }^{\circ} \mathrm{C}\left(10^{\circ} \mathrm{C} \mathrm{min}{ }^{-1}\right)$, cooled to $-60{ }^{\circ} \mathrm{C}\left(10^{\circ} \mathrm{C} \mathrm{min}^{-1}\right)$, heated again to $150{ }^{\circ} \mathrm{C}\left(10^{\circ} \mathrm{C} \mathrm{min}^{-1}\right)$, cooled to $-60{ }^{\circ} \mathrm{C}\left(5^{\circ} \mathrm{C} \mathrm{min}^{-1}\right)$, and heated to $150{ }^{\circ} \mathrm{C}\left(5^{\circ} \mathrm{C} \mathrm{min}^{-1}\right)$. The reported glass transition, crystallization, and melting temperatures values are those from the final $\left(5^{\circ} \mathrm{C} \mathrm{min}{ }^{-1}\right)$ heating cycle unless otherwise noted. 
Matrix-Assisted Laser Desorption/Ionization Time of Flight Mass Spectrometry (MALDI/TOF$M S$ ): A MALDI instrument (AB-Sciex 5800) set in reflectron mode was used to obtain MALDI/TOF-MS spectra of PCRVL samples having $M_{\mathrm{n}}=\mathrm{ca} .2500 \mathrm{~g} \mathrm{~mol}^{-1}$. The PCRVL samples were dissolved in THF. Dihydroxybenzene was used as the matrix and dissolved in THF also. The matrix and polymer were mixed in a mass ratio of ca. 2000:1 and applied to the target plate and the THF allowed to evaporate under ambient conditions before insertion into the instrument.

Tensile Testing: High molar mass samples $\left[M_{\mathrm{w}} 65-130 \mathrm{~kg} \mathrm{~mol}^{-1}\right.$ (MALS)] of PCMeVL, PCEtVL, PCiPrVL, and PCnBuVL were melt-pressed at $120^{\circ} \mathrm{C}$ for five minutes between two Teflon sheets using a square mold with a thickness of $0.2 \mathrm{~mm}$. Each sample was annealed for a minimum $72 \mathrm{~h}$ prior to testing. A dog-bone-shaped die was used to punch out samples for tensile testing; each sample had a $0.2 \mathrm{~mm}$ thickness, ca. $20 \mathrm{~mm}$ gauge length, $5 \mathrm{~mm}$ gauge width, and $15 \mathrm{~mm}$ clamp width. Samples were pulled to the point of break with a rate of $50.0 \mathrm{~mm} \mathrm{~min}^{-1}$ using a Shimadzu AGS-X Tensile Tester.

Hydrolytic Degradation: Residual samples of the high molar mass polymers $\left(M_{\mathrm{w}} 65-130 \mathrm{~kg} \mathrm{~mol}^{-}\right.$ ${ }^{1}$ ) of PCMeVL, PCEtVL, PCiPrVL, and PCnBuVL used in the above tensile testing studies were melt pressed at $120^{\circ} \mathrm{C}$ for five minutes between two Teflon sheets and a square mold with a thickness of $0.2 \mathrm{~mm}$. The polymers were then annealed at room temperature until DSC analysis indicated that the degree of crystallinity of each polymer remained unchanged (minimum $72 \mathrm{~h}$ annealing time). Square samples (side lengths were between 12 and $14 \mathrm{~mm}$; approximate mass per sample was $35 \mathrm{mg}$ ) were cut from each sheet of polymer and immersed in one of three aqueous solutions: 0.1 M NaOH, 0.1 M HCl, or 0.1 M phosphate-buffered saline (PBS, pH 7.4). Each solution was made using HPLC grade water (Fisher Chemical). The degradation experiments were performed using $13 \mathrm{~mL}$ of degradation medium in $15 \mathrm{~mL}$ polypropylene centrifuge tubes (Corning 430790), and each experiment was performed in triplicate. Control experiments with no polymer (i.e., just $13 \mathrm{~mL}$ of degradation media) were performed in parallel. One set of experiments was performed at elevated temperature $\left(80^{\circ} \mathrm{C}\right.$, above the $T_{\mathrm{m}}$ of all polymers studied) and one set of experiments was performed at room temperature. At various timepoints, $200 \mu \mathrm{L}$ aliquots were removed from each centrifuge tube and transferred to $1.7 \mathrm{~mL}$ microcentrifuge tubes, which were then stored at $4{ }^{\circ} \mathrm{C}$ until further analysis. Prior to removing each aliquot, the centrifuge tubes were mixed using a vortex mixer to ensure accurate sampling of the degradation media. Centrifugation was also used prior to sampling (but after vortex mixing) if any polymer particles were dispersed in the solution; this ensured no solid particles were present in the aliquots. The aliquots were analyzed using a total organic carbon analyzer (Shimadzu TOC-L) by removing $80 \mu \mathrm{L}$ from the microcentrifuge tube and diluting to $8 \mathrm{~mL}$. The total organic carbon (TOC) content of each aliquot was then calculated using a calibration curve (0-40 ppm of C) obtained using potassium hydrogen phthalate (Sigma P1088) as a calibrant. The TOC content of the control samples was subtracted from the TOC content measured for each aliquot. The corrected TOC content of each aliquot was then compared to the amount of carbon present in the initial amount of polymer to obtain a $\% \mathrm{C}$; the average $\% \mathrm{C}$ for each triplicate was then plotted as a function of time. 


\section{Preparation and Characterization of Small Molecules}

IIa. Coumalates

\section{Coumalic Acid}

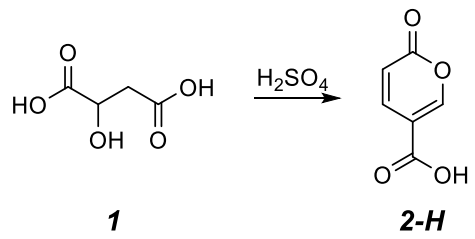

Coumalic acid was prepared using a modified procedure to Wiley and Smith. ${ }^{2}$ Malic acid (400 g, $2.99 \mathrm{~mol}$ ) was added portion-wise (ca. $25 \mathrm{~g} / 10 \mathrm{~min})$ to sulfuric acid $(1 \mathrm{~L})$ at $65^{\circ} \mathrm{C}$ in a 3-L round bottom flask for $20 \mathrm{~h}$. The contents were poured over ice (ca. $1.5 \mathrm{~L})$, and the mixture was allowed to stand for $24 \mathrm{~h}$. The mixture was filtered, and the filtered solid was thoroughly washed with cold water to afford coumalic acid as a tan powder (113 g. 54\% yield). This powder was commonly 97-98\% pure as determine by ${ }^{1} \mathrm{H}$ NMR spectroscopy and was often further purified by recrystallization in $\mathrm{MeOH}$ to give coumalic acid (2-H, $76.5 \mathrm{~g}, 37 \%$ yield) as a tan solid.

mp onset of sublimation at $170{ }^{\circ} \mathrm{C}, \mathrm{mp}=194-202{ }^{\circ} \mathrm{C}\left(\right.$ lit. $\left.206-209{ }^{\circ} \mathrm{C}\right) .^{2}$

\section{Coumalic Acid Chloride (S1)}

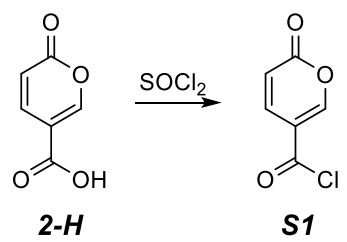

Coumalic acid (2-H, $2.79 \mathrm{~g}, 19.9 \mathrm{mmol})$ was refluxed in thionyl chloride (11.5 mL, $159 \mathrm{mmol})$ in a $25-\mathrm{mL}$ round bottom flask until the solid was completely consumed (24-40 h). The reaction mixture was concentrated and distilled via bulb-to-bulb distillation to give coumalic acid chloride (S1, $2.83 \mathrm{~g}, 17.9 \mathrm{mmol}, 90 \%$ yield) as a white solid. Because this compound degrades at room temperature in a day or so to a dark brown solid (sparingly soluble in $\mathrm{CHCl}_{3}$ ) it was routinely used immediately after preparation.

${ }^{1} \mathbf{H}$ NMR $\left(500 \mathrm{MHz}, \mathrm{CDCl}_{3}\right) \delta 8.58,(\mathrm{dd}, J=2.8,1.1 \mathrm{~Hz}, 1 \mathrm{H},(\mathrm{CO}) \mathrm{O}-\mathrm{CH}=\mathrm{C}), 7.77$ (dd, $J=9.9$, $2.8 \mathrm{~Hz},-\mathrm{CHCH}(\mathrm{C}=\mathrm{O})-)$, and 6.39 (dd, $J=9.9,1.1 \mathrm{~Hz}, 1 \mathrm{H},-\mathrm{CHCH}(\mathrm{C}=\mathrm{O})-)$.

${ }^{13} \mathbf{C}$ NMR $\left(125 \mathrm{MHz}, \mathrm{CDCl}_{3}\right) \delta$ 163.0, 162.8, 158.3, 140.3, 116.7, and 115.5.

IR 3089, 3065, 3035, 1732 (strong, with two shoulders), 1628 (med), 1556 (med), and 1267 (strong) $\mathrm{cm}^{-1}$.

bp $105-115^{\circ} \mathrm{C}$ at $0.15 \mathrm{mmHg}$ 


\section{Methyl Coumalate (2-Me)}

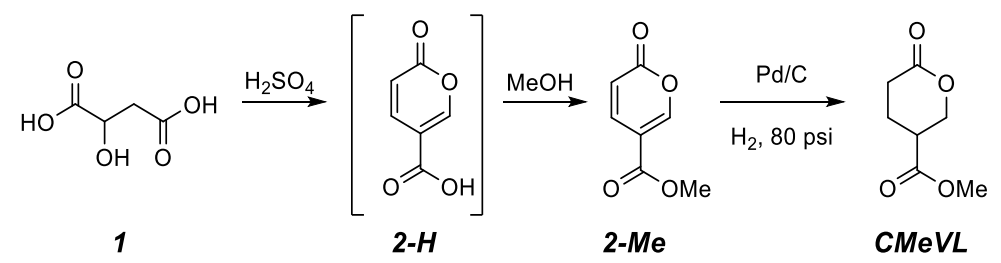

Methyl coumalate (2-Me) and 4-carbomethoxyvalerolactone $(\mathbf{C M e V L})$ were synthesized by a procedure we previously described. ${ }^{3}$ 


\section{Ethyl Coumalate (2-Et)}

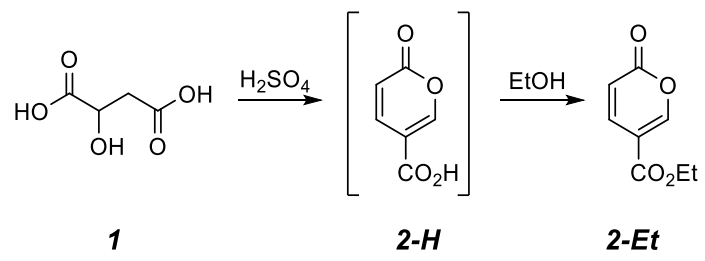

Ethyl coumalate (2-Et) was synthesized using an analogous procedure to that used for methyl coumalate. ${ }^{3}$ D,L-Malic acid $(1,200.0 \mathrm{~g}, 745.7 \mathrm{mmol})$ was added portionwise (ca. $20 \mathrm{~g}$ /addition) over the course of one hour to a stirred solution of sulfuric acid $(500 \mathrm{~mL})$ in a 3-liter roundbottomed flask at $70{ }^{\circ} \mathrm{C}$. The mixture was allowed to stir for $16 \mathrm{~h}$, at which time the color had progressed to dark red. This mixture was placed into an ice bath. Once the internal temperature of the mixture reached $35-40{ }^{\circ} \mathrm{C}$, ethanol $(700 \mathrm{~mL})$ was added dropwise at a rate to keep the reaction mixture between $35-50{ }^{\circ} \mathrm{C}(\mathrm{ca} .1 .5 \mathrm{~h})$. Note: when the mixture was cooled further than $30-35^{\circ} \mathrm{C}$ a precipitate, presumably coumalic acid, would begin to appear. Also, the addition of ethanol can generate a significant amount of heat. The solution was heated to $65{ }^{\circ} \mathrm{C}$ and stirred for $18 \mathrm{~h}$. The solution was cooled and poured over ice (ca. $1.2 \mathrm{~L}$ ), extracted with $\mathrm{CHCl}_{3}$, washed $\left(\mathrm{NaHCO}_{3}\right.$ and brine), dried $\left(\mathrm{MgSO}_{4}\right)$, and concentrated to give a yellow liquid. The crude ethyl coumalate was distilled via bulb-to-bulb distillation $(0.1 \mathrm{~mm} \mathrm{Hg}$, external bath temperature at $120{ }^{\circ} \mathrm{C}$ ) with the first ca. $10 \%$ discarded. This fraction contained significant quantities of diethyl fumarate and diethyl maleate. The main fraction of distilled coumalate $(76.0 \mathrm{~g})$ was recrystallized (EtOAc:Hex, slowly cooled to $-20^{\circ} \mathrm{C}$ ) to give ethyl coumalate (2-Et, $45.9 \mathrm{~g}, 273$ mmol, 37\% yield) as a white powder. The concentrated mother liquor could be distilled to provide additional ethyl coumalate.

${ }^{1} \mathbf{H}$ NMR $\left(500 \mathrm{MHz}, \mathrm{CDCl}_{3}\right) \delta 8.30(\mathrm{dd}, J=2.6,1.1 \mathrm{~Hz}, 1 \mathrm{H},(\mathrm{CO}) \mathrm{O}-\mathrm{CH}=\mathrm{C}), 7.80$ (dd, $J=9.8$, $2.6 \mathrm{~Hz}, 1 \mathrm{H},-\mathrm{CHCH}(\mathrm{CO})-), 6.34$ (dd, $J=9.8,1.1 \mathrm{~Hz}, 1 \mathrm{H},-\mathrm{CHCH}(\mathrm{C}=\mathrm{O})-), 4.34$ (q, J = 7.1 Hz, $\left.2 \mathrm{H},-\mathrm{CH}_{2} \mathrm{CH}_{3}\right)$, and $1.36\left(\mathrm{t}, J=7.1 \mathrm{~Hz}, 3 \mathrm{H},-\mathrm{CH}_{2} \mathrm{CH}_{3}\right)$.

${ }^{13} \mathrm{C}$ NMR $\left(125 \mathrm{MHz}, \mathrm{CDCl}_{3}\right) \delta 163.1,160.0,158.0,141.9,115.3,112.2,61.8$, and 14.4.

IR (neat, selected peaks): 3085, 3074, 2940, 1764 (med), 1745 (strong), 1698 (strong), 1634 (med), and 1556 (med) $\mathrm{cm}^{-1}$.

HRMS Calculated for $\left(\mathrm{C}_{8} \mathrm{H}_{9} \mathrm{O}_{4}\right)^{+}$169.0495; found: 169.0491 .

bp (bulb-to-bulb external temp): $120{ }^{\circ} \mathrm{C} @ 0.1 \mathrm{mmHg}$.

mp $33-36{ }^{\circ} \mathrm{C}\left(\right.$ lit. $\left.=35^{\circ} \mathrm{C}\right) .{ }^{4}$ 


\section{Isopropyl Coumalate (2-iPr)}

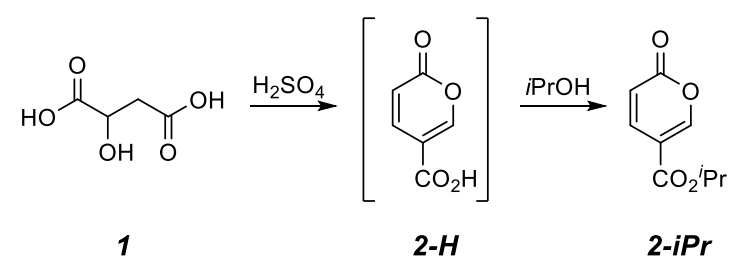

Isopropyl coumalate (2-iPr) was synthesized using a procedure analogous to that was used to prepare methyl coumalate. ${ }^{3}$ D,L-Malic acid (1, $200.0 \mathrm{~g}, 745.7 \mathrm{mmol}$ ) was added portionwise (ca. $20 \mathrm{~g}$ /addition) over the course of 90 minutes to a stirred solution of sulfuric acid $(500 \mathrm{~mL})$ in a 3 liter round-bottomed flask at $70{ }^{\circ} \mathrm{C}$. The mixture was allowed to stir for $12 \mathrm{~h}$, at which time the color had progressed to dark red. The mixture was placed into an ice bath. Once the internal temperature of the mixture reached $35-40{ }^{\circ} \mathrm{C}$, isopropanol $(950 \mathrm{~mL})$ was added dropwise at a rate to keep the reaction mixture between $35-50{ }^{\circ} \mathrm{C}(\mathrm{ca} .1 .5 \mathrm{~h})$. Note: when the mixture was cooled further than $30-35^{\circ} \mathrm{C}$ a precipitate, presumably coumalic acid, began to appear. The addition of $i$-PrOH generates a significant amount of heat. The solution was heated to $65{ }^{\circ} \mathrm{C}$ and stirred for $24 \mathrm{~h}$. The solution was cooled and poured over ice (ca. 1.2 L), extracted with $\mathrm{CHCl}_{3}$, washed $\left(\mathrm{NaHCO}_{3}\right.$ and brine), dried $\left(\mathrm{MgSO}_{4}\right)$, and concentrated to give a yellow solid. The crude coumalate was distilled via bulb-to-bulb distillation $(0.1 \mathrm{~mm} \mathrm{Hg}$, external bath temperature at $120{ }^{\circ} \mathrm{C}$ ) with the first ca. $10 \%$ discarded. This fraction contained significant quantities of the diisopropyl fumarate and diisopropyl maleate. The distilled coumalate $(74.5 \mathrm{~g})$ was recrystallized (pet ether) to give isopropyl coumalate (2-iPr, $49.7 \mathrm{~g}, 273 \mathrm{mmol}, 37 \%$ yield) as white, needlelike crystals. The concentrated mother liquor could be distilled to recover additional isopropyl coumalate.

${ }^{1} \mathbf{H}$ NMR $\left(500 \mathrm{MHz}, \mathrm{CDCl}_{3}\right) \delta 8.28(\mathrm{dd}, J=2.6,1.1 \mathrm{~Hz}, 1 \mathrm{H},(\mathrm{CO}) \mathrm{O}-\mathrm{CH}=\mathrm{C}), 7.79(\mathrm{dd}, J=9.8$, $2.6 \mathrm{~Hz}, 1 \mathrm{H},-\mathrm{CHCH}(\mathrm{C}=\mathrm{O})-), 6.34(\mathrm{dd}, J=9.8,1.1 \mathrm{~Hz}, 1 \mathrm{H},-\mathrm{CHCH}(\mathrm{C}=\mathrm{O})-), 5.21$ (septet, $J=6.3$ $\left.\mathrm{Hz}, 1 \mathrm{H},-\mathrm{CH}\left(\mathrm{CH}_{3}\right)_{2}\right)$, and $1.34\left(\mathrm{~d}, \mathrm{~J}=6.3 \mathrm{~Hz}, 6 \mathrm{H},-\mathrm{CH}\left(\mathrm{CH}_{3}\right)_{2}\right)$.

${ }^{13}$ C NMR (125 MHz, $\left.\mathrm{CDCl}_{3}\right) \delta$ 162.6, 160.1, 158.0, 142.0, 115.3, 112.7, 69.6, and 22.0.

IR (neat, selected peaks): 3091, 2985, 1748 (strong), 1702 (strong), 1637 (med), 1556 (med), and 1429 (med) $\mathrm{cm}^{-1}$.

bp (bulb-to-bulb external temp): $120^{\circ} \mathrm{C} @ 0.1 \mathrm{mmHg}$.

mp $44-47^{\circ} \mathrm{C}\left(\right.$ lit. $\left.=44{ }^{\circ} \mathrm{C}\right) .^{5}$

HRMS Calculated for $\left(\mathrm{C}_{9} \mathrm{H}_{11} \mathrm{O}_{4}\right)^{+} 183.0652$; found: 183.0647 . 
n-Butyl Coumalate (2-nBu)

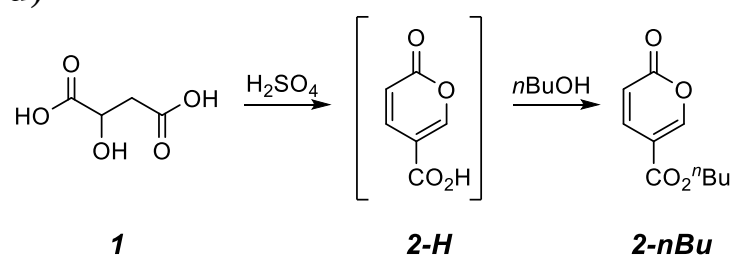

$n$-Butyl coumalate was synthesized using an analogous procedure to methyl coumalate. ${ }^{3} \mathrm{D}, \mathrm{L}-$ Malic acid (1, $200.0 \mathrm{~g}, 745.7 \mathrm{mmol}$ ) was added portionwise (ca. $20 \mathrm{~g} /$ addition) over the course of one to two hours to a stirred solution of sulfuric acid $(500 \mathrm{~mL})$ in a 3-liter round bottom flask at $70{ }^{\circ} \mathrm{C}$. The mixture was allowed to stir for $12 \mathrm{~h}$, at which time the color had progressed to dark red. The mixture was placed into an ice bath. Once the internal temperature of the mixture reached $35-40{ }^{\circ} \mathrm{C}, n$-butanol $(700 \mathrm{~mL})$ was added dropwise at a rate to keep the reaction mixture between $35-50{ }^{\circ} \mathrm{C}$ (ca. $1.5 \mathrm{~h}$ ). Note: when the mixture was cooled further than $30-35{ }^{\circ} \mathrm{C} \mathrm{a}$ precipitate, presumably coumalic acid, appeared. The addition of n-butanol generates a significant amount of heat. The solution was heated to $65^{\circ} \mathrm{C}$ and stirred for $8 \mathrm{~h}$. The solution was cooled and poured over ice (ca. $1.5 \mathrm{~L}$ ), extracted with $\mathrm{CHCl}_{3}$, washed $\left(\mathrm{NaHCO}_{3}\right.$ and brine), dried $\left(\mathrm{MgSO}_{4}\right)$, and concentrated to give a yellow solid. The crude coumalate was distilled via bulb-to-bulb distillation $\left(0.05 \mathrm{~mm} \mathrm{Hg}\right.$, external bath temperature at $\left.130{ }^{\circ} \mathrm{C}\right)$ with the first ca. $10 \%$ discarded. This fraction contained significant quantities of the dibutyl fumarate and dibutyl maleate. The distilled coumalate was recrystallized (pet ether) to give $n$-butyl coumalate (2-nBu, $86.7 \mathrm{~g}, 442 \mathrm{mmol}, 59 \%$ yield) as white crystals. The concentrated mother liquor could be distilled to recover additional $n$-butyl coumalate.

${ }^{1} \mathbf{H}$ NMR $\left(500 \mathrm{MHz}, \mathrm{CDCl}_{3}\right) \delta 8.30(\mathrm{dd}, J=2.6,1.1 \mathrm{~Hz}, 1 \mathrm{H},(\mathrm{CO}) \mathrm{O}-\mathrm{CH}=\mathrm{C}), 7.80(\mathrm{dd}, J=9.8$, $2.6 \mathrm{~Hz}, 1 \mathrm{H},-\mathrm{CHCHCO}), 6.34(\mathrm{dd}, J=9.8,1.1 \mathrm{~Hz}, 1 \mathrm{H},-\mathrm{CHCH}(\mathrm{C}=\mathrm{O})-), 4.29$ (t, $J=6.7 \mathrm{~Hz}, 2 \mathrm{H}$, $\left.-(\mathrm{CO}) \mathrm{O}-\mathrm{CH}_{2} \mathrm{CH}_{2}\right), 1.71\left(\mathrm{tt}, J=7.6,6.7 \mathrm{~Hz}, 2 \mathrm{H},-\mathrm{CH}_{2} \mathrm{CH}_{2} \mathrm{CH}_{2} \mathrm{CH}_{3}\right), 1.44(\mathrm{qd}, J=7.5,7.5 \mathrm{~Hz}, 2 \mathrm{H}$, $\left.-\mathrm{CH}_{2} \mathrm{CH}_{2} \mathrm{CH}_{2} \mathrm{CH}_{3}\right)$, and $0.97\left(\mathrm{t}, \mathrm{J}=7.5 \mathrm{~Hz}, 3 \mathrm{H},-\mathrm{CH}_{2} \mathrm{CH}_{3}\right)$.

${ }^{13} \mathrm{C}$ NMR $\left(125 \mathrm{MHz}, \mathrm{CDCl}_{3}\right) \delta 163.1,160.0,158.1,141.8,115.4,112.3,65.6,30.7,19.3$, and 13.8 .

IR (neat, selected peaks): 3090, 2965, 2934, 2873, 1712 (br, strong), 1637 (med), 1553, 1429 (med) $\mathrm{cm}^{-1}$.

bp $0.05 \mathrm{~mm} \mathrm{Hg}$, external bath temperature at $130{ }^{\circ} \mathrm{C}$.

mp $40-42{ }^{\circ} \mathrm{C}\left(\right.$ lit. $\left.=41^{\circ} \mathrm{C}\right) .^{5}$

HRMS Calculated for $\left(\mathrm{C}_{10} \mathrm{H}_{13} \mathrm{O}_{4}\right)^{+}$197.0808; found: 197.0804 . 


\section{t-Butyl Coumalate (2-tBu)}

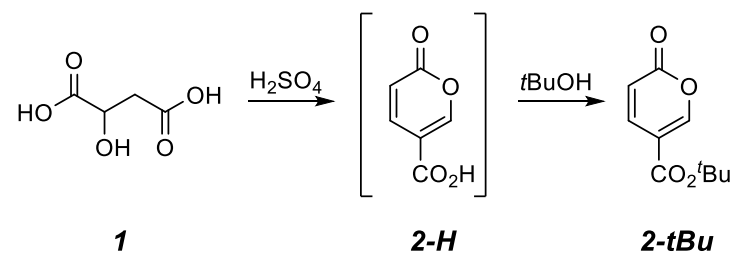

$t$-Butyl coumalate (2-tBu) was prepared as described by Yamashita et al. ${ }^{6}$ Yields ranged from $10-35 \%$, tending to be lower on larger scales $(>10 \mathrm{~g})$.

${ }^{1} \mathbf{H}$ NMR $\left(500 \mathrm{MHz}, \mathrm{CDCl}_{3}\right) \delta 8.21(\mathrm{dd}, J=2.6,1.1 \mathrm{~Hz}, 1 \mathrm{H},(\mathrm{CO}) \mathrm{O}-\mathrm{CH}=\mathrm{C}), 7.75(\mathrm{dd}, J=9.8$, $2.6 \mathrm{~Hz}, 1 \mathrm{H},-\mathrm{CHCHCO}), 6.32(\mathrm{dd}, J=9.8,1.1 \mathrm{~Hz}, 1 \mathrm{H},-\mathrm{CHCH}(\mathrm{C}=\mathrm{O})-), 1.55\left(\mathrm{~s}, 9 \mathrm{H},-\mathrm{C}\left(\mathrm{CH}_{3}\right)_{3}\right.$.

${ }^{13} \mathrm{C}$ NMR (125 MHz, $\mathrm{CDCl}_{3}$ ) $\delta$ 162.0, 160.2, 157.6. 142.0, 115.0, 113.4, 82.7, and 28.1.

IR (neat, selected peaks) 3085, 2983, 2974, 1754 (strong), 1728 (med), 1706 (strong), 1622 (strong), 1576 (strong), and $1439 \mathrm{~cm}^{-1}$.

HRMS Calculated for $\left(\mathrm{C}_{10} \mathrm{H}_{13} \mathrm{O}_{4}\right)^{+}$197.0808; found: 197.0803 .

mp $64-69^{\circ} \mathrm{C}$. 
IIb. 4-Carboalkoxyvalerolactones (CRVLs) and 5-Alkoxy-4-methyl-5-oxopentanoic acids

4-Carboethoxyvalerolactone (Ethyl 6-oxotetrahydro-2H-pyran-3-carboxylate, CEtVL) and 5-Ethoxy-4-methyl-5-oxopentanoic acid (S2)

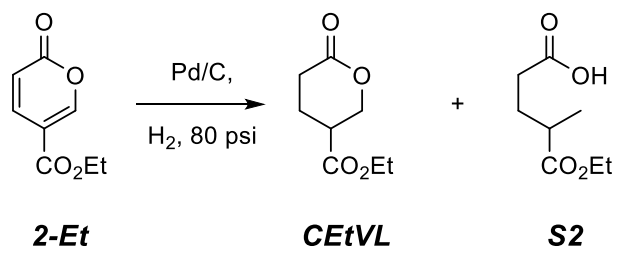

Ethyl coumalate (2-Et, $40.0 \mathrm{~g}, 238 \mathrm{mmol})$ and $\mathrm{Pd} / \mathrm{C}[3.0 \mathrm{~g}, 5 \% \mathrm{Pd} / \mathrm{C}]$ were placed into and sealed in a $500-\mathrm{mL}$ Fisher-Porter vessel ${ }^{8}$. THF $(250 \mathrm{~mL})$ was added by being poured down the side of the reactor and the vessel was sealed. The headspace was pressurized with hydrogen gas (80 psi) and vented. Hydrogen was again added and frequently refilled to maintain the pressure between ca. 60-80 psi. When the reaction no longer consumed hydrogen (ca. 10 refills over ca. 16-24 h), the pressure was released, the mixture was filtered (Celite), and the filtrate was concentrated to afford ca. a 1.0:0.30 mixture of 4-carboethoxyalerolactone (CEtVL) and 5ethoxy-2-methyl-5-oxopentanoic acid (S2). This mixture was diluted in EtOAc and washed with $\mathrm{NaHCO}_{3}(3 \mathrm{x})$. The aqueous bicarbonate layers were combined, saturated with $\mathrm{NaCl}$, and extracted with EtOAc (1x). The combined organic layers were washed with brine, dried $\left(\mathrm{MgSO}_{4}\right)$, and concentrated to afford 4-carboethoxyvalerolactone (CEtVL, $25.1 \mathrm{~g}, 146 \mathrm{mmol}$, $61 \%$ yield) as a colorless oil.

Typically, the lactone was stirred for ca. $1 \mathrm{~h}$ over $\mathrm{CaH}_{2}$ at $50{ }^{\circ} \mathrm{C}$ under vacuum $(0.05 \mathrm{mmHg}-0.1$ $\mathrm{mmHg}$ ) and distilled in vacuo. This distillation over $\mathrm{CaH}_{2}$ was repeated two to three times prior to polymerization. In each distillation, the first 5-10\% of the distillate was discarded. In the last distillation, CEtVL was distilled directly into a flame-dried scintillation vial and backfilled with $\mathrm{N}_{2}$ prior to being capped with a Teflon-lined capped and placed into a glovebox.

A portion of the aqueous $\mathrm{NaHCO}_{3}$ layer above was acidified with $3 \mathrm{M} \mathrm{HCl}$ and extracted with EtOAc (3x). The combined organic layers were washed with brine, dried $\left(\mathrm{MgSO}_{4}\right)$, and concentrated to give a colorless oil containing, predominantly, the 5-ethoxy-4-methyl-5oxopentanoic acid (S2). A portion was further purified via MPLC (silica gel 7:3 Hex: EtOAc).

\section{Data for CEtVL}

${ }^{1} \mathbf{H}$ NMR $\left(500 \mathrm{MHz}, \mathrm{CDCl}_{3}\right) \delta 4.50\left(\mathrm{ddd}, J=11.4,5.3,0.9 \mathrm{~Hz}, 1 \mathrm{H},(\mathrm{CO}) \mathrm{O}-\mathrm{CH}_{e q} \mathrm{H}_{\mathrm{ax}} \mathrm{CH}\right), 4.48$ $\left(\mathrm{dd}, J=11.6,7.5 \mathrm{~Hz}, 1 \mathrm{H},(\mathrm{CO}) \mathrm{O}-\mathrm{CH}_{\mathrm{eq}} H_{a x} \mathrm{CH}\right), 4.20^{+}\left(\mathrm{dq}, J=11.1,7.0 \mathrm{~Hz}, 1 \mathrm{H},-\mathrm{CO}_{2} \mathrm{CH}_{a} \mathrm{H}_{\mathrm{b}} \mathrm{CH}_{3}\right.$ ), 4.20- (dq, $J=11.1,7.0 \mathrm{~Hz}, 1 \mathrm{H},-\mathrm{CO}_{2} \mathrm{CH}_{\mathrm{a}} H_{b} \mathrm{CH}_{3}$ ), 2.92 (dddd, $J=7.7,7.7,6.6,5.3 \mathrm{~Hz}, 1 \mathrm{H}, \mathrm{CH}$ $\left.\mathrm{CO}_{2} \mathrm{Et}\right), 2.69\left(\mathrm{ddd}, J=17.5,6.9,6.9 \mathrm{~Hz}, 1 \mathrm{H},-\mathrm{CH}_{a} \mathrm{H}_{\mathrm{b}}(\mathrm{CO}) \mathrm{OCH}_{2}\right), 2.56(\mathrm{ddd}, J=17.5,7.5,7.5 \mathrm{~Hz}$, $1 \mathrm{H},-\mathrm{CH}_{\mathrm{a}} \mathrm{H}_{b}(\mathrm{CO}) \mathrm{OCH}_{2}$ ), 2.22 (dddd, $1 \mathrm{H}, J=13.9,7.5,7.5,7.5 \mathrm{~Hz},-\mathrm{O}_{2} \mathrm{CCH}_{2} \mathrm{H}_{\mathrm{eq}} C H_{a x} \mathrm{CH}-$ ), 2.19 (dddd, $J=14.0,7.6,7.6,6.7,0.9 \mathrm{~Hz}, 1 \mathrm{H},-\mathrm{O}_{2} \mathrm{CCH}_{2} \mathrm{H}_{e a} \mathrm{CH}_{\mathrm{ax}} \mathrm{CH}$ ), and $1.29(\mathrm{t}, J=7.0 \mathrm{~Hz}, 3 \mathrm{H}$, $\mathrm{CO}_{2} \mathrm{CH}_{2} \mathrm{CH}_{3}$ ).

${ }^{13} \mathbf{C}$ NMR $\left(125 \mathrm{MHz}, \mathrm{CDCl}_{3}\right) \delta 171.5,170.5,68.9,61.6,38.3,28.3,21.8$, and 14.3.

IR (neat, selected peaks): 2982, 2908, 1723 (strong), 1459, 1371, and $1241 \mathrm{~cm}^{-1}$.

bp: $111-114{ }^{\circ} \mathrm{C} @ 0.1 \mathrm{mmHg}$ and $96-105^{\circ} \mathrm{C} @ 0.05 \mathrm{mmHg}$. 
HRMS: Calculated for $\left(\mathrm{C}_{8} \mathrm{H}_{12} \mathrm{O}_{4} \mathrm{Na}\right)^{+}$195.0628; found: 195.0626

\section{Data for 5-Ethoxy-4-methyl-5-oxopentanoic acid (S2) ${ }^{7}$}

${ }^{1} \mathbf{H}$ NMR $\left(500 \mathrm{MHz}, \mathrm{CDCl}_{3}\right) \delta 11.32\left(\right.$ br s, $\left.1 \mathrm{H},-\mathrm{CO}_{2} \mathrm{H}\right), 4.14\left(\mathrm{q}, J=7.1 \mathrm{~Hz}, 2 \mathrm{H}, \mathrm{CO}_{2} \mathrm{CH}_{2} \mathrm{CH}_{3}\right)$, $2.50\left(\mathrm{dqd}, J=8.4,7.0,5.8 \mathrm{~Hz}, 1 \mathrm{H}, \mathrm{CHCO}_{2} \mathrm{Et}\right), 2.42(\mathrm{ddd}, J=16.6,8.6,6.5 \mathrm{~Hz}, 1 \mathrm{H}$, $\mathrm{HO}_{2} \mathrm{CCH}_{a} \mathrm{H}_{\mathrm{b}}$ ), 2.39 (ddd, $J=16.6,8.3,7.0 \mathrm{~Hz}, 1 \mathrm{H}, \mathrm{HO}_{2} \mathrm{CCH}_{\mathrm{a}} H_{b}$ ), 1.98 (dddd, $J=13.8,8.3,8.3$, $6.6 \mathrm{~Hz}, 1 \mathrm{H}, \mathrm{CH}_{c} \mathrm{H}_{\mathrm{d}} \mathrm{CHCO}_{2} \mathrm{Et}$ ), 1.79 (dddd, $J=13.9,8.7,7.0,5.9 \mathrm{~Hz}, \mathrm{CH}_{\mathrm{c}} H_{d} \mathrm{CHCO}_{2} \mathrm{Et}$ ), and 1.26 (t, $J=7.1 \mathrm{~Hz}, 3 \mathrm{H}, \mathrm{CH}_{2} \mathrm{CH}_{3}$, and 1.19 (d, $J=7.0 \mathrm{~Hz}, 3 \mathrm{H}, \mathrm{CH}_{3} \mathrm{CHCO}_{2} \mathrm{Et}$ ).

${ }^{13} \mathrm{C}$ NMR $\left(125 \mathrm{MHz}, \mathrm{CDCl}_{3}\right) \delta 179.0,176.0,60.6,38.8,31.7,28.4,17.2$, and 14.4.

IR (neat, selected peaks): 3450-2989 (br), 2974, 2938, 1729 (strong), and 1709 (strong) $\mathrm{cm}^{-1}$. HRMS Calculated for $\left(\mathrm{C}_{8} \mathrm{H}_{15} \mathrm{O}_{4}\right)^{+}$175.0965; found: 175.0961 . 


\section{4-Carboisopropoxyvalerolactone (iso-propyl 6-oxotetrahydro-2H-pyran-3-carboxylate, CiPrVL) and 5-iso-Propoxy-4-methyl-5-oxopentanoic acid (S3)}<smiles>CCCOC(=O)c1ccc(=O)oc1</smiles>

2-iPr

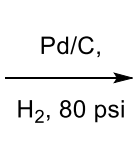

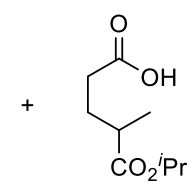

S3

Isopropyl coumalate (2-iPr, $45.0 \mathrm{~g}, 247 \mathrm{mmol}$ ) and $\mathrm{Pd} / \mathrm{C}[3.0 \mathrm{~g}, 5 \% \mathrm{Pd} / \mathrm{C}]$ were placed into a 500 -mL Fisher-Porter pressure vessel ${ }^{8}$. THF $(250 \mathrm{~mL})$ was added by being poured down the side of the reactor and the vessel was sealed. The headspace was pressurized with hydrogen gas (80 psi) and vented. Hydrogen was again added and the mixture was stirred at ambient temperature. As the pressure fell, the headspace was refilled to ca. 60-80 psi. When the reaction no longer consumed hydrogen (ca. 10 refills over ca. 16-24 h), the final pressure was released, the mixture was filtered (Celite), and the filtrate was concentrated to afford ca. a 1.0:0.63 mixture of 4-carboisopropoxyalerolactone (CiPrVL) and 5-isopropoxy-2-methyl-5oxopentanoic acid (S3). The mixture was diluted in EtOAc and washed with $\mathrm{NaHCO}_{3}(3 \mathrm{x})$. The aqueous bicarbonate layers were combined, saturated with solid $\mathrm{NaCl}$, and extracted with EtOAc (1x). The combined organic layers were washed with brine, dried $\left(\mathrm{MgSO}_{4}\right)$, and concentrated to afford 4-carboisopropoxyvalerolactone (CiPrVL, $26.5 \mathrm{~g}, 142 \mathrm{mmol}, 58 \%$ yield) as a colorless oil.

CiPrVL was stirred for ca. $1 \mathrm{~h}$ over $\mathrm{CaH}_{2}$ at $50^{\circ} \mathrm{C}$ under vacuum $(0.05 \mathrm{mmHg}-0.1 \mathrm{mmHg})$ and distilled from $\mathrm{CaH}_{2}$ in vacuo (two or three times) prior to polymerization. In each distillation, the first $5-10 \%$ of the distillate was discarded.

A portion of the aqueous $\mathrm{NaHCO}_{3}$ layer above was acidified with $3 \mathrm{M} \mathrm{HCl}$ and extracted with EtOAc (3x). The combine organic layers were washed with brine, dried $\left(\mathrm{MgSO}_{4}\right)$, and concentrated to give a colorless oil containing, predominantly, 5-isopropoxy-4-methyl-5oxopentanoic acid (S3). A portion was further purified via MPLC (silica gel 7:3 Hex: EtOAc).

\section{Data for CiPrVL}

${ }^{1} \mathbf{H}$ NMR $\left(500 \mathrm{MHz}, \mathrm{CDCl}_{3}\right) \delta 5.06$ (septet, $\left.J=6.3 \mathrm{~Hz}, 1 \mathrm{H},-\mathrm{CO}_{2} \mathrm{CH}\left(\mathrm{CH}_{3}\right)_{2}\right), 4.49$ (ddd, $J=11.5$, $\left.5.3,1.0 \mathrm{~Hz}, 1 \mathrm{H},(\mathrm{CO}) \mathrm{O}-\mathrm{CH}_{e q} \mathrm{H}_{\mathrm{ax}} \mathrm{CH}\right), 4.47\left(\mathrm{dd}, J=11.5,7.6 \mathrm{~Hz}, 1 \mathrm{H},(\mathrm{CO}) \mathrm{O}-\mathrm{CH}_{\mathrm{eq}} H_{a x} \mathrm{CH}\right), 2.88$ (dddd, $J=7.7,7.7,6.6,5.3 \mathrm{~Hz}, 1 \mathrm{H}, \mathrm{CH}-\mathrm{CO}_{2}{ }^{i} \mathrm{Pr}$ ), 2.68 (ddd, $J=17.5,6.9,6.9 \mathrm{~Hz}, 1 \mathrm{H}$, $\left.\mathrm{CH}_{a} \mathrm{H}_{\mathrm{b}}(\mathrm{CO}) \mathrm{OCH}_{2}\right), 2.56\left(\mathrm{ddd}, J=17.5,7.5,7.5 \mathrm{~Hz}, 1 \mathrm{H},-\mathrm{CH}_{\mathrm{a}} H_{b}(\mathrm{CO}) \mathrm{OCH}_{2}\right), 2.21(\mathrm{dddd}, 1 \mathrm{H}, J=$ $13.9,7.5,7.5,7.5 \mathrm{~Hz},-\mathrm{O}_{2} \mathrm{CCH}_{2} \mathrm{H}_{\mathrm{eq}} \mathrm{CH}_{a x} \mathrm{CH}-$ ), 2.19 (dddd, $J=14.0,7.6,7.6,6.7,1.0 \mathrm{~Hz}, 1 \mathrm{H}$, $\left.\mathrm{O}_{2} \mathrm{CCH}_{2} \mathrm{H}_{e a} \mathrm{CH}_{\mathrm{ax}} \mathrm{CH}\right)$, and $1.26\left(\mathrm{~d}, \mathrm{~J}=6.3 \mathrm{~Hz}, 6 \mathrm{H}, \mathrm{CO}_{2} \mathrm{CH}\left(\mathrm{CH}_{3}\right)_{2}\right)$.

${ }^{13} \mathrm{C}$ NMR $\left(125 \mathrm{MHz}, \mathrm{CDCl}_{3}\right) \delta 170.9,170.6,69.2,69.0,38.4,28.3,21.9,21.84$, and 21.78.

IR (neat, selected peaks): 2983, 2938, 1729 (strong) (with a shoulder), 1458, 1374, and $1171 \mathrm{~cm}^{-}$ 1 .

HRMS: Calculated for $\left(\mathrm{C}_{9} \mathrm{H}_{14} \mathrm{O}_{4} \mathrm{Na}\right)^{+}$209.0784; found: 209.0781.

bp: $117-120{ }^{\circ} \mathrm{C} @ 0.05 \mathrm{mmHg}$.

Data for 5-isoPropoxy-4-methyl-5-oxopentanoic acid (S3)

${ }^{1} \mathbf{H}$ NMR $\left(500 \mathrm{MHz}, \mathrm{CDCl}_{3}\right) \delta 10.99$ (br s, $1 \mathrm{H},-\mathrm{CO}_{2} H$ ), 5.01 [septet, $J=6.3 \mathrm{~Hz}, 1 \mathrm{H}$, $\mathrm{CO}_{2} \mathrm{CH}\left(\mathrm{CH}_{3}\right)_{2}$ ], $2.46\left(\mathrm{dqd}, J=8.4,7.0,5.8 \mathrm{~Hz}, 1 \mathrm{H}, \mathrm{CHCO}_{2}{ }^{\mathrm{i}} \mathrm{Pr}\right), 2.42$ (ddd, $J=16.4,8.6,6.5 \mathrm{~Hz}$, 
$\left.1 \mathrm{H}, \mathrm{HO}_{2} \mathrm{CCH}_{a} \mathrm{H}_{\mathrm{b}}\right), 2.38\left(\mathrm{ddd}, J=16.5,8.4,7.0 \mathrm{~Hz}, 1 \mathrm{H}, \mathrm{HO}_{2} \mathrm{CCH}_{\mathrm{a}} H_{b}\right), 1.96$ (dddd, $J=13.9,8.4$, 8.4, $6.6 \mathrm{~Hz}, 1 \mathrm{H}, \mathrm{CH}_{c} \mathrm{H}_{\mathrm{d}} \mathrm{CHCO}_{2}{ }^{\mathrm{i}} \mathrm{Pr}$ ), 1.77 (dddd, $J=13.9,8.7,7.0,5.9 \mathrm{~Hz}, \mathrm{CH}_{\mathrm{c}} H_{d} \mathrm{CHCO}_{2}{ }^{\mathrm{i}} \mathrm{Pr}$ ), $1.24\left[\mathrm{~d}, J=6.3 \mathrm{~Hz}, 3 \mathrm{H}, \mathrm{CH}\left(\mathrm{CH}_{3}\right)_{2}\right], 1.23\left[\mathrm{~d}, J=6.3 \mathrm{~Hz}, 3 \mathrm{H}, \mathrm{CH}\left(\mathrm{CH}_{3}\right)_{2}\right]$, and $1.17(\mathrm{~d}, J=7.0 \mathrm{~Hz}$, $\left.3 \mathrm{H}, \mathrm{CH}_{3} \mathrm{CHCO}_{2}{ }^{\mathrm{i}} \mathrm{Pr}\right)$.

${ }^{13} \mathrm{C}$ NMR $\left(125 \mathrm{MHz}, \mathrm{CDCl}_{3}\right) \delta$ 179.3, 175.6, 67.9, 39.0, 31.7, 28.3, 22.0, 21.9, and 17.2.

IR (neat, selected peaks): 3124 (br), 2979, 2934, 1723 (strong), 1707 (strong), 1445, 1415, 1167, $1105 \mathrm{~cm}^{-1}$.

HRMS Calculated for $\left(\mathrm{C}_{9} \mathrm{H}_{17} \mathrm{O}_{4}\right)^{+} 189.1121$; found: 189.1117 . 
Butyl 6-oxotetrahydro-2H-pyran-3-carboxylate (CnBuVL) and 2-Methyl-5-pentanedioic acid, 1-butyl ester (S4)

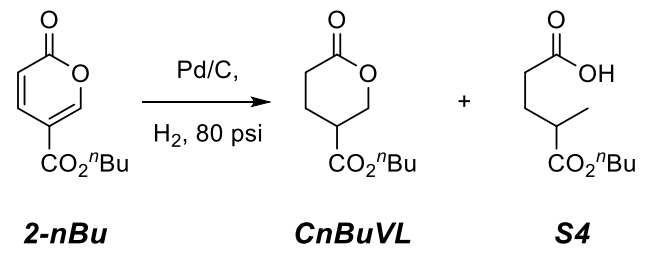

n-Butyl coumalate (2-nBu,34.06 g, $173.7 \mathrm{mmol}$ ) and $\mathrm{Pd} / \mathrm{C}[2.0 \mathrm{~g}, 5 \% \mathrm{Pd} / \mathrm{C}$ (wt. Pd/C/wt. coumalate)] were placed into a 500-mL Fisher-Porter pressure vessel ${ }^{8}$. THF $(200 \mathrm{~mL})$ was added by being poured carefully down the side of the reactor and the vessel was then sealed. The headspace was pressurized with hydrogen gas ( $80 \mathrm{psi})$ and vented. Hydrogen was again added and the mixture was stirred at ambient temperature. As the pressure fell, the headspace was refilled to ca. 60-80 psi. When the reaction no longer consumed hydrogen (ca. 8 refills over ca. 16-24 h), the final overpressure was released, the mixture was filtered (Celite), and the filtrate was concentrated to afford ca. a 1.0:0.67 mixture of 4-carbo- $n$-butoxyvalerolactone (CnBuVL) and 5-n-butoxy-2-methyl-5-oxopentanoic acid (S4). The mixture was diluted in EtOAc and washed with $\mathrm{NaHCO}_{3}(3 \mathrm{x})$. The combined organic layers were washed with brine, dried $\left(\mathrm{MgSO}_{4}\right)$, and concentrated. The mixture was distilled in vacuo over calcium hydride to afford 4-carbo- $n$-butoxyvalerolactone (CnBuVL, $19.86 \mathrm{~g}, 57 \%$ yield) as a colorless oil. CnBuVL was distilled in vacuo two more times from $\mathrm{CaH}_{2}$ prior to polymerization. In each distillation, the first $5-10 \%$ of the distillate was discarded.

A portion of the aqueous $\mathrm{NaHCO}_{3}$ layer from above was acidified with $3 \mathrm{M} \mathrm{HCl}$ and extracted with EtOAc (3x). The combine organic layers were washed with brine, dried $\left(\mathrm{MgSO}_{4}\right)$, and concentrated to give a colorless oil containing, predominantly, the 5- $n$-butoxy-4-methyl-5oxopentanoic acid (S4). A portion was further purified via MPLC (silica gel 7:3 Hex: EtOAc).

\section{Data for CnBuVL}

${ }^{1} \mathbf{H}$ NMR $\left(500 \mathrm{MHz}, \mathrm{CDCl}_{3}\right) \delta 4.50\left(\mathrm{ddd}, J=11.5,5.3,0.9 \mathrm{~Hz}, 1 \mathrm{H},(\mathrm{CO}) \mathrm{O}-\mathrm{CH}_{e q} \mathrm{H}_{\mathrm{ax}} \mathrm{CH}\right), 4.48$ $\left(\mathrm{dd}, J=11.5,7.6 \mathrm{~Hz}, 1 \mathrm{H},(\mathrm{CO}) \mathrm{O}-\mathrm{CH}_{\mathrm{eq}} H_{a x} \mathrm{CH}\right), 4.15\left(\mathrm{t}, J=6.7 \mathrm{~Hz}, 2 \mathrm{H},-\mathrm{CO}_{2} \mathrm{CH}_{2} \mathrm{CH}_{2}-\right), 2.92$ (dddd, $J=7.7,7.7,6.6,5.3 \mathrm{~Hz}, 1 \mathrm{H}, \mathrm{CH}-\mathrm{CO}_{2}{ }^{n} \mathrm{Bu}$ ), 2.69 (ddd, $J=17.6,6.9,6.9 \mathrm{~Hz}, 1 \mathrm{H}$, $\left.\mathrm{CH}_{a} \mathrm{H}_{\mathrm{b}}(\mathrm{CO}) \mathrm{OCH}_{2}\right), 2.56\left(\mathrm{ddd}, J=17.6,7.5,7.5 \mathrm{~Hz}, 1 \mathrm{H},-\mathrm{CH}_{\mathrm{a}} H_{b}(\mathrm{CO}) \mathrm{OCH}_{2}\right), 2.22(\mathrm{dddd}, 1 \mathrm{H}, J=$ 13.9, 7.6, 7.6, 7.6 Hz, $-\mathrm{O}_{2} \mathrm{CCH}_{2} \mathrm{H}_{\mathrm{eq}} \mathrm{CH}_{a x} \mathrm{CH}-$ ), 2.19 (dddd, $J=14.0,7.5,7.5,6.7,0.9 \mathrm{~Hz}, 1 \mathrm{H}$, $\mathrm{O}_{2} \mathrm{CCH}_{2} \mathrm{H}_{e a} \mathrm{CH}_{\mathrm{ax}} \mathrm{CH}$ ), 1.66-1.61 (m, 2H, $-\mathrm{CH}_{2} \mathrm{CH}_{2} \mathrm{CH}_{2} \mathrm{CH}_{3}$ ), 1.39 (br sext, $J=7.5 \mathrm{~Hz}, 2 \mathrm{H}$, $\left.\mathrm{CH}_{2} \mathrm{CH}_{2} \mathrm{CH}_{2} \mathrm{CH}_{3}\right)$, and $0.94\left(\mathrm{t}, \mathrm{J}=7.4 \mathrm{~Hz}, 3 \mathrm{H}, \mathrm{CO}_{2}\left(\mathrm{CH}_{2}\right)_{2} \mathrm{CH}_{3}\right)$.

${ }^{13} \mathrm{C}$ NMR $\left(125 \mathrm{MHz}, \mathrm{CDCl}_{3}\right) \delta 171.5,170.5,68.9,65.5,38.4,30.7,28.3,21.8,19.2$, and 13.8.

IR (neat, selected peaks): 2960, 2935, 2878, 1726 (strong), 1459, 1172, and $1054 \mathrm{~cm}^{-1}$.

HRMS: Calculated for $\left(\mathrm{C}_{10} \mathrm{H}_{16} \mathrm{O}_{4} \mathrm{Na}\right)^{+} 223.0941$; found: 223.0932.

bp: $140-143{ }^{\circ} \mathrm{C} @ 0.1 \mathrm{mmHg}$. 


\section{Data for 5-n-Butoxy-4-methyl-5-oxopentanoic acid (S4)}

${ }^{1} \mathbf{H}$ NMR $\left(500 \mathrm{MHz}, \mathrm{CDCl}_{3}\right) \delta 10.6$ (br s, $\left.1 \mathrm{H},-\mathrm{CO}_{2} \mathrm{H}\right), 4.08\left(\mathrm{t}, J=6.7 \mathrm{~Hz}, 2 \mathrm{H}, \mathrm{CO}_{2} \mathrm{CH}_{2}\right), 2.51$ (dqd, $J=8.4,7.0,5.7 \mathrm{~Hz}, 1 \mathrm{H}, \mathrm{CHCO}_{2}{ }^{n} \mathrm{Bu}$ ), 2.42 (ddd, $J=16.6,8.7,6.5 \mathrm{~Hz}, 1 \mathrm{H}, \mathrm{HO}_{2} \mathrm{CCH}_{a} \mathrm{H}_{\mathrm{b}}$ ), 2.39 (ddd, $J=16.5,8.3,6.9 \mathrm{~Hz}, 1 \mathrm{H}, \mathrm{HO}_{2} \mathrm{CCH}_{\mathrm{a}} H_{b}$ ), 1.98 (dddd, $J=13.9,8.3,8.3,6.6 \mathrm{~Hz}, 1 \mathrm{H}$, $\mathrm{CH}_{c} \mathrm{H}_{\mathrm{d}} \mathrm{CHCO}_{2}{ }^{n} \mathrm{Bu}$ ), 1.83-1.75 (nfom, 2H, $\mathrm{CH}_{\mathrm{c}} \mathrm{H}_{d} \mathrm{CHCO}_{2}{ }^{n} \mathrm{Bu}$ ), 1.64-1.58 (m, 2H, -

$\mathrm{CH}_{2} \mathrm{CH}_{2} \mathrm{CH}_{2} \mathrm{CH}_{3}$ ), 1.38 (qt, $J=7.5,7.5 \mathrm{~Hz}, 2 \mathrm{H},-\mathrm{CH}_{2} \mathrm{CH}_{2} \mathrm{CH}_{2} \mathrm{CH}_{3}$ ), and 1.19 (d, $J=7.0 \mathrm{~Hz}, 3 \mathrm{H}$, $\left.\mathrm{CH}_{3} \mathrm{CHCO}_{2}{ }^{n} \mathrm{Bu}\right)$, and $0.94\left(\mathrm{t}, J=7.4 \mathrm{~Hz}, 3 \mathrm{H}, \mathrm{CH}_{2} \mathrm{CH}_{3}\right)$.

${ }^{13} \mathrm{C}$ NMR $\left(125 \mathrm{MHz}, \mathrm{CDCl}_{3}\right) \delta 178.6,176.1,64.5,38.9,31.6,30.8,28.4,19.3,17.3$, and 13.9.

IR (neat, selected peaks): 3450-2989 (br), 2963, 2939, 1732 (strong), and 1715 (strong) cm ${ }^{-1}$.

HRMS Calculated for $\left(\mathrm{C}_{10} \mathrm{H}_{19} \mathrm{O}_{4}\right)^{+} 203.1278$; found: 203.1274 . 


\section{4-Carbo-t-butoxyvalerolactone (tert-Butyl 6-oxotetrahydro-2H-pyran-3-carboxylate, (C'BuVL) and 5-t-Butoxy-4-methyl-5-oxopentanoic acid (S5)}

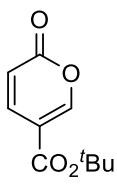

2-tBu

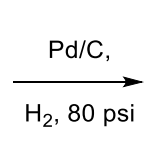

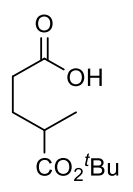

S5

$t$-Butyl coumalate (2-tBu, $1.02 \mathrm{~g}, 5.20 \mathrm{mmol}), \mathrm{Pd} / \mathrm{C}(100 \mathrm{mg})$, and a stir bar were added to 6dram vial. EtOAc $(10 \mathrm{~mL})$ was added by being poured carefully down the side of the vial. The vial was placed directly into a 150 -mL Fisher-Porter pressure vessel ${ }^{8}$ and the apparatus was sealed. The headspace was pressurized with hydrogen gas (80 psi) and vented. Hydrogen was again added and the mixture was stirred at ambient temperature. When the reaction no longer consumed hydrogen, the final overpressure was released, the mixture was filtered (Celite), and the filtrate was concentrated to afford ca. a 1.0:0.52 mixture of 4-carbo-t-butoxyvalerolactone (CtBuVL) and 5-t-butoxy-2-methyl-5-oxopentanoic acid (S5). The mixture was diluted in EtOAc and washed with $\mathrm{NaHCO}_{3}(3 \mathrm{x})$. The combined organic layers were washed with brine, dried $\left(\mathrm{MgSO}_{4}\right)$, and concentrated to afford 4-carbo-t-butoxyvalerolactone (CtBuVL, $692 \mathrm{mg}$, 67\% yield) as a colorless oil. CtBuVL was further dried and purified via MPLC (silica, 3:1 EtOAc:Hex) prior to polymerization. Additionally, the entire aqueous layers from extraction were combined, acidified $(1 \mathrm{M} \mathrm{HCl})$, dried $\left(\mathrm{MgSO}_{4}\right)$, concentrated, and purified via MPLC (silica, 7:3 Hex:EtOAc) to afford 5-t-butoxy-4-methyl-5-oxopentanoic acid (S5, $265 \mathrm{mg}, 26 \%$ yield) as a colorless oil.

\section{Data for CtBuVL}

${ }^{1} \mathbf{H}$ NMR $\left(500 \mathrm{MHz}, \mathrm{CDCl}_{3}\right) \delta 4.46\left(\mathrm{ddd}, J=11.5,5.2,0.9 \mathrm{~Hz}, 1 \mathrm{H},(\mathrm{CO}) \mathrm{O}-\mathrm{CH}_{e q} \mathrm{H}_{\mathrm{ax}} \mathrm{CH}\right), 4.43$ $\left(\mathrm{dd}, J=11.5,7.8, \mathrm{~Hz}, 1 \mathrm{H},(\mathrm{CO}) \mathrm{O}-\mathrm{CH}_{\mathrm{eq}} H_{a x} \mathrm{CH}\right.$ ), 2.82 (dddd, $J=7.8,7.8,6.6,5.2 \mathrm{~Hz}, 1 \mathrm{H}, \mathrm{CH}$ $\mathrm{CO}_{2}{ }^{t} \mathrm{Bu}$ ), 2.67 (ddd, $\left.J=17.5,6.9,6.9 \mathrm{~Hz}, 1 \mathrm{H},-\mathrm{CH}_{a} \mathrm{H}_{\mathrm{b}}(\mathrm{CO}) \mathrm{OCH}_{2}\right), 2.54$ (ddd, $J=17.5,7.5,7.5$ $\left.\mathrm{Hz}, 1 \mathrm{H},-\mathrm{CH}_{\mathrm{a}} \mathrm{H}_{b}(\mathrm{CO}) \mathrm{OCH}_{2}\right), 2.22$ (ddd, $1 \mathrm{H}, J=13.9,7.6,7.6,7.6 \mathrm{~Hz},-\mathrm{O}_{2} \mathrm{CCH}_{2} \mathrm{H}_{\mathrm{a}} \mathrm{CH}$ CH-), 2.19 (dddd, $\left.J=14.0,7.5,7.5,6.6,1.0 \mathrm{~Hz}, 1 \mathrm{H},-\mathrm{O}_{2} \mathrm{CCH}_{2} \mathrm{H}_{a} \mathrm{CH}_{\mathrm{b}} \mathrm{CH}\right)$, and $1.47\left(\mathrm{~s}, 9 \mathrm{H}, \mathrm{CO}_{2}{ }^{t} B u\right)$.

${ }^{13} \mathbf{C ~ N M R}\left(125 \mathrm{MHz}, \mathrm{CDCl}_{3}\right) \delta 170.8,170.6,82.2,69.2,39.1,28.3,28.1$, and 21.8.

IR (neat, selected peaks): 2977, 2934, 1721 (strong), 1477, 1393, 1148, $1055 \mathrm{~cm}^{-1}$.

HRMS: Calculated for $\left(\mathrm{C}_{10} \mathrm{H}_{16} \mathrm{O}_{4} \mathrm{Na}\right)^{+} 223.0941$; found: 223.0954 .

\section{Data for 5-tButoxy-4-methyl-5-oxopentanoic acid (S5)}

${ }^{1} \mathbf{H} \mathbf{N M R}\left(500 \mathrm{MHz}, \mathrm{CDCl}_{3}\right) \delta 10.4\left(\mathrm{br} \mathrm{s}, 1 \mathrm{H},-\mathrm{CO}_{2} H\right), 2.45-2.33\left(\mathrm{~m}, 3 \mathrm{H}, \mathrm{CHCO}_{2} t \mathrm{Bu}\right.$ and $\mathrm{CH}_{2} \mathrm{CO}_{2} \mathrm{H}$ ), 1.93 (dddd, $J=13.9,8.4,8.4,6.4 \mathrm{~Hz}, 1 \mathrm{H}, \mathrm{CH}_{c} \mathrm{H}_{\mathrm{d}} \mathrm{CHCO}_{2}{ }^{t} \mathrm{Bu}$ ), 1.74 (dddd, $J=14.0$, 8.7, 7.0, $\left.5.9 \mathrm{~Hz}, 1 \mathrm{H}, \mathrm{CH}_{\mathrm{c}} \mathrm{H}_{d} \mathrm{CHCO}_{2}{ }^{t} \mathrm{Bu}\right)$, and $1.45\left(\mathrm{~s}, 9 \mathrm{H},{ }^{t} \mathrm{Bu}\right)$.

${ }^{13}$ C NMR $\left(125 \mathrm{MHz}, \mathrm{CDCl}_{3}\right) \delta 179.3,175.4,80.5,39.7,31.8,28.5,28.2$, and 17.3.

IR (neat, selected peaks): 3450-2989 (br), 2976, 2937, 1723 (strong), and 1709 (strong) cm ${ }^{-1}$.

HRMS: Calculated for $\left(\mathrm{C}_{10} \mathrm{H}_{18} \mathrm{O}_{4} \mathrm{Na}\right)^{+} 225.1097$; found: 225.1088 . 


\section{2-(Bromomethyl)glutaric acid (4)}

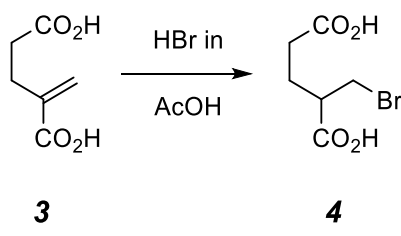

2-Methyleneglutaric acid (3) was prepared as previously reported. ${ }^{3,9}$ This acid (10.00 g, 69.4 $\mathrm{mmol}), \mathrm{HBr}$ in $\mathrm{AcOH}(48 \% \mathrm{w} / \mathrm{v}, 51 \mathrm{~mL}, 210 \mathrm{mmol})$, and a stir bar were added to an $8 \mathrm{oz}$. glass jar and sealed with a Teflon-lined cap. The contents were stirred for $20 \mathrm{~h}$ at ambient temperature. The reaction mixture was slowly poured into $600 \mathrm{~mL}$ of stirred $\mathrm{DI} \mathrm{H}_{2} \mathrm{O}$, and the aqueous suspension was extracted with EtOAc (3x ca. $400 \mathrm{~mL})$. The organic layers were combined, washed with $\mathrm{H}_{2} \mathrm{O}$ (ca. $200 \mathrm{~mL}$ ) and brine (ca. $400 \mathrm{~mL}$ ), dried over $\mathrm{MgSO}_{4}$, and concentrated. Toluene (ca. $150 \mathrm{~mL}$ ) was added to the light orange liquid to assist in the removal of acetic acid and placed under high vacuum to afford 2-(bromomethyl)glutaric acid (4, $13.64 \mathrm{~g}, 87 \%$ yield) as a white amorphous solid. This was stored in a freezer $\left(\mathrm{ca},-30^{\circ} \mathrm{C}\right)$ and used without further purification.

${ }^{1} \mathbf{H}$ NMR $\left(500 \mathrm{MHz}, \mathrm{DMSO}-d_{6}\right) \delta$ 12.75-11.93 (br s, 2H, $\left.\mathrm{CO}_{2} H\right), 3.67$ (dd, $J=10.0,4.6 \mathrm{~Hz}$, $\left.\mathrm{BrCH}_{a} \mathrm{H}_{\mathrm{b}}\right), 3.60\left(\mathrm{dd}, J=10.0,7.3 \mathrm{~Hz}, \mathrm{BrCH}_{\mathrm{a}} H_{b}\right), 2.77(\mathrm{dddd}, J=8.0,7.3,6.1,4.6 \mathrm{~Hz}, 1 \mathrm{H}$, $\mathrm{BrCH}_{2} \mathrm{CH}$ ), 2.29 (ddd, $J=16.6,8.6,6.7 \mathrm{~Hz}, 1 \mathrm{H}, \mathrm{CH}_{c} \mathrm{H}_{\mathrm{d}} \mathrm{CO}_{2} \mathrm{H}$ ), 2.26 (ddd, $J=16.6,8.2,7.1 \mathrm{~Hz}$, $1 \mathrm{H}, \mathrm{CH}_{\mathrm{c}} \mathrm{H}_{d} \mathrm{CO}_{2} \mathrm{H}$ ), 1.81 (dddd, $J=13.8,8.1,8.1,6.8 \mathrm{~Hz}, 1 \mathrm{H} \mathrm{CH}{ }_{a} \mathrm{H}_{\mathrm{b}} \mathrm{CH}_{2} \mathrm{CO}_{2} \mathrm{H}$ ), and 1.75 (dddd, $J$ $\left.=13.6,8.6,7.2,6.0 \mathrm{~Hz}, 1 \mathrm{H} \mathrm{CH} \mathrm{H}_{b} \mathrm{CH}_{2} \mathrm{CO}_{2} \mathrm{H}\right)$.

${ }^{13}$ C NMR (125 MHz, DMSO- $\left.d_{6}\right) \delta 173.8,173.6,46.1,34.3,30.9$, and 25.6.

IR (neat, selected peaks): 3340-2550 (br), 2923, 2854, 1688 (s), 1445, 1430, 1404, 1282, 1251, and $932 \mathrm{~cm}^{-1}$.

mp $103-109^{\circ} \mathrm{C}$. 


\section{4-Carbobenzoxyvalerolactone (benzyl 6-oxotetrahydro-2H-pyran-3-carboxylate, CBnVL)}

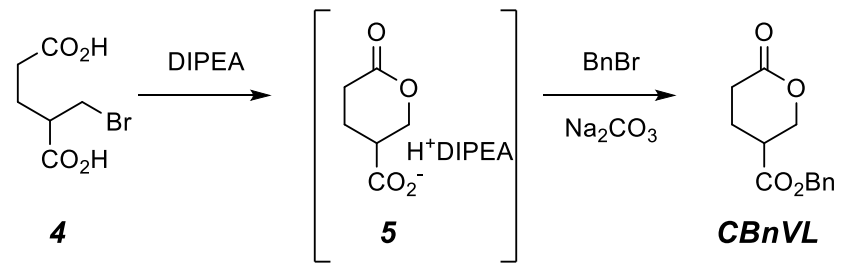

2-(Bromomethyl)glutaric acid (4, 1.0 equiv, $10.00 \mathrm{~g}, 44.46 \mathrm{mmol})$ and a stir bar were placed into a $500 \mathrm{~mL}$ round bottom flask. DCM $(170 \mathrm{~mL})$ was added followed by Hünig's base (3 equiv, $23.2 \mathrm{~mL}, 133.38 \mathrm{mmol}$ ) and the reaction mixture was stirred. After $16 \mathrm{~h}$, the reaction mixture was concentrated in vacuo to afford a white slurry. The slurry was resuspended in DMF (200 $\mathrm{mL}$ ) and benzyl bromide ( 1.5 equiv, $7.9 \mathrm{~mL}, 67 \mathrm{mmol}$ ) followed by $\mathrm{Na}_{2} \mathrm{CO}_{3}$ (3.0 equiv, $14.1 \mathrm{~g}$, $130 \mathrm{mmol}$ ) were added. The heterogeneous mixture was stirred overnight at room temperature. The mixture was concentrated in vacuo, and the white residue was partitioned into EtOAc (ca. $600 \mathrm{~mL}$ ) and water (ca. $100 \mathrm{~mL}$ ). The organic layer was washed with $\mathrm{NH}_{4} \mathrm{Cl}, \mathrm{NaHCO}_{3}$, and brine; dried with $\mathrm{MgSO}_{4}$, and concentrated to leave a yellow oil. The oil was further purified via column chromatography (silica gel, gradient elution solvent EtOAc:Hex 9:1, 3:1, 1:1) to afford 4-carbobenzoxyvalerolactone (CBnVL, $7.28 \mathrm{~g}, 70 \%$ yield) as a colorless, highly viscous oil.

${ }^{1} \mathbf{H}$ NMR $\left(500 \mathrm{MHz} \mathrm{CDCl}_{3}\right) \delta$ 7.41-7.32 (m, 5H, Ar), $5.19\left(\mathrm{~d}, J=12.2 \mathrm{~Hz}, 1 \mathrm{H}, \mathrm{ArCH}_{a} \mathrm{H}_{\mathrm{b}}\right), 5.16$ $\left(\mathrm{d}, J=12.2 \mathrm{~Hz}, 1 \mathrm{H}, \mathrm{ArCH}_{\mathrm{a}} H_{b}\right), 4.51$ (ddd, $\left.J=11.5,5.6,0.8 \mathrm{~Hz}, 1 \mathrm{H},(\mathrm{CO}) \mathrm{O}-\mathrm{CH}_{e q} \mathrm{H}_{\mathrm{ax}} \mathrm{CH}\right), 4.48$ $\left(\mathrm{dd}, J=11.6,7.2 \mathrm{~Hz}, 1 \mathrm{H},(\mathrm{CO}) \mathrm{O}-\mathrm{CH}_{\mathrm{eq}} H_{a x} \mathrm{CH}\right), 2.97$ (dddd, $J=7.7,7.7,6.7,5.6 \mathrm{~Hz}, 1 \mathrm{H}, \mathrm{CH}$ $\mathrm{CO}_{2} \mathrm{Bn}$ ), 2.67 (ddd, $\left.J=17.5,6.9,6.9 \mathrm{~Hz}, 1 \mathrm{H},-\mathrm{CH}_{a} \mathrm{H}_{\mathrm{b}}(\mathrm{CO}) \mathrm{OCH}_{2}\right), 2.55(\mathrm{ddd}, J=17.5,7.5,7.5$ $\mathrm{Hz}, 1 \mathrm{H},-\mathrm{CH}_{\mathrm{a}} \mathrm{H}_{b}(\mathrm{CO}) \mathrm{OCH}_{2}$ ), 2.23 (dddd, $1 \mathrm{H}, J=13.9,7.6,7.6,7.6 \mathrm{~Hz},-\mathrm{O}_{2} \mathrm{CCH}_{2} \mathrm{H}_{\mathrm{eq}} \mathrm{CH}_{a x} \mathrm{CH}-$ ), and 2.19 (dddd, $J=14.0,7.5,7.5,6.7,0.9 \mathrm{~Hz}, 1 \mathrm{H},-\mathrm{O}_{2} \mathrm{CCH}_{2} \mathrm{H}_{e a} \mathrm{CH}_{\mathrm{ax}} \mathrm{CH}$ ).

${ }^{13} \mathbf{C}$ NMR $\left(125 \mathrm{MHz}, \mathrm{CDCl}_{3}\right) \delta 171.2,170.4,135.3,128.85,128.75,128.4,68.8,67.3,38.3$, 28.2, and 21.8 .

IR (neat, selected peaks): 3065, 3032, 2958, 2913, 1727 (s), 1498, 1241, 1155, and $1054 \mathrm{~cm}^{-1}$.

HRMS: Calculated for $\left(\mathrm{C}_{13} \mathrm{H}_{14} \mathrm{O}_{4} \mathrm{Na}\right)^{+} 257.0784$; found: 257.0783.

bp: $194-199{ }^{\circ} \mathrm{C} @ 0.05 \mathrm{mmHg}$. Under these conditions, a small amount (ca. $5 \mathrm{~mol} \%$ ) of an elimination product is formed. Therefore, samples of this monomer used for the ROTEP were purified by MPLC. 


\section{4-Carbo(2-ethyl)hexoxyvalerolactone (2-ethylhexyl 6-oxotetrahydro-2H-pyran-3- carboxylate, $\mathrm{C}^{2}$ EtHexVL)}

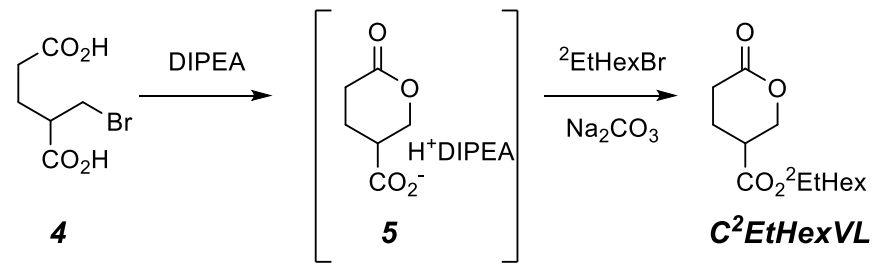

2-(Bromomethyl)glutaric acid (4, 1.0 equiv, $1.50 \mathrm{~g}, 6.67 \mathrm{mmol})$ and a stir bar were placed into a 100-mL culture tube. DCM (20 mL) was added followed by Hünig's base (3 equiv, $3.5 \mathrm{~mL}, 20$ mmol). The reaction mixture was stirred at ambient temperature for $20 \mathrm{~h}$ and concentrated in vacuo to afford a white slurry. The slurry was resuspended in DMF (20 mL) and 1-bromo-2ethylhexane ( 1.5 equiv, $1.8 \mathrm{~mL}, 10 \mathrm{mmol}$ ) followed by $\mathrm{Na}_{2} \mathrm{CO}_{3}$ (1.5 equiv, $\left.1.06 \mathrm{~g}, 10 \mathrm{mmol}\right)$ were added. The heterogeneous mixture was stirred at ambient temperature for $24 \mathrm{~h}, 35^{\circ} \mathrm{C}$ for 20 $\mathrm{h}$, and $55^{\circ} \mathrm{C}$ for $40 \mathrm{~h}$ to achieve full conversion. The mixture was concentrated in vacuo, and the white residue was partitioned into EtOAc (ca. $80 \mathrm{~mL}$ ) and water (ca. $30 \mathrm{~mL}$ ). The organic layer was washed with $\mathrm{NH}_{4} \mathrm{Cl}, \mathrm{NaHCO}_{3}$, and brine; dried with $\mathrm{MgSO}_{4}$; and concentrated in vacuo to leave a yellow oil. The oil was further purified via column chromatography (silica gel, 1:3 EtOAc:Hex) to afford 4-carbo(2-ethyl)hexoxyvalerolactone (C2 EtHexVL, 746 mg, 44\% yield) as a colorless, highly viscous oil.

${ }^{1} \mathbf{H}$ NMR $\left(500 \mathrm{MHz}, \mathrm{CDCl}_{3}\right) \delta 4.51$ (ddd, $\left.J=11.5,5.2,1.0 \mathrm{~Hz}, 1 \mathrm{H},(\mathrm{CO}) \mathrm{O}-\mathrm{CH}_{e q} \mathrm{H}_{\mathrm{ax}} \mathrm{CH}\right), 4.48$ $\left(\mathrm{dd}, J=11.5,7.8 \mathrm{~Hz}, 1 \mathrm{H},(\mathrm{CO}) \mathrm{O}-\mathrm{CH}_{\mathrm{eq}} H_{a x} \mathrm{CH}\right), 4.08\left(\mathrm{dd}, J=10.9,5.7 \mathrm{~Hz}, 1 \mathrm{H}, \mathrm{CHCO}_{2} \mathrm{CH}_{a} \mathrm{H}_{\mathrm{b}}\right)$, $4.05\left(\mathrm{dd}, J=11.0,5.9 \mathrm{~Hz}, 1 \mathrm{H}, \mathrm{CHCO}_{2} \mathrm{CH}_{\mathrm{a}} \mathrm{H}_{b}\right), 2.93(\mathrm{dddd}, J=7.8,7.8,6.8,5.2 \mathrm{~Hz}, 1 \mathrm{H}, \mathrm{CH}$ $\mathrm{CO}_{2} \mathrm{Alk}$ ), 2.69 (ddd, $J=17.6,6.9,6.9 \mathrm{~Hz}, 1 \mathrm{H},-\mathrm{CH}_{c} \mathrm{H}_{\mathrm{d}}(\mathrm{CO}) \mathrm{OCH}_{2}$ ), 2.57 (ddd, $J=17.6,7.5,7.5$ $\mathrm{Hz}, 1 \mathrm{H},-\mathrm{CH}_{\mathrm{c}} \mathrm{H}_{d}(\mathrm{CO}) \mathrm{OCH}_{2}$ ), 2.23 (ddd, $1 \mathrm{H}, \mathrm{J}=13.9,7.6,7.6,7.6 \mathrm{~Hz},-\mathrm{O}_{2} \mathrm{CCH}_{2} \mathrm{CH}_{\mathrm{eq}} \mathrm{H}_{a x} \mathrm{CH}-$ ), 2.19 (dddd, $\left.J=13.9,7.6,7.6,6.7,0.9 \mathrm{~Hz}, 1 \mathrm{H},-\mathrm{O}_{2} \mathrm{CCH}_{2} \mathrm{CH}_{e q} \mathrm{H}_{\mathrm{ax}} \mathrm{CH}\right), 1.63-1.56(\mathrm{~m}, 1 \mathrm{H}$, $\left.\mathrm{CHCO}_{2} \mathrm{CH}_{2} \mathrm{CH}\right), 1.39-1.24(\mathrm{~m}, 8 \mathrm{H})$, and $0.90\left[\mathrm{t}, \mathrm{J}=7.3 \mathrm{~Hz}, 6 \mathrm{H},\left(\mathrm{CH}_{2} \mathrm{CH}_{3}\right)_{\mathrm{a}}\right.$ and $\left.\left(\mathrm{CH}_{2} \mathrm{CH}_{3}\right)_{\mathrm{b}}\right]$.

${ }^{13} \mathrm{C}$ NMR $\left(125 \mathrm{MHz}, \mathrm{CDCl}_{3}\right) \delta 171.5,170.5,68.9,68.0,38.8,38.4,30.5,29.0,28.2,23.9,23.1$, 21.8, 14.2, and 11.1.

IR (neat, selected peaks): 2959, 2930, 2873, 2861, 1733, 1461, 1242, and $1196 \mathrm{~cm}^{-1}$.

HRMS: Calculated for $\left(\mathrm{C}_{14} \mathrm{H}_{24} \mathrm{O}_{4} \mathrm{Na}\right)^{+} 279.1567$; found: 279.1565 . 


\section{4-Carboundecoxyvalerolactone (undecyl 6-oxotetrahydro-2H-pyran-3-carboxylate, $\mathrm{CC}_{11} \mathrm{VL}$ )}

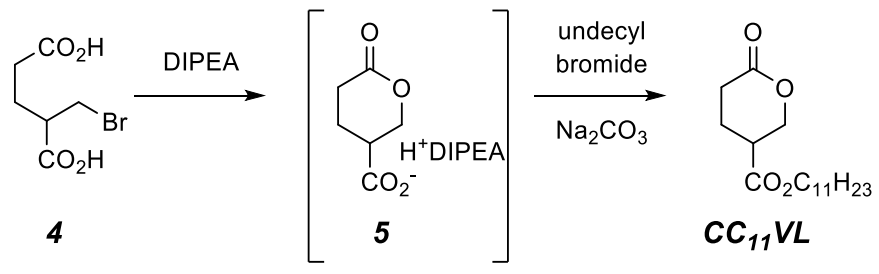

2-(Bromomethyl)glutaric acid (4, 1.0 equiv, $1.50 \mathrm{~g}, 6.67 \mathrm{mmol})$ and a stir bar were placed into a $100-\mathrm{mL}$ culture tube. DCM $(20 \mathrm{~mL})$ was added followed by Hünig's base (3 equiv, $3.5 \mathrm{~mL}, 20$ mmol). The reaction mixture was stirred at ambient temperature for $20 \mathrm{~h}$ and concentrated in vacuo to afford a white slurry. The slurry was resuspended in DMF $(20 \mathrm{~mL})$ and 1bromoundecane (1.5 equiv, $2.4 \mathrm{~mL}, 10 \mathrm{mmol}$ ) followed by $\mathrm{Na}_{2} \mathrm{CO}_{3}$ (1.5 equiv, $\left.1.06 \mathrm{~g}, 10 \mathrm{mmol}\right)$ were added. The heterogeneous mixture was stirred at ambient temperature for $24 \mathrm{~h}$ followed by $35^{\circ} \mathrm{C}$ for $20 \mathrm{~h}$ to achieve full conversion. The mixture was concentrated in vacuo, and the white residue was partitioned into EtOAc (ca. $80 \mathrm{~mL}$ ) and water (ca. $30 \mathrm{~mL}$ ). The organic layer was washed with $\mathrm{NH}_{4} \mathrm{Cl}, \mathrm{NaHCO}_{3}$, and brine; dried with $\mathrm{MgSO}_{4}$; and concentrated in vacuo to a leave a yellow oil. The oil was further purified via column chromatography (silica gel, 1:3 EtOAc:Hex) to afford 4-carboundecoxyvalerolactone $\left(\mathbf{C C}_{\mathbf{1 1}} \mathbf{V L}, 825 \mathrm{mg}, 42 \%\right.$ yield) as a colorless, highly viscous oil.

${ }^{1} \mathbf{H}$ NMR $\left(500 \mathrm{MHz}, \mathrm{CDCl}_{3}\right) \delta 4.50\left(\mathrm{ddd}, J=11.5,5.2,1.0 \mathrm{~Hz}, 1 \mathrm{H},(\mathrm{CO}) \mathrm{O}-\mathrm{CH}_{e q} \mathrm{H}_{\mathrm{ax}} \mathrm{CH}\right), 4.47$ $\left(\mathrm{dd}, J=11.5,7.7 \mathrm{~Hz}, 1 \mathrm{H},(\mathrm{CO}) \mathrm{O}-\mathrm{CH}_{\mathrm{eq}} H_{a x} \mathrm{CH}\right), 4.13\left(\mathrm{t}, J=6.7 \mathrm{~Hz}, 1 \mathrm{H}, \mathrm{CO}_{2} \mathrm{CH}_{2} \mathrm{C}_{10} \mathrm{H}_{21}\right), 2.92$ (dddd, $\left.J=7.8,7.8,6.6,5.2 \mathrm{~Hz}, 1 \mathrm{H}, \mathrm{CH}-\mathrm{CO}_{2} \mathrm{Alk}\right), 2.69(\mathrm{ddd}, J=17.6,6.9,6.9 \mathrm{~Hz}, 1 \mathrm{H}$, $\mathrm{CH}_{a} \mathrm{H}_{\mathrm{b}}(\mathrm{CO}) \mathrm{OCH}_{2}$ ), 2.56 (ddd, $J=17.6,7.5,7.5 \mathrm{~Hz}, 1 \mathrm{H},-\mathrm{CH}_{\mathrm{a}} \mathrm{H}_{b} \mathrm{CO}_{2} \mathrm{CH}_{2}$ ), 2.23 (dddd, $1 \mathrm{H}, J=$ $13.9,7.6,7.6,7.6 \mathrm{~Hz}, \mathrm{O}_{2} \mathrm{CCH}_{2} \mathrm{H}_{\mathrm{eq}} \mathrm{CH}_{a x} \mathrm{CH}-$ ), 2.19 (dddd, $J=14.0,7.6,7.6,6.7,1.0 \mathrm{~Hz}, 1 \mathrm{H}$, $\mathrm{O}_{2} \mathrm{CCH}_{2} \mathrm{H}_{e a} \mathrm{CH}_{\mathrm{ax}} \mathrm{CH}$ ), 1.64 (br app pent, $J=6.8 \mathrm{~Hz}, 2 \mathrm{H}, \mathrm{CO}_{2} \mathrm{CH}_{2} \mathrm{CH}_{2}$ ), 1.38-1.21 (m, 16H), and $0.88\left(\mathrm{t}, J=7.0 \mathrm{~Hz}, 3 \mathrm{H}, \mathrm{CH}_{3}\right)$

${ }^{13}$ C NMR $\left(125 \mathrm{MHz}, \mathrm{CDCl}_{3}\right) \delta 171.4,170.5,68.9,65.8,38.3,32.0,29.71,29.68,29.6,29.4$, 29.3, 28.6, 28.3, 26.0, 22.8, 21.8, and 14.3.

IR (neat, selected peaks): 2954, 2922, 2853, 1731, 1459, 1382, 1333, 1301, 1242, and $1058 \mathrm{~cm}^{-1}$.

HRMS: Calculated for $\left(\mathrm{C}_{17} \mathrm{H}_{30} \mathrm{O}_{4} \mathrm{Na}\right)^{+} 321.2036$; found: 321.2028 . 


\section{Preparation and Characterization of Polymers}

Poly(4-Carbomethoxyvalerolactone) (PCMeVL)

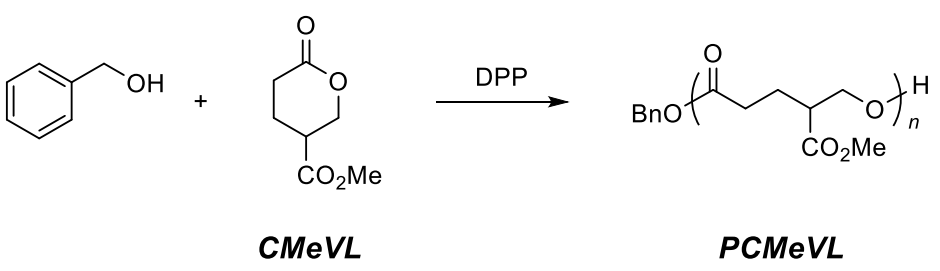

A low molecular weight sample (ca. $8 \mathrm{~kg} \mathrm{~mol}^{-1}$ ) of PCMeVL was prepared by the previously reported procedure. ${ }^{3}$

\section{A higher molecular weight sample was prepared by the following procedure, which is essentially the same procedure used to synthesize higher MW polymers from four of the other monomers.}

Benzyl alcohol (1.0 equiv, $8.9 \mu \mathrm{L}, 0.030 \mathrm{mmol})$, diphenyl phosphate [DPP, $\left(\mathrm{PhO}_{2}\right) \mathrm{PO}_{2} \mathrm{H}, 3.50$ equiv, $75.8 \mathrm{mg}, 0.300 \mathrm{mmol}$ ], and CMeVL (700 equiv, $9.503 \mathrm{~g}$, $60.13 \mathrm{mmol}$, target polymer $M_{\mathrm{n}}$ $=111 \mathrm{~kg} \mathrm{~mol}^{-1}$ ), and a stir bar were sequentially added to a $50-\mathrm{mL}$ culture tube in a nitrogen filled glovebox. The tube was sealed with a Teflon-lined cap and the mixture was stirred for $20 \mathrm{~h}$ at $70{ }^{\circ} \mathrm{C}$. The tube was opened and DCM (ca. $30 \mathrm{~mL}$ ) and $\mathrm{NEt}_{3}(\mathrm{ca} .2 \mathrm{~mL}$ ) were added to the solidified reaction mass. The tube was capped and the suspension was heated at $70{ }^{\circ} \mathrm{C}$ until the polymer was fully dissolved (ca. $16 \mathrm{~h})$. $\left({ }^{1} \mathrm{H}\right.$ NMR analysis of an aliquot of the dissolved solution prior to precipitation showed $92 \%$ monomer conversion). The polymer was precipitated by dropwise addition into cooled, stirred $\mathrm{MeOH}$ (ca. $300 \mathrm{~mL}$ ) in a 1-L Erlenmeyer flask. The contents were allowed to settle and after $15 \mathrm{~min}$ the supernatant was decanted, DCM (ca. $30 \mathrm{~mL}$ ) and $\mathrm{NEt}_{3}$ (ca. $0.1 \mathrm{~mL}$ ) were added to redissolve the polymer, and $\mathrm{MeOH}$ (ca. $300 \mathrm{~mL}$ ) was slowly added to the stirred polymer/DCM solution to reprecipitate the polymer. The sample was once again cooled $\left(0^{\circ} \mathrm{C}\right)$ and allowed to stand $(30 \mathrm{~min})$, and the supernatant was decanted. The white-solid sample was placed under vacuum (ca. $0.1 \mathrm{mmHg}$ ) in a PTFE-lined aluminum pan at $110^{\circ} \mathrm{C}$ for four hours to give poly(4-carbomethoxyvalerolactone) (PCMeVL, $8.36 \mathrm{~g}, 77 \%$ yield) as a white, cloudy disk. This sample was melt pressed $\left(120^{\circ} \mathrm{C}, 5\right.$ minutes $)$ and annealed at $35^{\circ} \mathrm{C}$ ( $24 \mathrm{~h}$ ) and then at RT (3 days) prior to its evaluation in mechanical testing.

${ }^{1} \mathbf{H}$ NMR $\left(500 \mathrm{MHz}, \mathrm{CDCl}_{3}\right): \delta 7.39-7.31(\mathrm{~m}, 5 \mathrm{H}, \mathrm{Ar}), 5.12\left(\mathrm{~s}, 2 \mathrm{H},-\mathrm{OCH}_{2}-\mathrm{Ar}-\mathrm{CH}_{2} \mathrm{O}-\right), 4.23$ [d, $J=6.3 \mathrm{~Hz}, 1222 \mathrm{H}\left(611\right.$ repeat units (RUs), $\mathrm{CO}_{2} \mathrm{CH}_{2}{ }^{\mathrm{RU}}$ ], 3.71 [s, $1830 \mathrm{H}$ (610 RUs), $\mathrm{CO}_{2} \mathrm{CH}_{3}{ }^{\mathrm{RU}}$ ], 2.75 [dddd, $J=8.7,6.0,6.0,6.0 \mathrm{~Hz}, 602 \mathrm{H}(602 \mathrm{RUs})-C H \mathrm{CO}_{2} \mathrm{Me}^{\mathrm{RU}}$ ], 2.39 [ddd, $J=16.7,8.6$, $6.6 \mathrm{~Hz}, 605 \mathrm{H}(605 \mathrm{RU}),-\mathrm{O}_{2} \mathrm{CCH}_{a} \mathrm{H}_{\mathrm{b}} \mathrm{CH}_{2}{ }^{\mathrm{RU}}$ ], 2.35 [ddd, $J=16.7,7.5,7.5 \mathrm{~Hz}, 604 \mathrm{H}(604 \mathrm{RU}),-$ $\mathrm{O}_{2} \mathrm{CCH}_{\mathrm{a}} \mathrm{H}_{\mathrm{b}} \mathrm{CH}_{2}{ }^{\mathrm{RU}}$ ], 1.92 [dddd, $J=14.2,8.2,8.2,6.6 \mathrm{~Hz}, 586 \mathrm{H}$ (586 RUs), $-\mathrm{O}_{2} \mathrm{CCH}_{2} \mathrm{CH}_{\mathrm{a}} \mathrm{H}_{\mathrm{b}}{ }^{\mathrm{RU}}$ ], and 1.87 [dddd, $J=14.0,8.5,7.2,5.4 \mathrm{~Hz}, 616 \mathrm{H}(616 \mathrm{RU}),-\mathrm{O}_{2} \mathrm{CCH}_{2} \mathrm{CH}_{\mathrm{a}} H_{b}{ }^{\mathrm{RU}}$ ]. $M_{\mathrm{n}}(\mathrm{NMR})=97$ $\mathrm{kg} \mathrm{mol}^{-1}$ (from analysis of the benzylic protons in the incorporated initiator). These data are quite consistent with those for a $71 \mathrm{~kg} \mathrm{~mol}^{-1}$ sample previously reported. ${ }^{3}$

The ${ }^{13} \mathbf{C}$ NMR and IR spectra and TGA thermogram were consistent with those previously reported. ${ }^{3}$

SEC (THF, MALS): $M_{\mathrm{n}}=116 \mathrm{~kg} \mathrm{~mol}^{-1}, M_{\mathrm{w}}=130 \mathrm{~kg} \mathrm{~mol}^{-1}, Ð=1.1, d n / d c=0.0543$

$\operatorname{DSC}\left(10{ }^{\circ} \mathrm{C} \mathrm{min}{ }^{-1}\right): T_{\mathrm{g}}=-10{ }^{\circ} \mathrm{C}, T_{\mathrm{m}}=49,78^{\circ} \mathrm{C}\left(\Delta H_{\mathrm{m}}=35.4 \mathrm{~J} \mathrm{~mol}^{-1}\right)$ 


\section{Poly(4-Carboethoxyvalerolactone) (PCEtVL)}

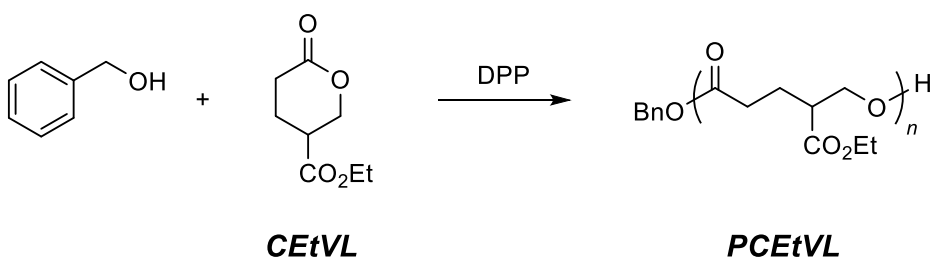

In a nitrogen-filled glovebox, $\mathrm{BnOH}(1.0$ equiv, $3.1 \mu \mathrm{L}, 0.030 \mathrm{mmol})$, diphenyl phosphate [DPP, $(\mathrm{PhO})_{2} \mathrm{PO}_{2} \mathrm{H}, 0.50$ equiv, $\left.3.8 \mathrm{mg}, 0.015 \mathrm{mmol}\right]$, 4-carboethoxyvalerolactone (CEtVL, 50.0 equiv, $258 \mathrm{mg}, 1.50 \mathrm{mmol}$ ), and a stir bar were added sequentially to a 1-dram vial. The vial was sealed with a Teflon-lined cap and the mixture was stirred for $23 \mathrm{~h}$ at ambient temperature. At this time, an aliquot (ca. $3 \mathrm{mg}$ ) was dissolved in $\mathrm{CDCl}_{3}\left(0.55 \mathrm{~mL}\right.$ ) with $\mathrm{NEt}_{3}($ ca. $1 \mu \mathrm{L}$ ) and analyzed via ${ }^{1} \mathrm{H}$ NMR spectroscopy, which indicated nearly complete monomer conversion. The vial was removed from the glovebox and the sample was dissolved in $\mathrm{NEt}_{3}$ (ca. $10 \mu \mathrm{L}$ ) and DCM (1-2 mL). The polymer was precipitated by dropwise addition into stirred $\mathrm{MeOH}(15 \mathrm{~mL})$. The vial was cooled $\left(0^{\circ} \mathrm{C}\right)$ and centrifuged, and the supernatant was decanted. DCM (ca. $\left.1 \mathrm{~mL}\right)$ was added to redissolve the polymer and $\mathrm{MeOH}$ (ca. $15 \mathrm{~mL}$ ) was slowly added to the stirred polymer/DCM solution to reprecipitate the polymer. The sample was once again cooled $\left(0{ }^{\circ} \mathrm{C}\right)$, centrifuged, decanted, and placed under vacuum (ca. $0.1 \mathrm{mmHg}$ ) at $60{ }^{\circ} \mathrm{C}$ to give poly(4carboethoxyvalerolactone) (PCEtVL, $202 \mathrm{mg}, 77 \%$ yield) as a slightly cloudy, waxy solid.

${ }^{1} \mathbf{H}$ NMR (500 MHz, $\mathrm{CDCl}_{3}$ ) See i) the annotated spectrum and ii) the detailed line listing for PCMeVL above for the coupling constants typically seen for this family of polyesters. $M_{\mathrm{n}}=8.2$ $\mathrm{kg} \mathrm{mol}^{-1}$ (from analysis of the benzylic protons in the incorporated initiator).

${ }^{13}$ C NMR $\left(125 \mathrm{MHz}, \mathrm{CDCl}_{3}\right) \delta$ 172.6, 172.2, 64.5, 61.0, 44.0, 31.3, 23.7, and 14.3.

IR (neat, selected peaks): 2980, 2909, 1725 (strong), 1453, 1378, 1151 (strong), and $1020 \mathrm{~cm}^{-1}$.

SEC (THF, MALS): $M_{\mathrm{n}}=14.5 \mathrm{~kg} \mathrm{~mol}^{-1}, M_{\mathrm{w}}=15.5 \mathrm{~kg} \mathrm{~mol}^{-1}, Ð=1.06, d n / d c=0.0598$.

TGA $T_{\mathrm{d}} 1 \%=218^{\circ} \mathrm{C}, 5 \%=249^{\circ} \mathrm{C}$.

DSC Cooling and ramping rates of $5^{\circ} \mathrm{C} / \mathrm{min}: T_{\mathrm{g}}=-29^{\circ} \mathrm{C}\left[T_{\mathrm{m}}=52{ }^{\circ} \mathrm{C}\right.$ on initial heating cycle, $\left.\left(\Delta H_{\mathrm{m}}=6.4 \mathrm{~J} \mathrm{~mol}^{-1}\right)\right]$

Cooling and ramping rates of $10{ }^{\circ} \mathrm{C} / \mathrm{min}: T_{\mathrm{g}}=-28^{\circ} \mathrm{C}$

A higher molar mass sample was prepared in a similar fashion to PCMeVL using BnOH (1 equiv, $6.6 \mu \mathrm{L}, 0.064 \mathrm{mmol}$ ), CEtVL (640 equiv, $7.00 \mathrm{~g}$, $40.7 \mathrm{mmol}$ ), and DPP (3 equiv, 48.1 $\mathrm{mg}, 0.191 \mathrm{mmol})$. The polymer was heated to $70{ }^{\circ} \mathrm{C}$ for $22 \mathrm{~h}(93 \%$ monomer conversion), precipitated, and heated under vacuum as described for PCMeVL to give PCEtVL was a colorless, highly viscous liquid that solidified to a white, cloudy disk over three days at room temperature $(5.45 \mathrm{~g}, 78 \%$ yield).

${ }^{1} \mathrm{H}$ NMR of higher MW sample $M_{\mathrm{n}}=110 \mathrm{~kg} \mathrm{~mol}^{-1}$ (from analysis of the benzylic protons in the incorporated initiator) or $82 \mathrm{~kg} \mathrm{~mol}^{-1}$ (integrated against the $\mathrm{CH}_{2} \mathrm{OH}$ end group).

SEC of higher MW sample (THF, MALS): $M_{\mathrm{n}}=76.1 \mathrm{~kg} \mathrm{~mol}^{-1}, M_{\mathrm{w}}=86.3 \mathrm{~kg} \mathrm{~mol}^{-1}, Ð=1.14$, $d n / d c=0.0643$.

$\operatorname{DSC}\left(10{ }^{\circ} \mathrm{C} \mathrm{min}{ }^{-1}\right): T_{\mathrm{g}}=-26{ }^{\circ} \mathrm{C}, T_{\mathrm{m}}=50{ }^{\circ} \mathrm{C}\left(\Delta H_{\mathrm{m}}=13.0 \mathrm{~J} \mathrm{~mol}^{-1}\right)$. 


\section{Poly(4-Carbo-iso-propoxyvalerolactone) (PCiPrVL)}

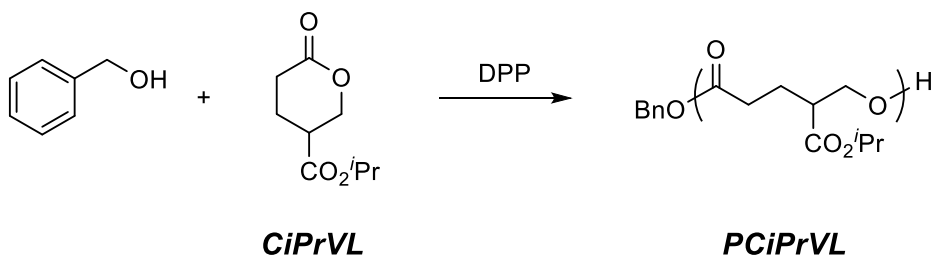

In a nitrogen-filled glovebox, $\mathrm{BnOH}(1.0$ equiv, $3.1 \mu \mathrm{L}, 0.030 \mathrm{mmol})$, diphenyl phosphate [DPP, $(\mathrm{PhO})_{2} \mathrm{PO}_{2} \mathrm{H}, 0.50$ equiv, $3.8 \mathrm{mg}, 0.015 \mathrm{mmol}$ ], 4-carboisopropoxyvalerolactone (CiPrVL, 50.0 equiv, $279 \mathrm{mg}, 1.50 \mathrm{mmol}$ ), and a stir bar were added sequentially to a 1-dram vial. The vial was sealed with a Teflon-lined cap and the mixture was stirred for $24 \mathrm{~h}$ at ambient temperature. At this time, an aliquot of this waxy substance (ca. $3 \mathrm{mg}$ ) was dissolved in $\mathrm{CDCl}_{3}(0.55 \mathrm{~mL})$ with $\mathrm{NEt}_{3}($ ca. $1 \mu \mathrm{L})$ and analyzed via ${ }^{1} \mathrm{H}$ NMR spectroscopy. The monomer conversion was observed to be $98 \%$. The vial was removed from the glovebox and the sample was dissolved in $\mathrm{NEt}_{3}$ (ca. $10 \mu \mathrm{L})$ and DCM (1-2 mL). The polymer was precipitated by dropwise addition of this solution into stirred $\mathrm{MeOH}(15 \mathrm{~mL})$. The vial was cooled $\left(0^{\circ} \mathrm{C}\right)$ and centrifuged, and the supernatant was decanted. DCM (ca. $1 \mathrm{~mL}$ ) was added to redissolve the polymer and $\mathrm{MeOH}$ (ca. $15 \mathrm{~mL}$ ) was slowly added to the stirred polymer/DCM solution to reprecipitate the polymer. The sample was once again cooled $\left(0^{\circ} \mathrm{C}\right)$, the suspension centrifuged, the supernatant liquid decanted, and the remaining solid placed under vacuum (ca. $0.1 \mathrm{mmHg}$ ) at $60{ }^{\circ} \mathrm{C}$ to give poly(4carboisopropoxyvalerolactone) (PCiPrVL, $243 \mathrm{mg}$, 86\% yield) as a cloudy, waxy solid.

${ }^{1} \mathbf{H}$ NMR (500 MHz, $\mathrm{CDCl}_{3}$ ) See i) the annotated spectrum and ii) the detailed line listing for PCMeVL above for the coupling constants typically seen for this family of polyesters. $M_{\mathrm{n}}=8.9$ $\mathrm{kg} \mathrm{mol}^{-1}$ (from analysis of the benzylic protons in the incorporated initiator).

${ }^{13} \mathrm{C}$ NMR $\left(125 \mathrm{MHz}, \mathrm{CDCl}_{3}\right) \delta 172.3,172.2,68.5,64.6,44.2,31.4,23.8$, and $21.9(2 \mathrm{x})$.

IR (neat, selected peaks): 2981, 2942, 1724 (strong), 1455, 1385, 1244, 1156 (strong), and 1052 (strong) $\mathrm{cm}^{-1}$.

SEC (THF, MALS): $M_{\mathrm{n}}=14.9 \mathrm{~kg} \mathrm{~mol}^{-1}, M_{\mathrm{w}}=15.4 \mathrm{~kg} \mathrm{~mol}^{-1}, Ð=1.0(3), d n / d c=0.0571$.

TGA $T_{\mathrm{d}} 1 \%=228^{\circ} \mathrm{C}, 5 \%=269^{\circ} \mathrm{C}$.

DSC Cooling and ramping rates of $5{ }^{\circ} \mathrm{C} / \mathrm{min}: T_{\mathrm{g}}=-24^{\circ} \mathrm{C}, T_{\mathrm{c}}=36^{\circ} \mathrm{C}, T_{\mathrm{m}}=62{ }^{\circ} \mathrm{C},\left(\Delta H_{\mathrm{m}}=\right.$ $\left.19.8 \mathrm{~J} \mathrm{~mol}^{-1}\right)$.

Cooling and ramping rates of $10^{\circ} \mathrm{C} / \mathrm{min}: T_{\mathrm{g}}=-22{ }^{\circ} \mathrm{C}, T_{\mathrm{c}}=49{ }^{\circ} \mathrm{C}, T_{\mathrm{m}}=67^{\circ} \mathrm{C}\left(\Delta H_{\mathrm{m}}=3.6\right.$ $\mathrm{J} \mathrm{mol}^{-1}$ )

At $10{ }^{\circ} \mathrm{C} \min ^{-1}$ the endotherm related to the melting temperature was substantially reduced compared to the $5^{\circ} \mathrm{C} / \mathrm{min}$ heating rate. 
A higher molar mass sample was prepared in a similar fashion to that for PCMeVL using BnOH (1 equiv, $4.7 \mu \mathrm{L}, 0.046 \mathrm{mmol}$ ), CiPrVL (591 equiv, $5.00 \mathrm{~g}, 26.9 \mathrm{mmol}$ ), and DPP (3 equiv, 34.4 $\mathrm{mg}, 0.14 \mathrm{mmol})$. The polymer was heated to $70{ }^{\circ} \mathrm{C}$ for $7 \mathrm{~h}(90 \%$ monomer conversion $)$, precipitated, and heated under vacuum as described for PCMeVL to give PCiPrVL as a white, cloudy disk (3.21 g, 64\% yield).

${ }^{1} \mathbf{H}$ NMR $\left(500 \mathrm{MHz}, \mathrm{CDCl}_{3}\right) M_{\mathrm{n}}=78 \mathrm{~kg} \mathrm{~mol}^{-1}$ (from analysis of the benzylic protons in the incorporated initiator).

SEC of higher MW (THF, MALS): $M_{\mathrm{n}}=56.6 \mathrm{~kg} \mathrm{~mol}^{-1}, M_{\mathrm{w}}=64.8 \mathrm{~kg} \mathrm{~mol}^{-1}, Ð=1.15, d n / d c=$ 0.0639 .

$\operatorname{DSC}\left(10{ }^{\circ} \mathrm{C} \mathrm{min}{ }^{-1}\right): T_{\mathrm{g}}=-42{ }^{\circ} \mathrm{C}, T_{\mathrm{m}}=46,56{ }^{\circ} \mathrm{C}\left(\Delta H_{\mathrm{m}}=20.0 \mathrm{~J} \mathrm{~mol}^{-1}\right)$ 


\section{Poly(4-Carbo-n-butoxyvalerolactone) (PCnBuVL)}

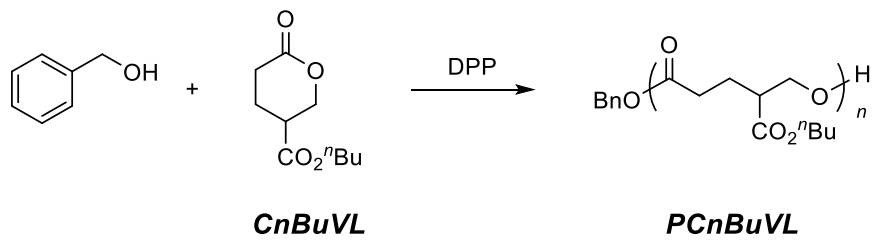

In a nitrogen-filled glovebox, $\mathrm{BnOH}(1.0$ equiv, $3.1 \mu \mathrm{L}, 0.030 \mathrm{mmol})$, diphenyl phosphate [DPP, $(\mathrm{PhO})_{2} \mathrm{PO}_{2} \mathrm{H}, 0.50$ equiv, $4.0 \mathrm{mg}, 0.016 \mathrm{mmol}$ ], 4-carbo- $n$-butoxyvalerolactone $(\mathbf{C n B u V L}, 50.0$ equiv, $300 \mathrm{mg}, 1.50 \mathrm{mmol}$ ), and a stir bar were added sequentially to a 1-dram vial. The vial was sealed with a Teflon-lined cap and the mixture was stirred for $20 \mathrm{~h}$ at ambient temperature. At this time, an aliquot of this waxy sample (ca. $3 \mathrm{mg}$ ) was dissolved in $\mathrm{CDCl}_{3}(0.55 \mathrm{~mL})$ with $\mathrm{NEt}_{3}$ (ca. $1 \mu \mathrm{L}$ ) and analyzed via ${ }^{1} \mathrm{H}$ NMR spectroscopy. The monomer conversion was measured to be $98 \%$. The vial was removed from the glovebox and the sample was dissolved in $\mathrm{NEt}_{3}$ (ca. 10 $\mu \mathrm{L})$ and DCM (1-2 $\mathrm{mL})$. The polymer was precipitated by dropwise addition into stirred $\mathrm{MeOH}$ $(15 \mathrm{~mL})$. The vial was cooled $\left(0^{\circ} \mathrm{C}\right)$ and centrifuged, and the supernatant was decanted. DCM (ca. $1 \mathrm{~mL}$ ) was added to redissolve the polymer and $\mathrm{MeOH}$ (ca. $15 \mathrm{~mL}$ ) was slowly added to the stirred polymer/DCM solution to reprecipitate the polymer. The sample was again cooled $\left(0{ }^{\circ} \mathrm{C}\right)$, centrifuged, decanted, and placed under vacuum (ca. $0.1 \mathrm{mmHg}$ ) at $60{ }^{\circ} \mathrm{C}$ to give poly(4-carbo$n$-butoxyvalerolactone) (PCnBuVL, $235 \mathrm{mg}, 78 \%$ yield) as a cloudy, waxy solid.

${ }^{1} \mathbf{H}$ NMR (500 MHz, $\mathrm{CDCl}_{3}$ ) See i) the annotated spectrum and ii) the detailed line listing for PCMeVL above for the coupling constants typically seen for this family of polyesters. $M_{\mathrm{n}}=9.7$ $\mathrm{kg} \mathrm{mol}^{-1}$ (from analysis of the benzylic protons in the incorporated initiator).

${ }^{13} \mathbf{C}$ NMR $\left(125 \mathrm{MHz}, \mathrm{CDCl}_{3}\right) \delta 172.7,172.3,64.9,64.5,44.1,31.4,30.7,23.7,19.2$, and 13.8. (for the $90 \mathrm{~kg} \mathrm{~mol}^{-1}$ sample).

IR (neat, selected peaks): 2964, 2936, 2876, 1725 (strong), 1447, 1393, 1246, and 1145 (strong) $\mathrm{cm}^{-1}$.

SEC (THF, MALS): $M_{\mathrm{n}}=19.4 \mathrm{~kg} \mathrm{~mol}^{-1}, M_{\mathrm{w}}=28.3 \mathrm{~kg} \mathrm{~mol}^{-1}, Ð=1.4(6), d n / d c=0.0593$.

TGA $T_{\mathrm{d}} 1 \%=252{ }^{\circ} \mathrm{C}, 5 \%=292{ }^{\circ} \mathrm{C}$.

DSC Cooling and heating rate of $5{ }^{\circ} \mathrm{C} / \mathrm{min}: T_{\mathrm{g}}=-44^{\circ} \mathrm{C}, T_{\mathrm{c}}=11^{\circ} \mathrm{C}, T_{\mathrm{m}}=50{ }^{\circ} \mathrm{C}\left(\Delta H_{\mathrm{m}}=30.4\right.$ $\mathrm{J} \mathrm{mol}^{-1}$ ).

Cooling and heating rate of $10{ }^{\circ} \mathrm{C} / \mathrm{min}: T_{\mathrm{g}}=-43{ }^{\circ} \mathrm{C}, T_{\mathrm{c}}=16{ }^{\circ} \mathrm{C} T_{\mathrm{m}}=50{ }^{\circ} \mathrm{C}\left(\Delta H_{\mathrm{m}}=26.1\right.$ $\mathrm{J} \mathrm{mol}^{-1}$ ).

A higher molar mass sample was prepared in a similar fashion to that for PCMeVL using BnOH (1 equiv, $9.5 \mu \mathrm{L}, 0.046 \mathrm{mmol}$ ), CnBuVL (550 equiv, $10.0 \mathrm{~g}, 50.0 \mathrm{mmol}$ ), and DPP (2.8 equiv, $63 \mathrm{mg}, 0.25 \mathrm{mmol})$. The polymer was heated to $70{ }^{\circ} \mathrm{C}$ for $40 \mathrm{~h}(89 \%$ monomer conversion), precipitated, and heated under vacuum as described for PCMeVL to give PCnBuVL as a white, cloudy disk ( $7.24 \mathrm{~g}, 72 \%$ yield $)$.

${ }^{1} \mathbf{H}$ NMR $\left(500 \mathrm{MHz}, \mathrm{CDCl}_{3}\right) M_{\mathrm{n}}=93 \mathrm{~kg} \mathrm{~mol}^{-1}$ (integrated against BnO-) or $900 \mathrm{~kg} \mathrm{~mol}^{-1}$ (integrated against the $\mathrm{CH}_{2} \mathrm{OH}$ end group).

SEC of higher MW (THF, MALS): $M_{\mathrm{n}}=75.0 \mathrm{~kg} \mathrm{~mol}^{-1}, M_{\mathrm{w}}=90.3 \mathrm{~kg} \mathrm{~mol}^{-1}, Ð=1.21, d n / d c=$ 0.0635 .

DSC Heating rate of $10{ }^{\circ} \mathrm{C} / \mathrm{min}: T_{\mathrm{g}}=-42{ }^{\circ} \mathrm{C}, T_{\mathrm{m}}=45^{\circ} \mathrm{C},\left(\Delta H_{\mathrm{m}}=23.5 \mathrm{~J} \mathrm{~mol}^{-1}\right)$. 


\section{Poly(4-Carbo-t-butoxyvalerolactone) (PCtBuVL)}

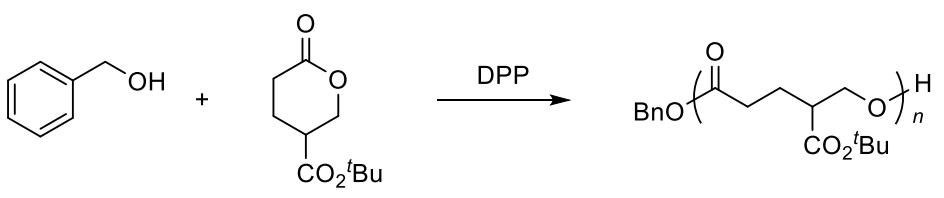

CtBuVL

PCtBuVL

In a nitrogen-filled glovebox, $\mathrm{BnOH}(1.0$ equiv, $3.1 \mu \mathrm{L}, 0.030 \mathrm{mmol})$, diphenyl phosphate [DPP, $(\mathrm{PhO})_{2} \mathrm{PO}_{2} \mathrm{H}, 0.50$ equiv, $\left.4.0 \mathrm{mg}, 0.016 \mathrm{mmol}\right]$, 4-carbo-t-butoxyvalerolactone $(\mathbf{C t B u V L}, 50.0$ equiv, $300 \mathrm{mg}, 1.50 \mathrm{mmol}$ ), and a stir bar were added sequentially to a 1-dram vial. The vial was sealed with a Teflon-lined cap and the mixture was stirred for $20 \mathrm{~h}$ at ambient temperature. At this time, an aliquot (ca. $3 \mathrm{mg}$ ) was dissolved in $\mathrm{CDCl}_{3}(0.55 \mathrm{~mL})$ containing $\mathrm{NEt}_{3}$ (ca. $1 \mu \mathrm{L}$ ) and analyzed via ${ }^{1} \mathrm{H}$ NMR spectroscopy. The monomer conversion was measured to be $97 \%$. The vial was removed from the glovebox and the sample was dissolved in $\mathrm{NEt}_{3}$ (ca. $10 \mu \mathrm{L}$ ) and DCM (1-2 mL). The polymer was precipitated by dropwise addition into stirred $\mathrm{MeOH}(15 \mathrm{~mL})$. The vial was cooled $\left(0{ }^{\circ} \mathrm{C}\right)$ and centrifuged, and the supernatant was decanted. DCM (ca. $\left.1 \mathrm{~mL}\right)$ was added to resuspend the polymer and $\mathrm{MeOH}$ (ca. $15 \mathrm{~mL}$ ) was slowly added to the stirred polymer/DCM solution to reprecipitate the polymer. This precipitation was repeated two more times because the chilled polymer/precipitation residue appeared to hold significant amounts of solvent. The four precipitations afforded poly(4-carbo-t-butoxyvalerolactone) (PCtBuVL, 98 $\mathrm{mg}, 32 \%$ yield) as a cloudy, hard solid.

${ }^{1} \mathbf{H}$ NMR (500 MHz, $\mathrm{CDCl}_{3}$ ) See i) the annotated spectrum and ii) the detailed line listing for PCMeVL above for the coupling constants typically seen for this family of polyesters. $M_{\mathrm{n}}=11.7$ $\mathrm{kg} \mathrm{mol}^{-1}$ (from analysis of the benzylic protons in the incorporated initiator).

${ }^{13}$ C NMR (125 MHz, $\left.\mathrm{CDCl}_{3}\right) \delta$ 172.3, 171.9, 81.4, 64.8, 45.0, 31.4, 28.2, and 24.9.

IR (neat, selected peaks): 2978, 2935, 1722 (strong), 1457, 1392, 1366, 1247, 1143 (strong), 1055 , and $846 \mathrm{~cm}^{-1}$.

SEC (THF, MALS): $M_{\mathrm{n}}=15.6 \mathrm{~kg} \mathrm{~mol}^{-1}, M_{\mathrm{w}}=18.0 \mathrm{~kg} \mathrm{~mol}^{-1}, Ð=1.15, d n / d c=0.0431$.

TGA $T_{\mathrm{d}} 1 \%=197{ }^{\circ} \mathrm{C}, 5 \%=214^{\circ} \mathrm{C}$.

DSC Cooling and heating rate of $5^{\circ} \mathrm{C} / \mathrm{min}: T_{\mathrm{g}}=-7{ }^{\circ} \mathrm{C}, T_{\mathrm{c}}=44$ (on cool) ${ }^{\circ} \mathrm{C}, T_{\mathrm{m}}=95^{\circ} \mathrm{C}\left(\Delta H_{\mathrm{m}}=\right.$ $30.7 \mathrm{~J} \mathrm{~mol}^{-1}$ ).

Cooling and heating rate of $10{ }^{\circ} \mathrm{C} / \mathrm{min}$ : $T_{\mathrm{g}}=-6^{\circ} \mathrm{C}, T_{\mathrm{c}}=36{ }^{\circ} \mathrm{C}$ (on cool), 37 (on ramp) ${ }^{\circ} \mathrm{C}$, $T_{\mathrm{m}}=95^{\circ} \mathrm{C}\left(\Delta H_{\mathrm{m}}=31.0 \mathrm{~J} \mathrm{~mol}^{-1}\right)$. 


\section{Poly(4-Carbobenzoxyvalerolactone) (PCBnVL)}

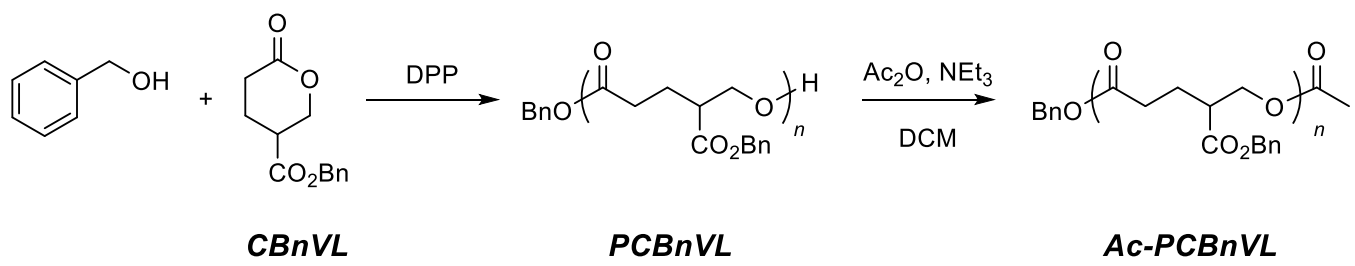

In a nitrogen-filled glovebox, $\mathrm{BnOH}(1.0$ equiv, $5.3 \mu \mathrm{L}, 0.051 \mathrm{mmol})$, diphenyl phosphate [DPP, $(\mathrm{PhO})_{2} \mathrm{PO}_{2} \mathrm{H}, 0.50$ equiv, $\left.6.0 \mathrm{mg}, 0.024 \mathrm{mmol}\right]$, 4-carbobenzoxyvalerolactone $(\mathbf{C B n V L}, 50.0$ equiv, $600 \mathrm{mg}, 2.56 \mathrm{mmol}$ ), and a stir bar were added sequentially to a 1-dram vial. The vial was sealed with a Teflon-lined cap and the mixture was stirred for $24 \mathrm{~h}$ at ambient temperature. An aliquot of this viscous liquid sample (ca. $3 \mathrm{mg}$ ) was dissolved in $\mathrm{CDCl}_{3}(0.55 \mathrm{~mL})$ containing $\mathrm{NEt}_{3}$ (ca. $1 \mu \mathrm{L}$ ) and analyzed via ${ }^{1} \mathrm{H}$ NMR spectroscopy. The monomer conversion was measured to be $95 \%$. The vial was removed from the glovebox and the sample was dissolved in $\mathrm{DCM}(2 \mathrm{~mL}) . \mathrm{NEt}_{3}(100 \mu \mathrm{L})$ and $\mathrm{Ac}_{2} \mathrm{O}(100 \mu \mathrm{L})$ were added and the solution was stirred. After $19 \mathrm{~h}$ the polymer was precipitated by dropwise addition into stirred $\mathrm{MeOH}(15 \mathrm{~mL})$. The vial was cooled $\left(0^{\circ} \mathrm{C}\right)$ and centrifuged, and the supernatant was decanted. DCM (ca. $\left.1 \mathrm{~mL}\right)$ was added to dissolve the polymer and $\mathrm{MeOH}$ (ca. $15 \mathrm{~mL}$ ) was slowly added to the stirred solution to reprecipitate the polymer. The sample was once again cooled $\left(0^{\circ} \mathrm{C}\right)$, centrifuged, decanted, and placed under vacuum (ca. $0.1 \mathrm{mmHg}$ ) at $60^{\circ} \mathrm{C}$ to give the end-capped poly $(4-$ carbobenzoxyvalerolactone) (Ac-PCBnVL, $522 \mathrm{mg}, 86 \%$ yield) as a tan, highly viscous liquid that slowly developed some cloudiness after ca. 3 weeks of storage at room temperature.

${ }^{1} \mathbf{H}$ NMR (500 MHz, $\mathrm{CDCl}_{3}$ ) See i) the annotated spectrum and ii) the detailed line listing for PCMeVL above for the coupling constants typically seen for this family of polyesters. $M_{\mathrm{n}}=12.6$ $\mathrm{kg} \mathrm{mol}^{-1}$ (from analysis of the benzylic protons in the incorporated initiator).

${ }^{13}$ C NMR $\left(125 \mathrm{MHz}, \mathrm{CDCl}_{3}\right) \delta 172.5,172.1,135.8,128.7,128.5,128.4,66.8,64.4,44.0,31.2$, and 23.6.

IR (neat, selected peaks): 3089, 3065, 3034, 2955, 1728 (strong), 1454, and 1148 (strong) $\mathrm{cm}^{-1}$.

SEC (THF, MALS): $M_{\mathrm{n}}=10.3 \mathrm{~kg} \mathrm{~mol}^{-1}, M_{\mathrm{w}}=10.9 \mathrm{~kg} \mathrm{~mol}^{-1}, Ð=1.05, d n / d c=0.1190$.

TGA $T_{\mathrm{d}} 1 \%=289^{\circ} \mathrm{C}, 5 \%=307{ }^{\circ} \mathrm{C}$.

DSC Cooling and heating rate of $5{ }^{\circ} \mathrm{C} / \mathrm{min}: T_{\mathrm{g}}=-6^{\circ} \mathrm{C}\left[T_{\mathrm{m}}=47^{\circ} \mathrm{C}\right.$ on initial heating cycle, $\left.4.4 \mathrm{~J} \mathrm{~mol}^{-1}\right]^{*}$

Cooling and heating rate of $10{ }^{\circ} \mathrm{C} / \mathrm{min}: T_{\mathrm{g}}=-6^{\circ} \mathrm{C}$.

*An SEC, taken two days after the preparation of PCBnVL, showed no presence of a melting characteristic. However, an SEC one month after its synthesis displayed a clear melting phenomenon at $47^{\circ} \mathrm{C}$. 


\section{Poly[4-Carbo(2-ethylhexoxy)valerolactone] (PC ${ }^{2}$ EtHexVL)}

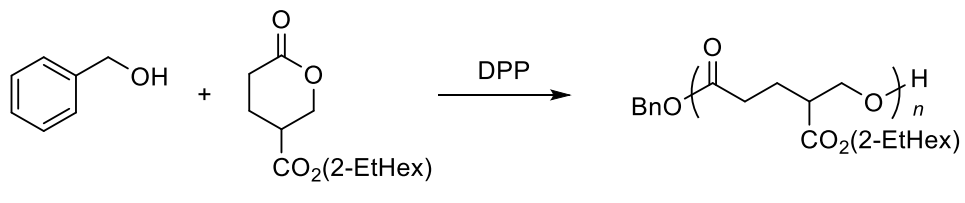

$C^{2} E t H e x V L$

$P C^{2}$ EtHexVL

Benzyl alcohol (1.0 equiv, $2.1 \mu \mathrm{L}, 0.020 \mathrm{mmol})$, diphenyl phosphate [DPP, $(\mathrm{PhO})_{2} \mathrm{PO}_{2} \mathrm{H}, 0.50$ equiv, $2.5 \mathrm{mg}, 0.010 \mathrm{mmol}$ ], and $\mathbf{C}^{2} \mathbf{E t H e x V L}$ (50.0 equiv, $256 \mathrm{mg}, 1.00 \mathrm{mmol}$ ), and a stir bar were sequentially added to a 1-dram vial in a nitrogen filled glovebox. The vial was sealed with a Teflon-lined cap and the polymerization was allowed to stir for $19 \mathrm{~h}$. An aliquot of this viscous liquid sample, taken prior to precipitation, showed $97 \%$ monomer conversion via ${ }^{1} \mathrm{H}$ NMR spectroscopy. The procedure for precipitation and heating were followed as described above to give PC ${ }^{2}$ EtHexVL (125 mg, 48\% yield) as a highly viscous liquid.

${ }^{1} \mathbf{H}$ NMR (500 MHz, $\mathrm{CDCl}_{3}$ ) See i) the annotated spectrum and ii) the detailed line listing for PCMeVL above for the coupling constants typically seen for this family of polyesters. $M_{\mathrm{n}}=13.3$ $\mathrm{kg} \mathrm{mol}^{-1}$ (from analysis of the benzylic protons in the incorporated initiator).

${ }^{13} \mathbf{C}$ NMR $\left(125 \mathrm{MHz}, \mathrm{CDCl}_{3}\right) \delta 172.7,172.2,67.4,64.5,44.2,38.8,31.3,30.4(4), 30.4(2), 29.0$, $23.8,23.7,23.0 .14 .2,11.0$. One aliphatic carbon appears as a pair of resonances with very similar chemical shifts, reflecting the mixture of diastereomers.

IR (neat, selected peaks): 2958, 2929, 2873, 2860, 1732 (strong), 1459, 1232, and $1153 \mathrm{~cm}^{-1}$.

SEC (THF, MALS): $M_{\mathrm{n}}=16.0 \mathrm{~kg} \mathrm{~mol}^{-1}, M_{\mathrm{w}}=18.6 \mathrm{~kg} \mathrm{~mol}^{-1}, D=1.21, d n / d c=0.0550$.

TGA $T_{\mathrm{d}} 1 \%=226{ }^{\circ} \mathrm{C}, 5 \%=268^{\circ} \mathrm{C}$.

DSC Cooling and heating rate of $5{ }^{\circ} \mathrm{C} / \mathrm{min}: T_{\mathrm{g}}=-55^{\circ} \mathrm{C}, T_{\mathrm{c}}=-29$ (on cooling) ${ }^{\circ} \mathrm{C}, T_{\mathrm{m}}=-7{ }^{\circ} \mathrm{C}$ $\left(\Delta H_{\mathrm{m}}=13.4 \mathrm{~J} \mathrm{~mol}^{-1}\right)$.

Cooling and heating rate of $10^{\circ} \mathrm{C} / \mathrm{min}: T_{\mathrm{g}}=-54{ }^{\circ} \mathrm{C}, T_{\mathrm{c}}=-31^{\circ} \mathrm{C}$ (on cooling), $37^{\circ} \mathrm{C}$ (on heating $), T_{\mathrm{m}}=-5^{\circ} \mathrm{C}\left(\Delta H_{\mathrm{m}}=9.6 \mathrm{~J} \mathrm{~mol}^{-1}\right)$. 


\section{Poly(4-Carboundecoxyvalerolactone) $\left(\mathrm{PCC}_{11} \mathrm{VL}\right)$}

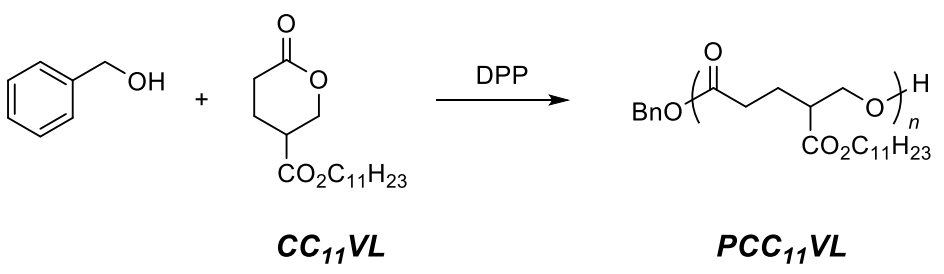

In a nitrogen-filled glovebox, $\mathrm{BnOH}(0.02$ equiv, $2.1 \mu \mathrm{L}, 0.020 \mathrm{mmol})$, diphenyl phosphate [DPP, $(\mathrm{PhO})_{2} \mathrm{PO}_{2} \mathrm{H}, 0.020$ equiv, $5.0 \mathrm{mg}, 0.020 \mathrm{mmol}$ ], 4-carboundecoxyvalerolactone $\left(\mathbf{C C}_{11} \mathbf{V L}, 1\right.$ equiv, $\left.298 \mathrm{mg}, 1.00 \mathrm{mmol}\right)$, and a stir bar were added sequentially to a $1 \mathrm{dram}$. The vial was sealed with a Teflon-lined cap and the mixture was stirred for $23 \mathrm{~h}$ at ambient temperature. An aliquot of this waxy solid sample (ca. $3 \mathrm{mg}$ ) was dissolved in $\mathrm{CDCl}_{3}(0.55 \mathrm{~mL})$ containing $\mathrm{NEt}_{3}$ (ca. $1 \mu \mathrm{L}$ ) and analyzed by ${ }^{1} \mathrm{H}$ NMR spectroscopy. The monomer conversion was measure to be $98 \%$. The vial was removed from the glovebox and the sample was dissolved in $\mathrm{NEt}_{3}$ (ca. $\left.10 \mu \mathrm{L}\right)$ and DCM $(1-2 \mathrm{~mL})$. The polymer was precipitated by dropwise addition into stirred $\mathrm{MeOH}(15 \mathrm{~mL})$. The vial was cooled $\left(0^{\circ} \mathrm{C}\right)$ and centrifuged, and the supernatant was decanted. DCM (ca. $1 \mathrm{~mL}$ ) was added to dissolve the polymer and $\mathrm{MeOH}$ (ca. $15 \mathrm{~mL}$ ) was slowly added to this stirred solution to reprecipitate the polymer. The sample was again cooled $(0$ ${ }^{\circ} \mathrm{C}$ ), centrifuged, decanted, and placed under vacuum (ca. $0.1 \mathrm{mmHg}$ ) at $60{ }^{\circ} \mathrm{C}$ to give poly (4carboundecoxyvalerolactone) (PCC $\mathbf{1 1}_{11}$ VL) as a white, waxy solid (261 $\mathrm{mg}, 87 \%$ yield).

${ }^{1} \mathbf{H}$ NMR (500 MHz, $\mathrm{CDCl}_{3}$ ) See i) the annotated spectrum and ii) the detailed line listing for PCMeVL above for the coupling constants typically seen for this family of polyesters. $M_{\mathrm{n}}=14.1$ $\mathrm{kg} \mathrm{mol}^{-1}$ (from analysis of the benzylic protons in the incorporated initiator).

${ }^{13}$ C NMR $\left(125 \mathrm{MHz}, \mathrm{CDCl}_{3}\right) \delta 172.7,172.3,65.3,64.6,44.1,32.1,31.4,29.8,29.7,29.5$ (2x), 29.4, 28.7, 26.0, 23.7, 22.8, and 14.3.

IR (neat, selected peaks): 2954, 2921, 2853, 1729 (strong), 1465, 1889, and $1575 \mathrm{~cm}^{-1}$.

SEC (THF, MALS): $M_{\mathrm{n}}=13.2 \mathrm{~kg} \mathrm{~mol}^{-1}, M_{\mathrm{w}}=16.4 \mathrm{~kg} \mathrm{~mol}^{-1}, Ð=1.18, d n / d c=0.0614$.

TGA $T_{\mathrm{d}} 1 \%=236{ }^{\circ} \mathrm{C}, 5 \%=268^{\circ} \mathrm{C}$.

DSC Cooling and heating rates of $5^{\circ} \mathrm{C} / \mathrm{min}: T_{\mathrm{g}}=<-75^{\circ} \mathrm{C}, T_{\mathrm{c}}=12{ }^{\circ} \mathrm{C}$ (on cooling), $T_{\mathrm{m}}=55^{\circ} \mathrm{C}$ $\left(\Delta H_{\mathrm{m}}=26.8 \mathrm{~J} \mathrm{~mol}^{-1}\right)$.

Cooling and heating rates of $10^{\circ} \mathrm{C} / \mathrm{min}: T_{\mathrm{g}}=<-75^{\circ} \mathrm{C}, T_{\mathrm{c}}=16^{\circ} \mathrm{C}$ (on cooling), $T_{\mathrm{m}}=55^{\circ} \mathrm{C}$ $\left(\Delta H_{\mathrm{m}}=28.2 \mathrm{~J} \mathrm{~mol}^{-1}\right)$. 


\section{Polymer Testing}

IVa. Matrix-assisted laser desorption ionization time of flight (MALDI-TOF)

A representative analysis is described here. A low molecular sample of PCnBuVL for MALDITOF analysis was prepared using BnOH, CnBuVL, and DPP at $70{ }^{\circ} \mathrm{C}$ for $16 \mathrm{~h}$ (targeted $M_{\mathrm{n}}=\mathrm{ca}$. $\left.2.5 \mathrm{~kg} \mathrm{~mol}^{-1}\right)$. This polymerization reached equilibrium conversion after ca. 2 to $3 \mathrm{~h}$ and was held at $70{ }^{\circ} \mathrm{C}$ for the additional 13 to $14 \mathrm{~h}$ to promote further chain transfer reactions. An expansion of a portion of these data are shown as Figure 6 in the manuscript.

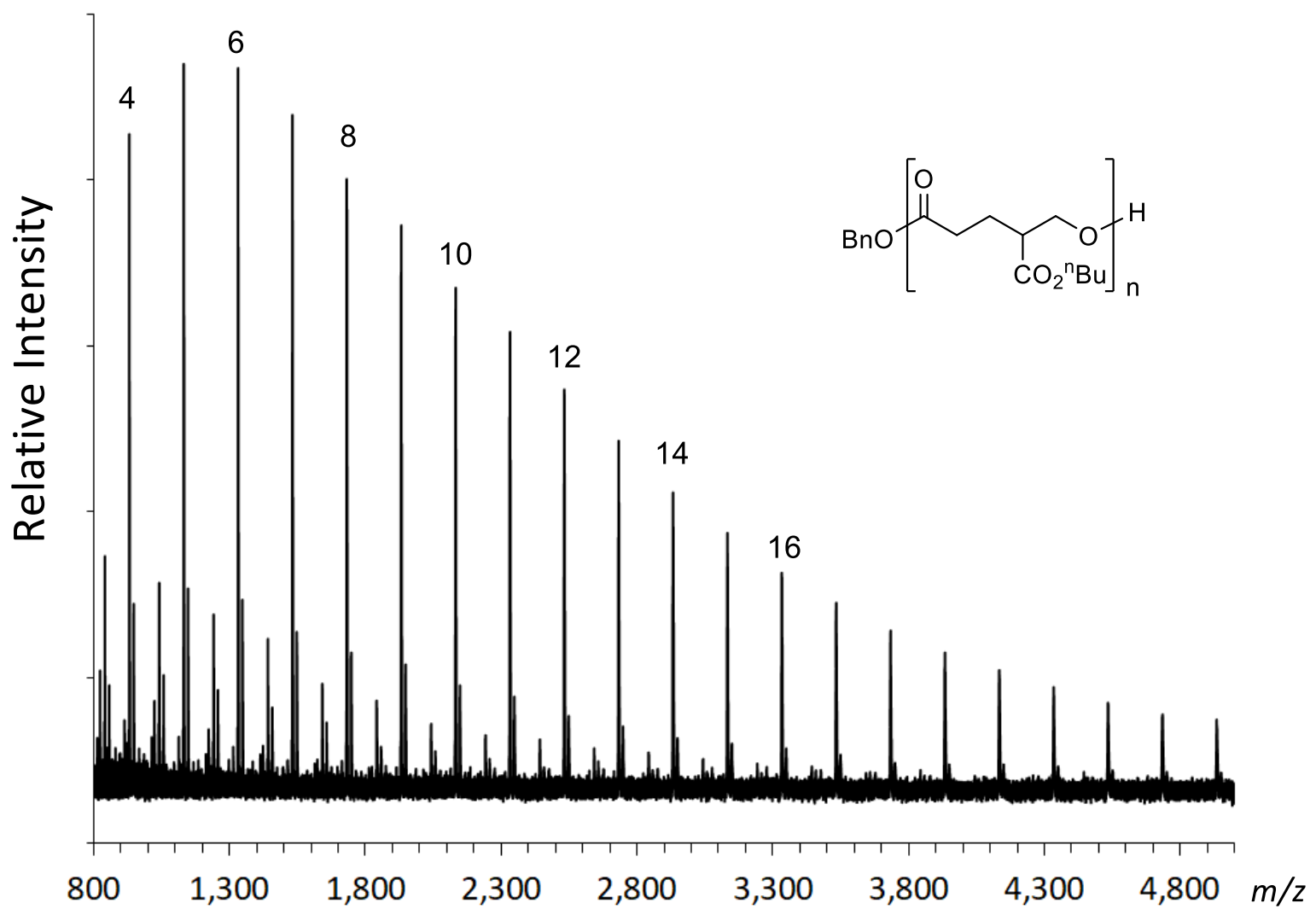

Figure S1. MALDI/TOF-MS spectrum taken in reflectron mode of PCnBuVL $\left(M_{\mathrm{n}}=3.1 \mathrm{~kg} \mathrm{~mol}^{-1}\right.$ from ${ }^{1} \mathrm{H}$ NMR spectroscopy) using dihydroxybenzene as a matrix. CnBuVL molar mass $=200 \mathrm{~g}$ $\mathrm{mol}^{-1}$. 
IVb. DBU-promoted eliminative degradation

Degradation of the PCRVLs (molecular weights of ca. $100 \mathrm{~kg} \mathrm{~mol}^{-1}$ ) was performed using an excess of 1,8-diazabicyclo[5.4.0] undec-7-ene (ca. 5 equiv, DBU) at room temperature. ${ }^{1} \mathrm{H} \mathrm{NMR}$ analysis of the resulting mixture showed only small portion of resonances indicative of branching repeat units (see below). ${ }^{10}$ We prepared another sample of PCMeVL (described below) that was polymerized for a much longer period of time. The ${ }^{1} \mathrm{H}$ NMR spectrum (Figure S2) of this aged sample of PCMeVL showed a methyl ester resonance (3.67 ppm) with a ca. 1:20 ratio to that of the methyl ester resonance for the appendages on the linear PCMeVL backbone (3.71 ppm). DBU degradation of this PCMeVL showed the presence of the 2methyleneglutarate derivatives S6, S7, and S8, which are consistent with branch subunits (Figure S3).

\section{Procedure}

CMeVL (100 equiv, $178 \mathrm{mg}, 1.13 \mathrm{mmol}$ ), $\mathrm{BnOH}$ ( 1 equiv, $1 \mu \mathrm{L}, 0.01 \mathrm{mmol}$ ), diphenyl phosphate $\left[7.6 \mathrm{mg}, 0.03 \mathrm{mmol}, \mathrm{DPP},(\mathrm{PhO})_{2} \mathrm{PO}_{2} \mathrm{H}\right]$, and a stir bar were sequentially added to a 1dram vial in a nitrogen-filled glovebox. The vial was sealed with a Teflon-lined cap, removed from the box, and placed into an oil bath at $85{ }^{\circ} \mathrm{C}$ for 4 days. The polymer was purified by precipitation as described above.

A portion of this PCMeVL $(20 \mathrm{mg})$, DBU $(200 \mu \mathrm{L})$, and a stir bar were placed in a 0.5 -dram vial and capped. The mixture was stirred for $15 \mathrm{~h}$, and an aliquot (ca. $20 \mu \mathrm{L}$ ) was dissolved in $\mathrm{CDCl}_{3}(0.55 \mathrm{~mL})$ and analyzed by ${ }^{~} \mathrm{H}$ NMR spectroscopy. 


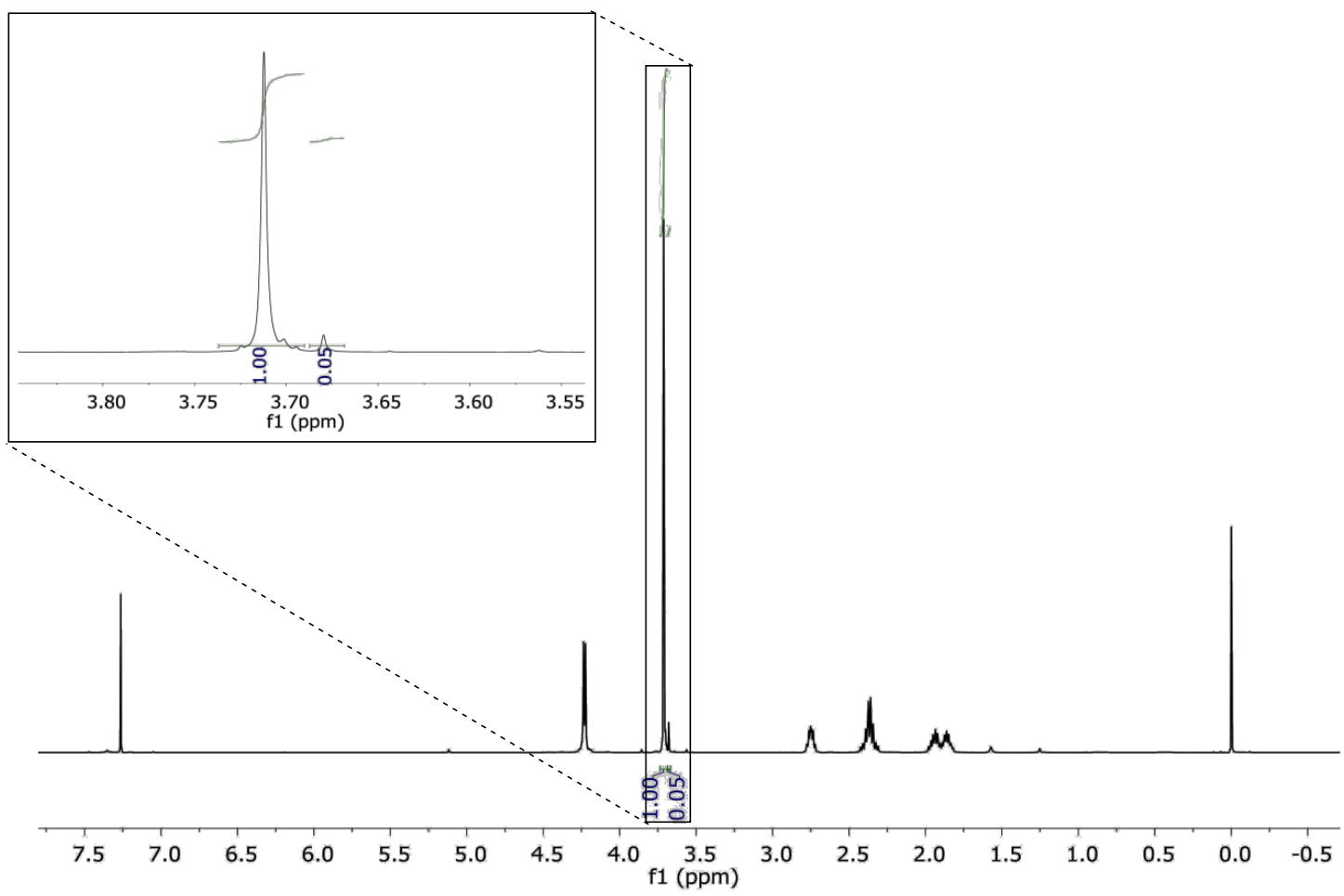

Figure S2. ${ }^{1} \mathrm{H}$ NMR spectrum of PCMeVL with ca. 100 repeat units per chain that was obtained by a polymerization carried our for 4 days at $85^{\circ} \mathrm{C}$. The inlay shows the presence of a smaller upfield methyl ester resonance, suggestive of a small amount of branching.

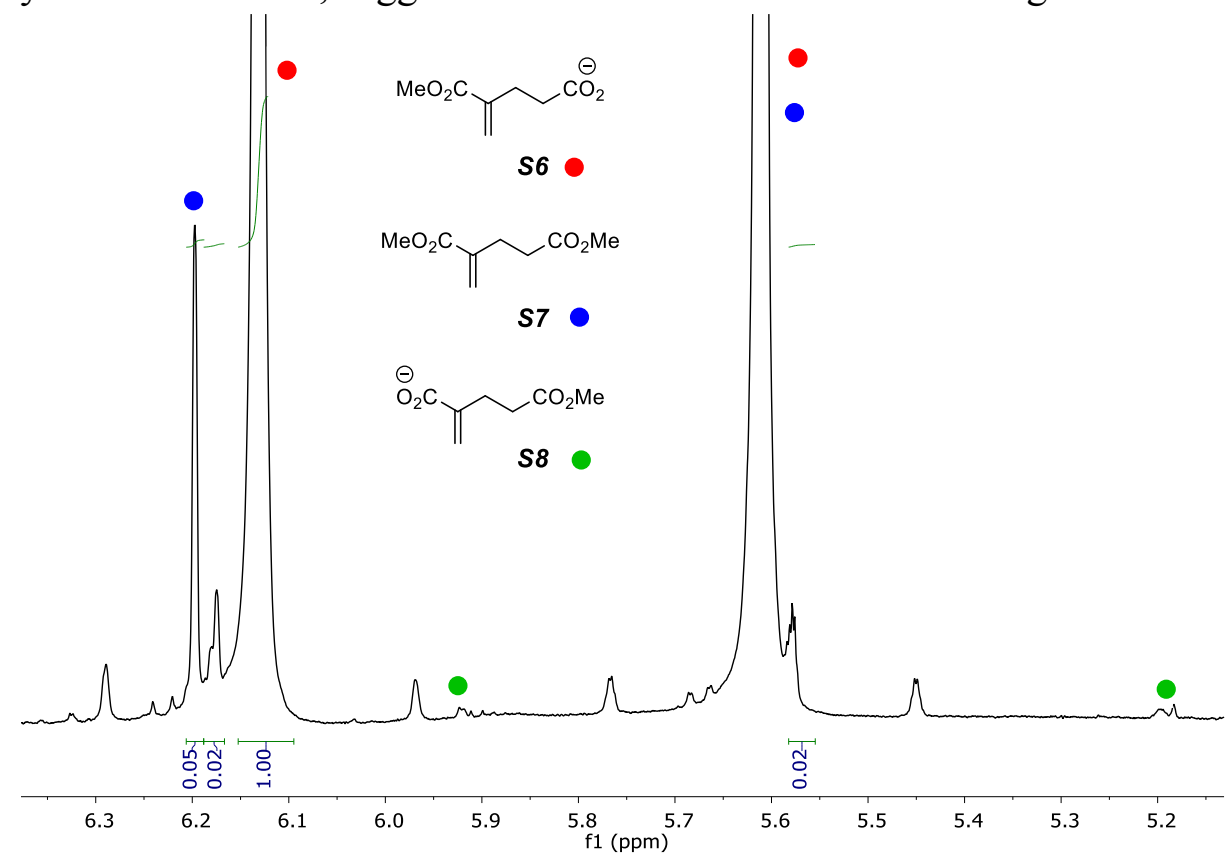

Figure S3. ${ }^{1} \mathrm{H}$ NMR spectrum of the crude degradation mixture from treating the above sample of PCMeVL with DBU. The spectrum shows the main degradation product of PCMeVL (degraded to S6) and two smaller resonance of 2-methyleneglutarates S7 and S8 that arise from transesterification reactions of the side chain methyl ester with the propagating hydroxy group. ${ }^{10}$ 
IVc. Differential scanning calorimetry (DSC) annealing studies

A sample of PCMeVL, PCEtVL, PCiPrVL, or PCnBuVL in the DSC instrument was heated to $150{ }^{\circ} \mathrm{C}\left(10{ }^{\circ} \mathrm{C} \min ^{-1}\right)$, cooled $\left(10{ }^{\circ} \mathrm{C} \mathrm{min}^{-1}\right)$ to the target temperature, and held at this temperature for $1 \mathrm{~h}$. The sample was then cooled (or warmed) to 20 or $25^{\circ} \mathrm{C}\left(10{ }^{\circ} \mathrm{C} \mathrm{min}{ }^{-1}\right)$ and then heated to $150{ }^{\circ} \mathrm{C}\left(10^{\circ} \mathrm{C} \mathrm{min}^{-1}\right)$. The sample was again cooled to a new target temperature, and this sequence was repeated to scan over a total of four or five different annealing temperatures. The subsequent heat-and-hold experiment for each polymer was chosen based on the annealing temperature that provided the largest enthalpy of melting $\left(\Delta H_{\mathrm{m}}\right)$.

DSC heating cycles were performed with varying annealing times (at the previously determined ultimate annealing temperatures) to examine the aging of the PCRVLs. PCRVL samples were heated to $150{ }^{\circ} \mathrm{C}$, cooled to and held at the determined annealing temperature for the indicated time, cooled to $20^{\circ} \mathrm{C}\left(10^{\circ} \mathrm{C} \mathrm{min}-1\right)$, and reheated to $150{ }^{\circ} \mathrm{C}\left(10^{\circ} \mathrm{C} \mathrm{min}^{-1}\right)$. The sample was again cooled to the determined annealing temperature, and this sequence was repeated for each different timeframe. 


\section{PCMeVL}

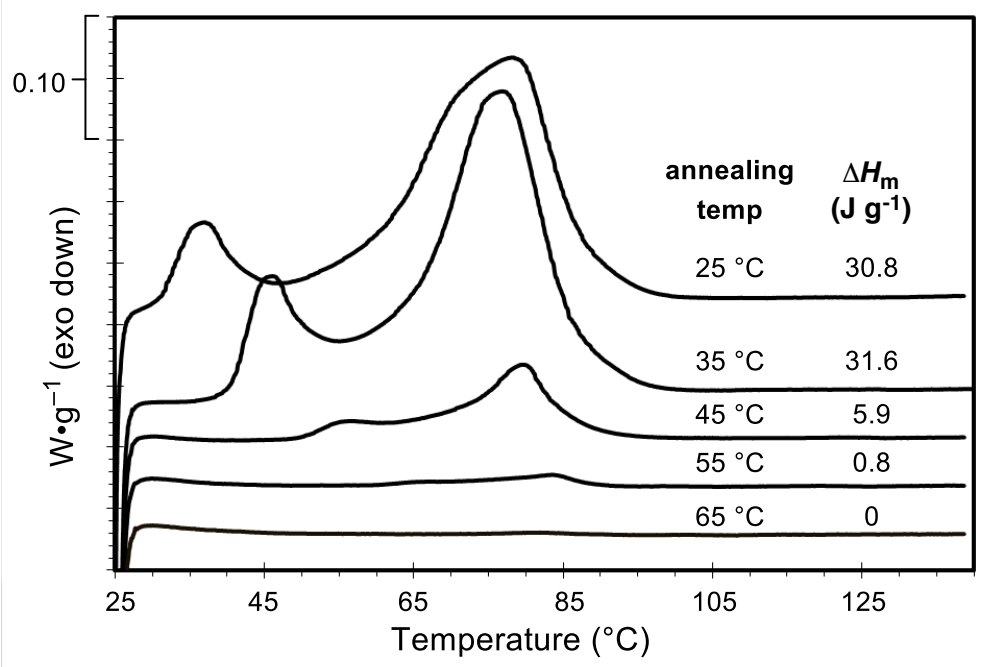

Figure S4. Annealing temperature screening. Vertically offset DSC thermograms of PCMeVL $\left[M_{\mathrm{w}}=130 \mathrm{~kg} \mathrm{~mol}^{-1}(\mathrm{MALS})\right]$ annealed for $1 \mathrm{~h}$ at $25,35,45,55$, and $65^{\circ} \mathrm{C}$. The sample was heated to $150{ }^{\circ} \mathrm{C}$, cooled to the indicated temperature $\left(10^{\circ} \mathrm{C} \mathrm{min}^{-1}\right)$, annealed for one hour, further cooled to $25^{\circ} \mathrm{C}\left(10^{\circ} \mathrm{C} \mathrm{min}^{-1}\right)$, and heated to $150{ }^{\circ} \mathrm{C}\left(10^{\circ} \mathrm{C} \mathrm{min}{ }^{-1}\right)$. The data shown are for the final heating ramp.

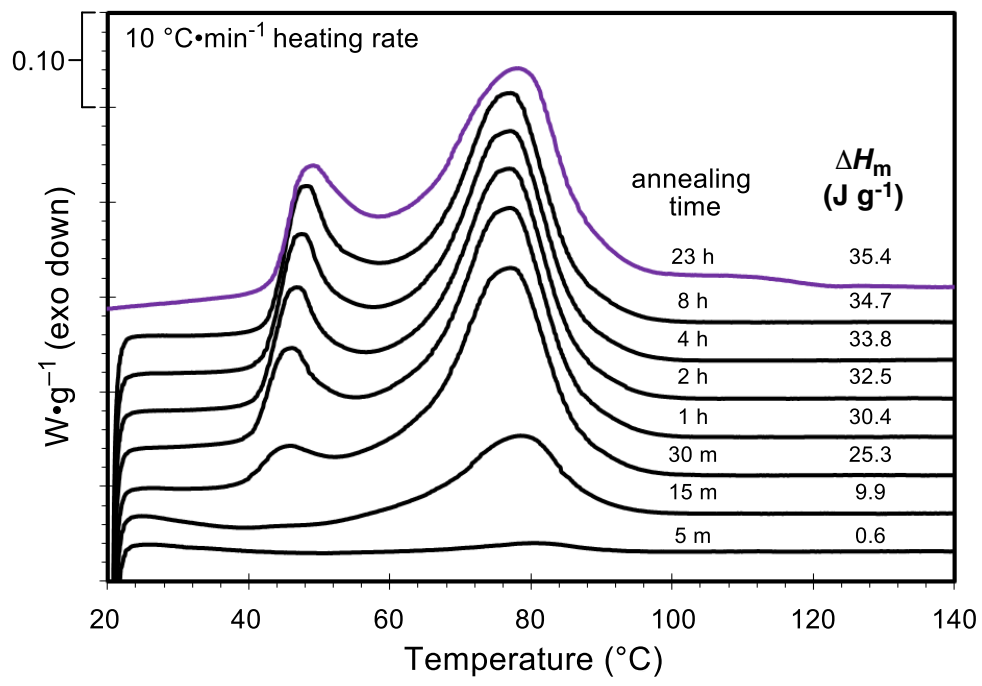

Figure S5. Annealing over time. Vertically offset DSC thermograms of PCMeVL $\left(\left[M_{\mathrm{w}}=130\right.\right.$ $\mathrm{kg} \mathrm{mol}^{-1}$ (MALS)]) annealed at $35^{\circ} \mathrm{C}$ for indicated time lengths. The sample was heated to 150 ${ }^{\circ} \mathrm{C}$, cooled to $35^{\circ} \mathrm{C}$ (the ultimate annealing temperature for PCMeVL; $10{ }^{\circ} \mathrm{C} \mathrm{min}^{-1}$ ), annealed for the indicated time, further cooled to $20^{\circ} \mathrm{C}\left(10^{\circ} \mathrm{C} \min ^{-1}\right)$, and heated to $150{ }^{\circ} \mathrm{C}\left(10^{\circ} \mathrm{C} \mathrm{min}^{-1}\right)$. The analysis was repeated for each new annealing time. The 23-h timepoint was recorded for a sample that had been melt pressed and annealed at $35^{\circ} \mathrm{C}$ for $23 \mathrm{~h}$. This sample was subsequently used for tensile testing. Each trace was integrated from $41-100{ }^{\circ} \mathrm{C}$ to determine $\Delta H_{\mathrm{m}}$. 


\section{PCEtVL}

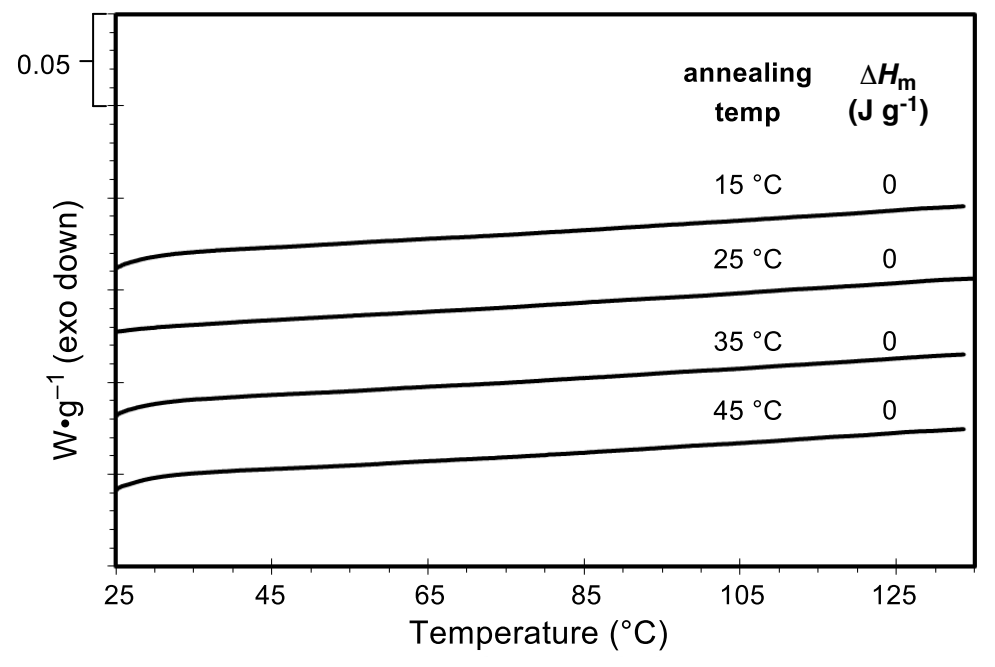

Figure S6. Annealing temperature screening. Vertically offset DSC thermograms of PCEtVL $\left[M_{\mathrm{w}}=86 \mathrm{~kg} \mathrm{~mol}^{-1}(\mathrm{MALS})\right]$ annealed for $1 \mathrm{~h}$ at $25,35,45,55$, and $65^{\circ} \mathrm{C}$. The sample was heated to $150{ }^{\circ} \mathrm{C}$, cooled to the indicated temperature $\left(10^{\circ} \mathrm{C} \mathrm{min}{ }^{-1}\right)$, annealed for one hour, further cooled to $25^{\circ} \mathrm{C}\left(10^{\circ} \mathrm{C} \mathrm{min}{ }^{-1}\right)$, and heated to $150{ }^{\circ} \mathrm{C}\left(10^{\circ} \mathrm{C} \mathrm{min}{ }^{-1}\right)$. The data shown are for the final heating ramp.

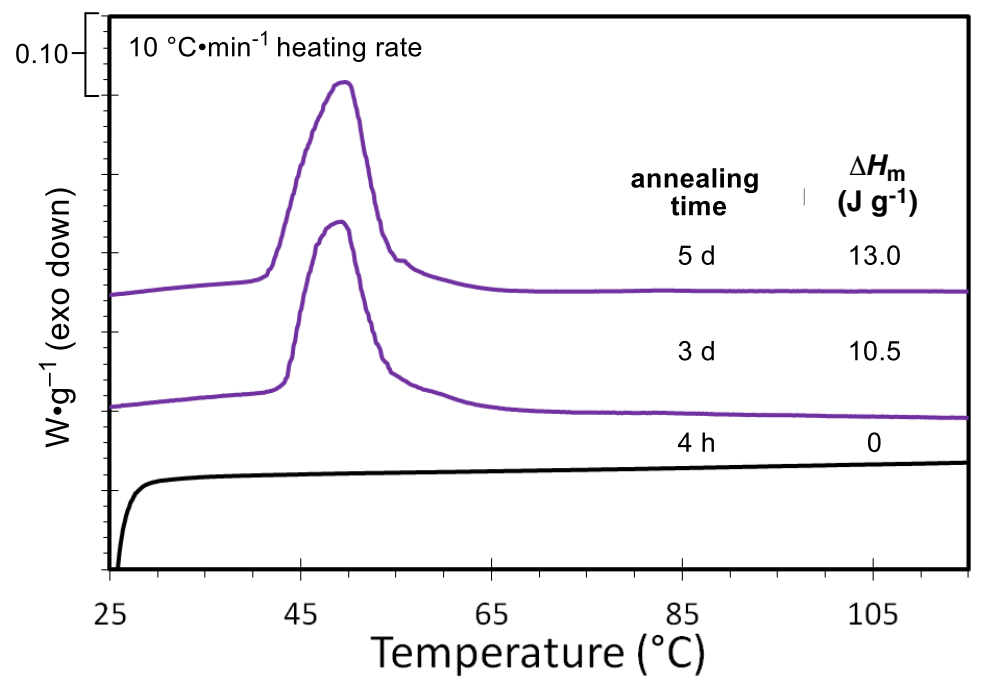

Figure S7. Annealing over time. Vertically offset DSC thermograms of PCEtVL $\left[M_{\mathrm{w}}=86 \mathrm{~kg}\right.$ $\mathrm{mol}^{-1}$ (MALS)] annealed at RT (ca. $22^{\circ} \mathrm{C}$ ) for the indicated lengths of time. The $4 \mathrm{~h}$ data were collected for a sample that was heated to $150{ }^{\circ} \mathrm{C}$, cooled to $25^{\circ} \mathrm{C}\left(10^{\circ} \mathrm{C} \mathrm{min}{ }^{-1}\right)$, annealed for four hours, and heated to $150{ }^{\circ} \mathrm{C}\left(10^{\circ} \mathrm{C} \mathrm{min}^{-1}\right)$. The other two data sets were collected for samples that had been melt pressed and annealed at RT $\left(22^{\circ} \mathrm{C}\right)$ for 3 and 5 days, respectively. Each trace was integrated from $39-65^{\circ} \mathrm{C}$ to determine $\Delta H_{\mathrm{m}}$. 


\section{PCiPrVL}

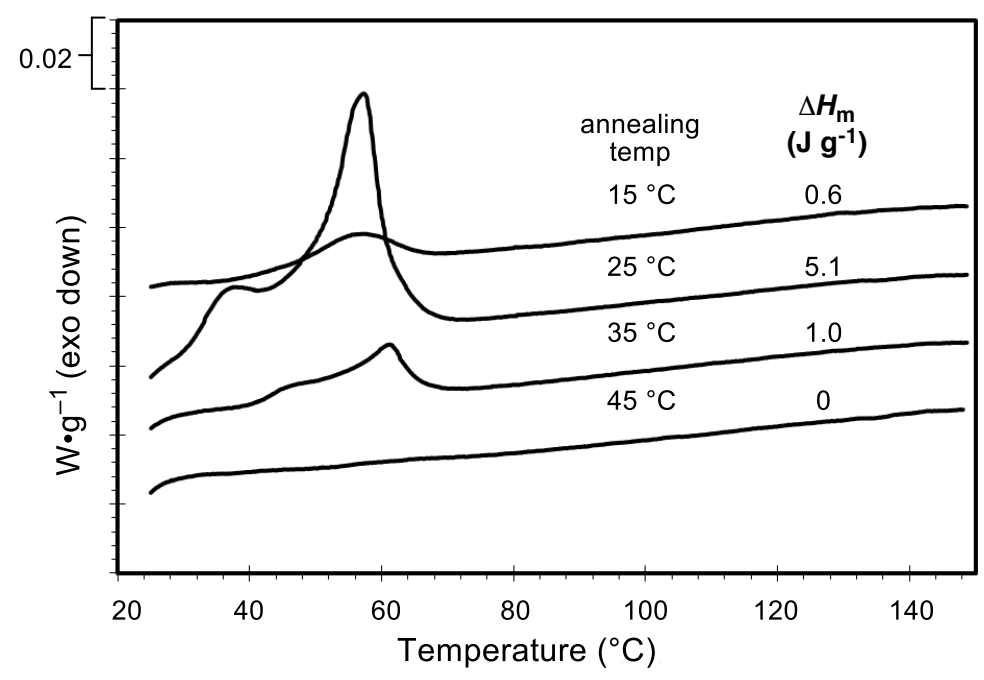

Figure S8. Annealing temperature screening. Vertically offset DSC thermograms of PCiPrVL $\left[M_{\mathrm{w}}=65 \mathrm{~kg} \mathrm{~mol}^{-1}(\mathrm{MALS})\right]$ annealed for $1 \mathrm{~h}$ at $15,25,35$, and $45^{\circ} \mathrm{C}$. The sample was heated to $150{ }^{\circ} \mathrm{C}$, cooled to the indicated temperature $\left(10^{\circ} \mathrm{C} \mathrm{min}^{-1}\right)$, annealed for one hour, further cooled (or heated) to $25^{\circ} \mathrm{C}\left(10^{\circ} \mathrm{C} \mathrm{min}^{-1}\right)$, and heated to $150{ }^{\circ} \mathrm{C}\left(10^{\circ} \mathrm{C} \mathrm{min}^{-1}\right)$. The data shown are for the final heating ramp at each annealing temperature.

The data for scans taken at the ultimate annealing temperature $\left(22^{\circ} \mathrm{C}\right)$ for varying lengths of time are shown in Figure $7 \mathrm{~b}$ in the manuscript. 


\section{PCnBuVL}

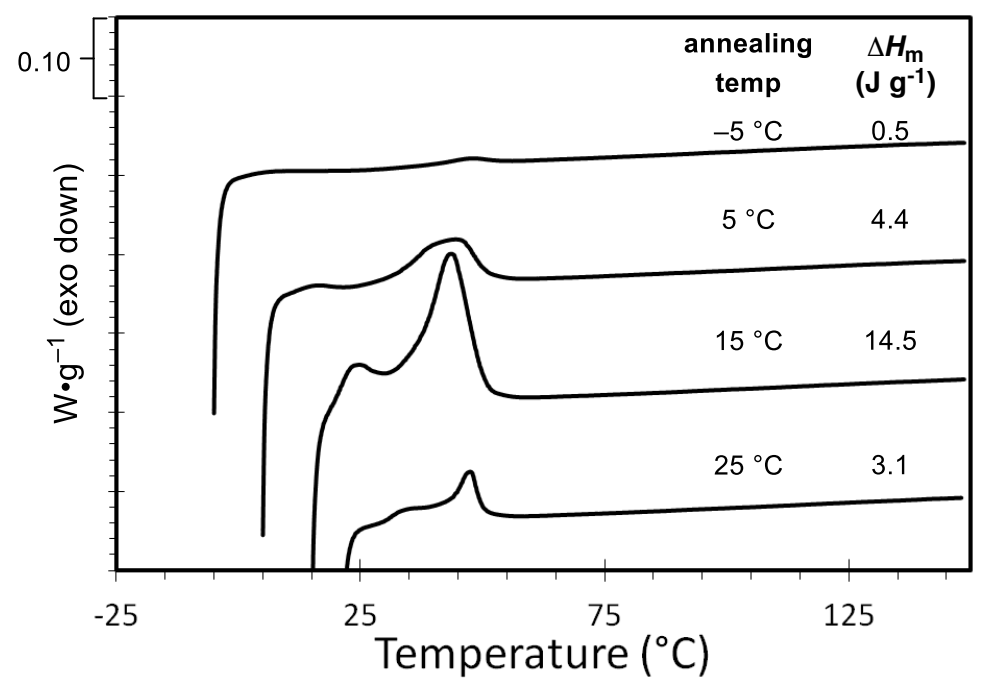

Figure S9. Annealing temperature screening. Vertically offset DSC thermograms of PCnBuVL $\left[M_{\mathrm{w}}=90 \mathrm{~kg} \mathrm{~mol}^{-1}\right.$ (MALS) $]$ annealed for $1 \mathrm{~h}$ at $-5,5,15$, and $25^{\circ} \mathrm{C}$. The sample was heated to $150{ }^{\circ} \mathrm{C}$, cooled to the indicated temperature $\left(10^{\circ} \mathrm{C} \mathrm{min}^{-1}\right)$, annealed for one hour, further cooled to $25^{\circ} \mathrm{C}\left(10^{\circ} \mathrm{C} \mathrm{m^{-1 }}\right)$, and heated to $150{ }^{\circ} \mathrm{C}\left(10{ }^{\circ} \mathrm{C} \mathrm{min}{ }^{-1}\right)$. The data shown are for the final heating ramp at each of the annealing temperatures.

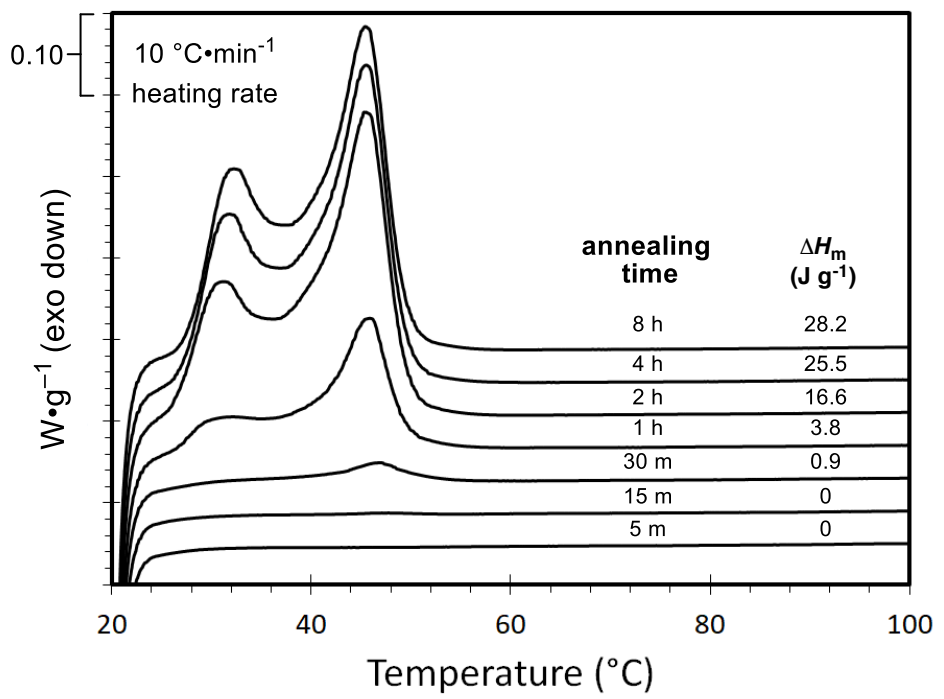

Figure S10. Annealing over time. Vertically offset DSC thermograms of PCnBuVL $\left[M_{\mathrm{w}}=90\right.$ $\mathrm{kg} \mathrm{mol}^{-1}$ (MALS)] annealed at RT (ca. $22^{\circ} \mathrm{C}$ ) for the indicated lengths of time. Each sample was heated to $150{ }^{\circ} \mathrm{C}$, cooled to $22{ }^{\circ} \mathrm{C}\left(10{ }^{\circ} \mathrm{C} \mathrm{min}{ }^{-1}\right)$, annealed, and heated to $150{ }^{\circ} \mathrm{C}\left(10{ }^{\circ} \mathrm{C} \mathrm{min}\right.$ $\left.{ }^{1}\right)$. Each trace was integrated from $25-65^{\circ} \mathrm{C}$ to determine $\Delta H_{\mathrm{m}}$. 


\section{Polymer Characterization Data}

Va. Size-exclusion chromatography

a) PCMeVL

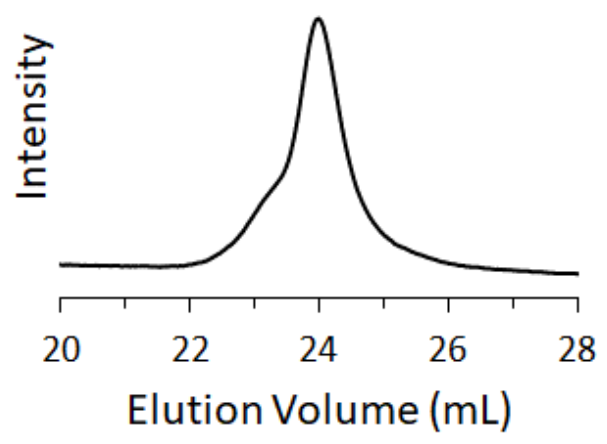

$M_{\mathrm{n}}=115.6 \mathrm{~kg} \mathrm{~mol}^{-1}, M_{\mathrm{w}}=129.9 \mathrm{~kg} \mathrm{~mol}^{-1}$, $Ð=1.12$

c) PCiPrVL

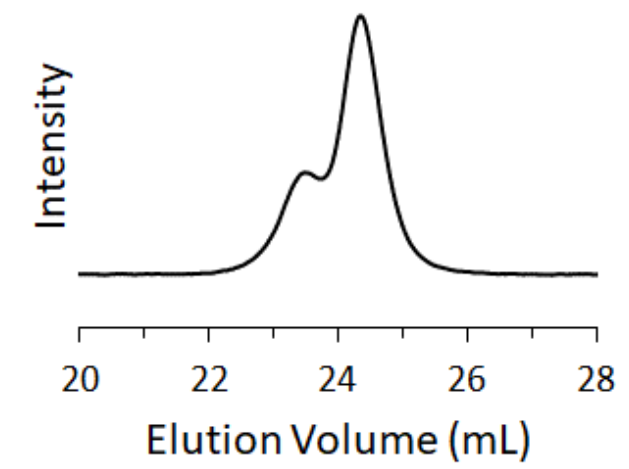

$$
M_{\mathrm{n}}=56.6 \mathrm{~kg} \mathrm{~mol}^{-1}, M_{\mathrm{w}}=64.8 \mathrm{~kg} \mathrm{~mol}^{-1} \text {, }
$$$$
Ð=1.15
$$

b) PCEtVL

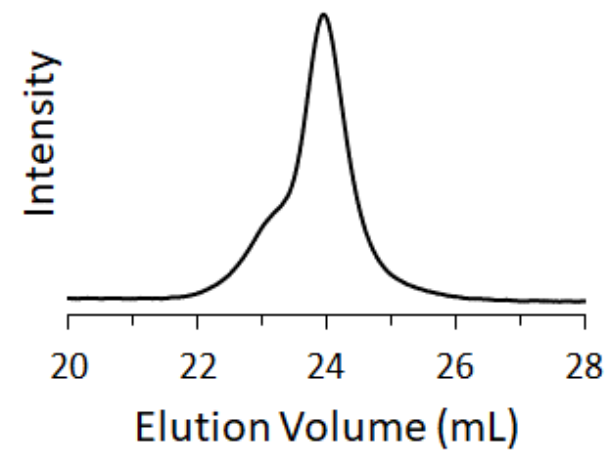

$M_{\mathrm{n}}=76.1 \mathrm{~kg} \mathrm{~mol}^{-1}, M_{\mathrm{w}}=86.3 \mathrm{~kg} \mathrm{~mol}^{-1}$, $Ð=1.14$

d) PCnBuVL

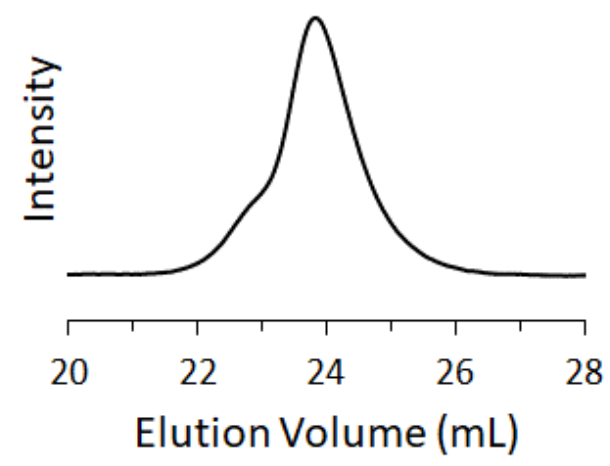

$M_{\mathrm{n}}=75.0 \mathrm{~kg} \mathrm{~mol}^{-1}, M_{\mathrm{w}}=90.3 \mathrm{~kg} \mathrm{~mol}^{-1}$, $Ð=1.21$

Figure S11. SEC chromatograms of PCRVLs with target $M_{\mathrm{n}}$ of $110 \mathrm{~kg} \mathrm{~mol}^{-1}$. Each sample was polymerized at $70{ }^{\circ} \mathrm{C}$ to ca. $90 \%$ conversion and purified through precipitation. Samples were analyzed using a THF-SEC fitted with a MALS detector. 
a) PCEtVL

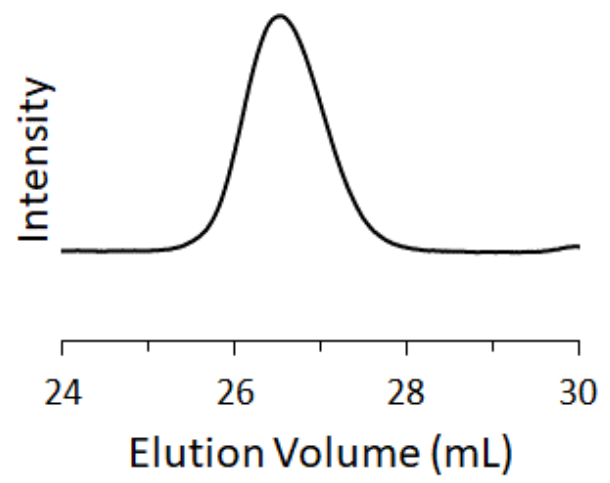

$M_{\mathrm{n}}=14.5 \mathrm{~kg} \mathrm{~mol}^{-1}, M_{\mathrm{w}}=15.5 \mathrm{~kg} \mathrm{~mol}^{-1}$,
$Ð=1.06$

c) PCnBuVL

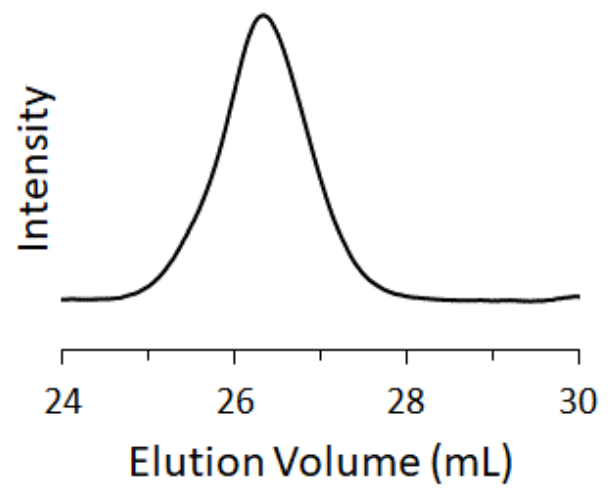

$M_{\mathrm{n}}=19.4 \mathrm{~kg} \mathrm{~mol}^{-1}, M_{\mathrm{w}}=28.3 \mathrm{~kg} \mathrm{~mol}^{-1}$,
$Ð=1.46$ b) PCiPrVL

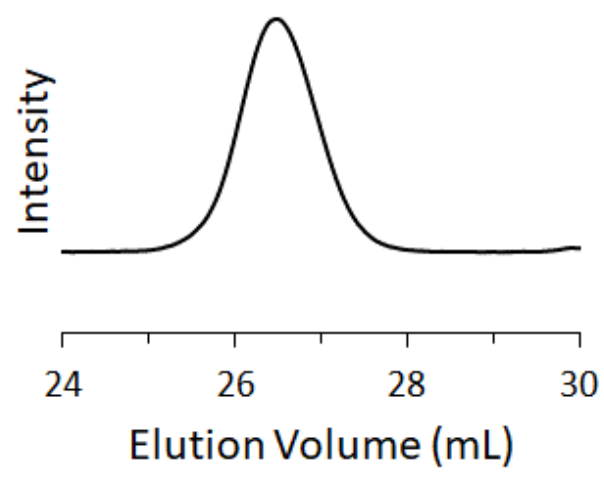

$M_{\mathrm{n}}=14.9 \mathrm{~kg} \mathrm{~mol}^{-1}, M_{\mathrm{w}}=15.4 \mathrm{~kg} \mathrm{~mol}^{-1}$, $Ð=1.03$

d) PCtBuVL

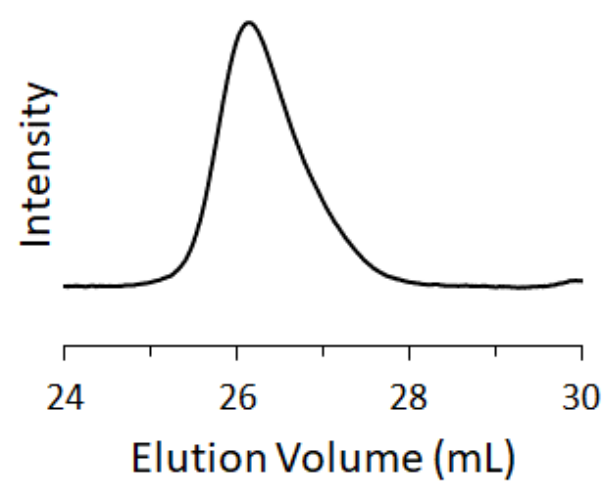
$M_{\mathrm{n}}=15.6 \mathrm{~kg} \mathrm{~mol}^{-1}, M_{\mathrm{w}}=18.0 \mathrm{~kg} \mathrm{~mol}^{-1}$,
$Đ=1.15$

Figure S12. SEC chromatograms of PCRVLs targeted to have a number average repeat unit of 50. Each sample was polymerized at room temperature and precipitated. Samples were analyzed using a THF-SEC fitted with a MALS detector. 
e) PCBnVL

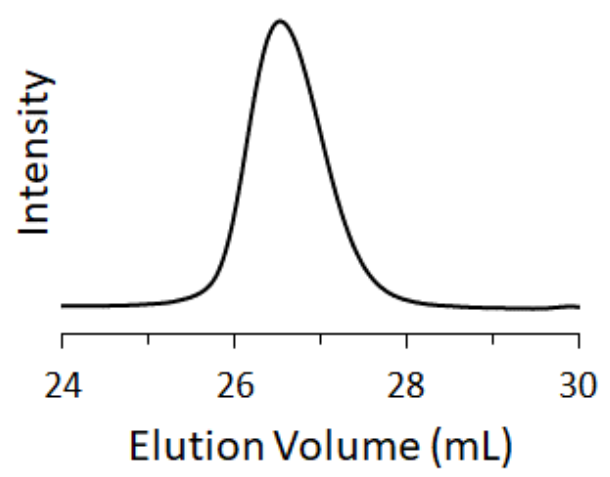

$M_{\mathrm{n}}=10.3 \mathrm{~kg} \mathrm{~mol}^{-1}, M_{\mathrm{w}}=10.9 \mathrm{~kg} \mathrm{~mol}^{-1}$, $Ð=1.05$

\section{g) $\mathrm{PCC}_{11} \mathrm{VL}$}

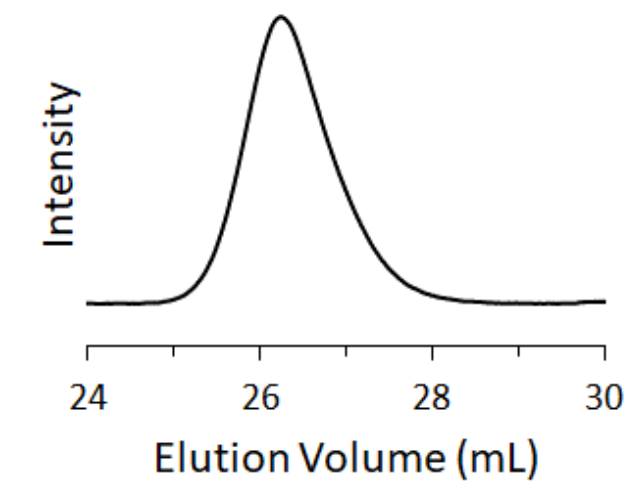

$$
\begin{gathered}
M_{\mathrm{n}}=13.2 \mathrm{~kg} \mathrm{~mol}^{-1}, M_{\mathrm{w}}=16.4 \mathrm{~kg} \mathrm{~mol}^{-1}, \\
Ð=1.18
\end{gathered}
$$

Figure S12 (continued). SEC chromatograms of PCRVLs targeted to have a number average repeat unit of 50. Each sample was polymerized at room temperature and precipitated. Samples were analyzed using a MALS detector.

\section{f) PC $^{2}$ EtHexVL}

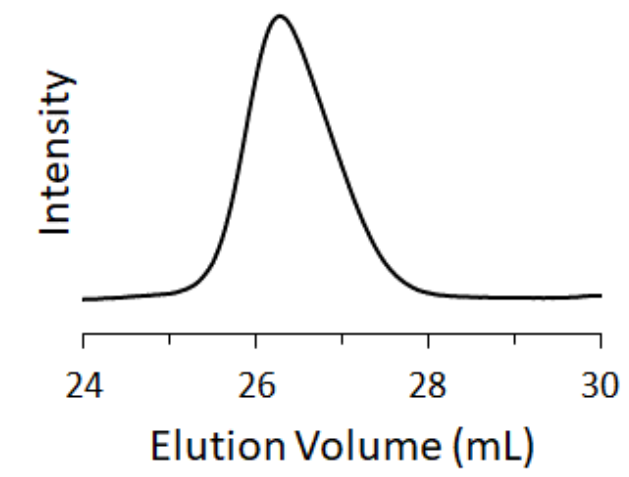

$M_{\mathrm{n}}=16.0 \mathrm{~kg} \mathrm{~mol}^{-1}, M_{\mathrm{w}}=18.6 \mathrm{~kg} \mathrm{~mol}^{-1}$, $Đ=1.21$ 
Vb. Thermogravimetric analysis (TGA)

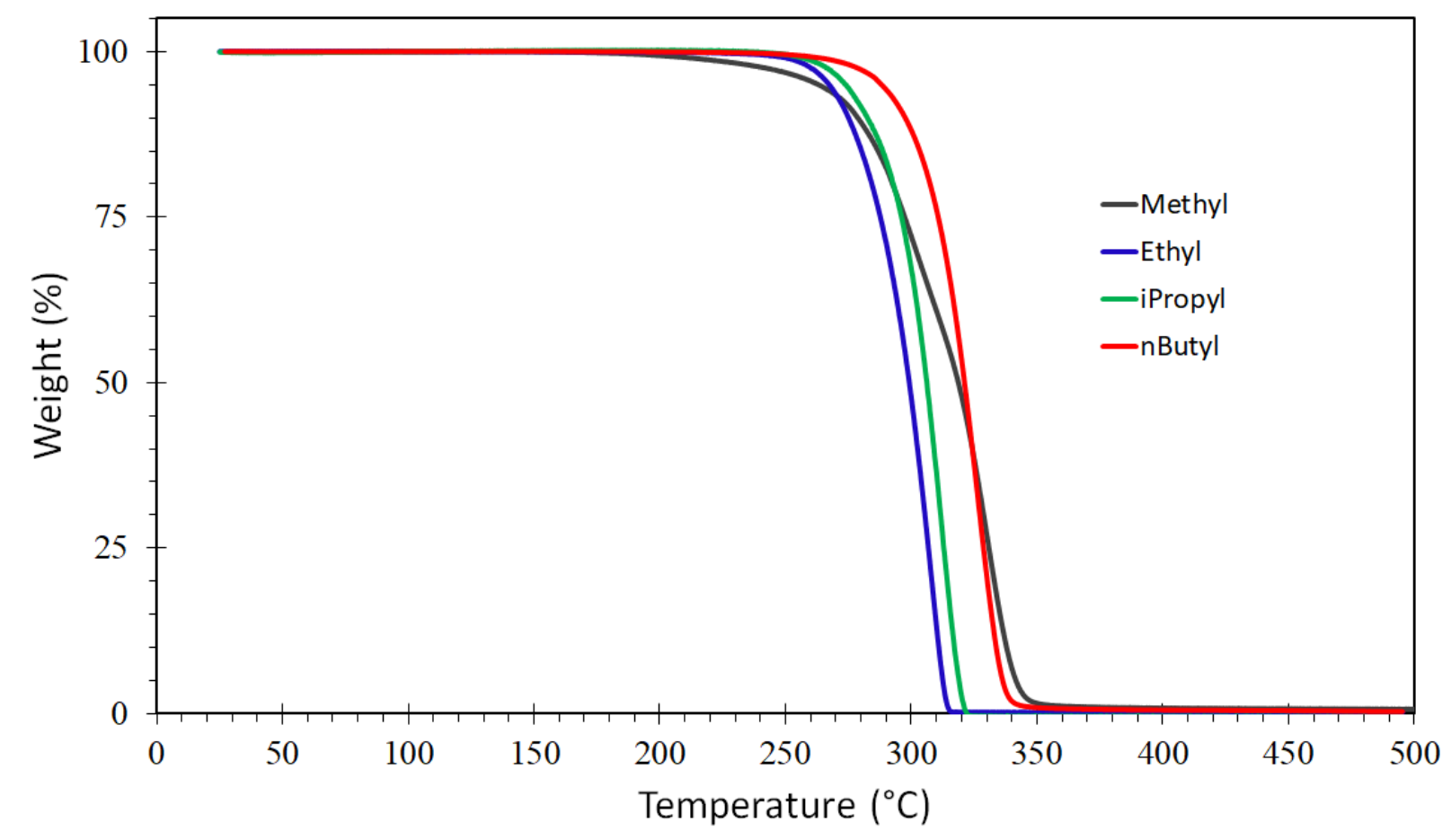

Figure S13. Overlayed TGA thermograms of high molecular weight PCRVLs $\left[M_{\mathrm{w}}=65-130 \mathrm{~kg}\right.$ $\mathrm{mol}^{-1}$ (MALS)].

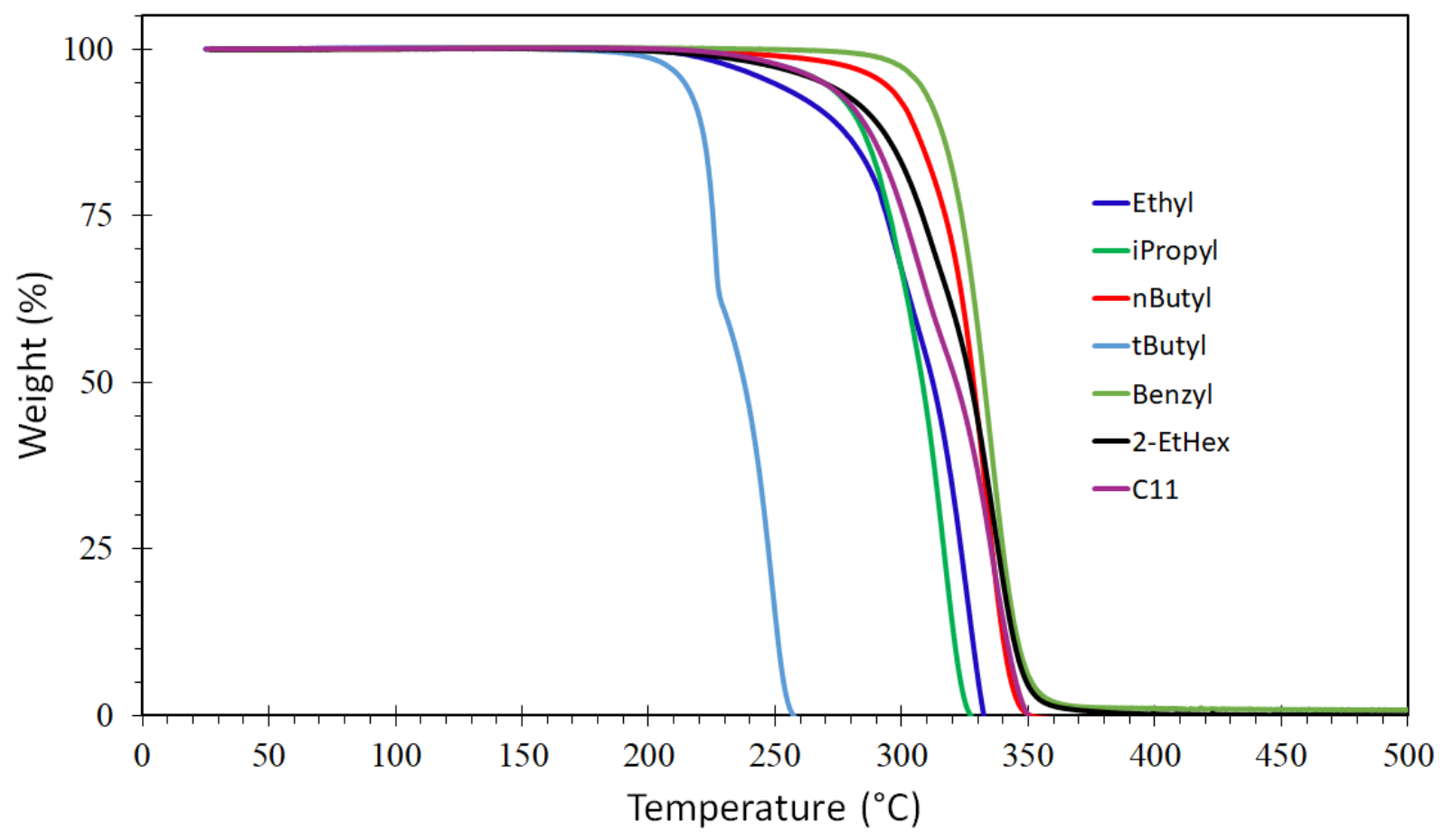

Figure S14. Overlayed TGA thermograms of PCRVLs targeted to have a number average repeat unit of 50 . 


\section{a) PCEtVL}

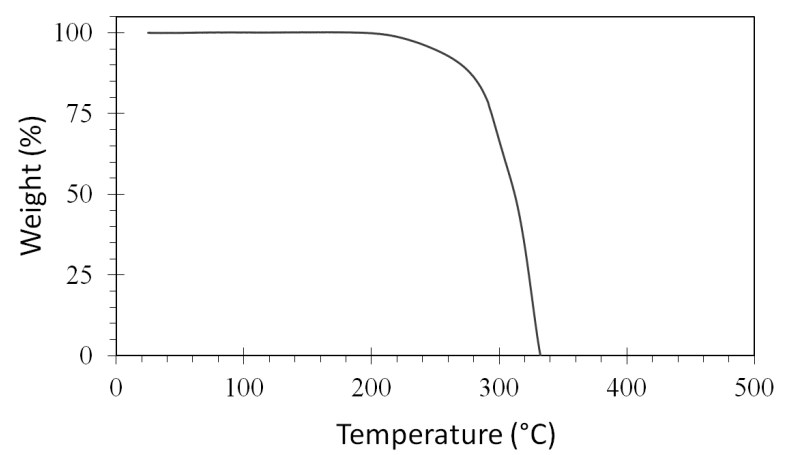

c) PCnBuVL

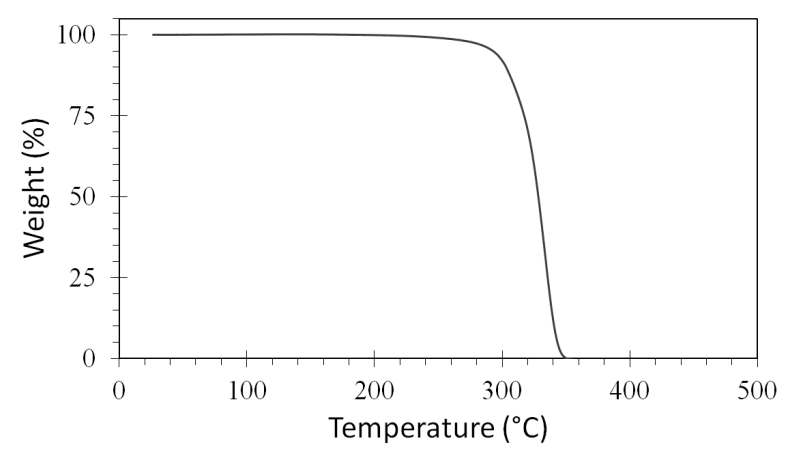

e) PCBnVL

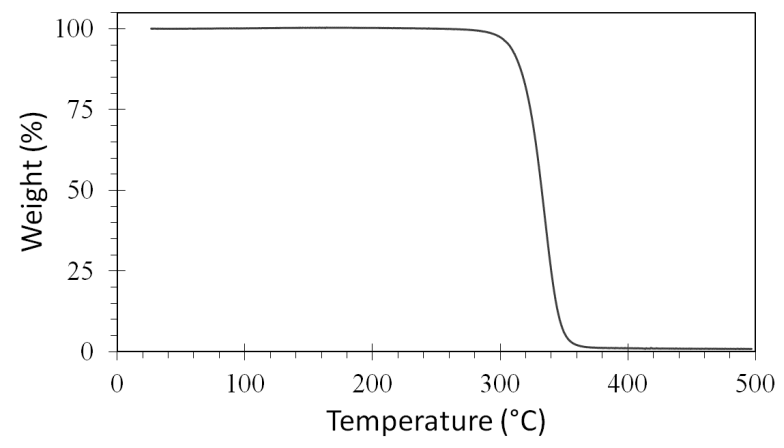

g) $\mathrm{PCC}_{11} \mathrm{VL}$

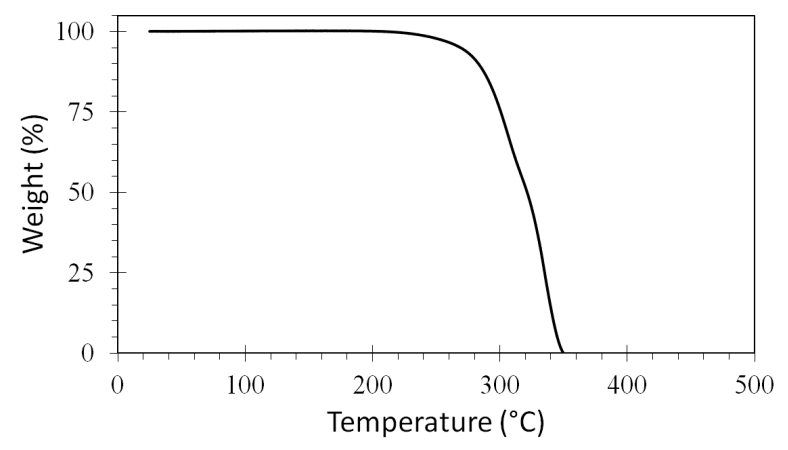

b) PCiPrVL

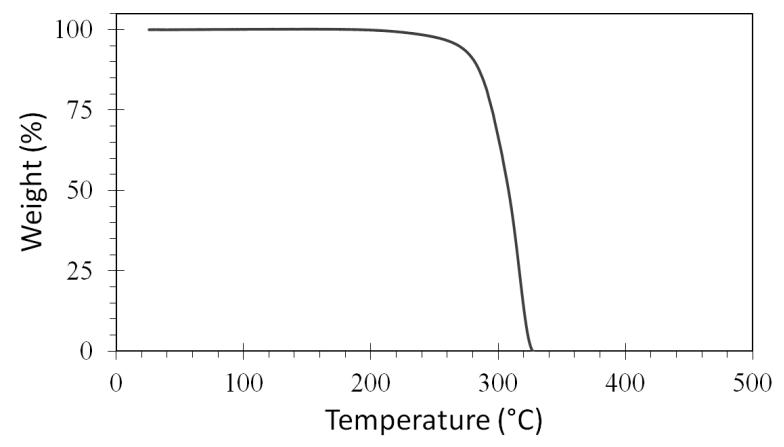

d) PCtBuVL

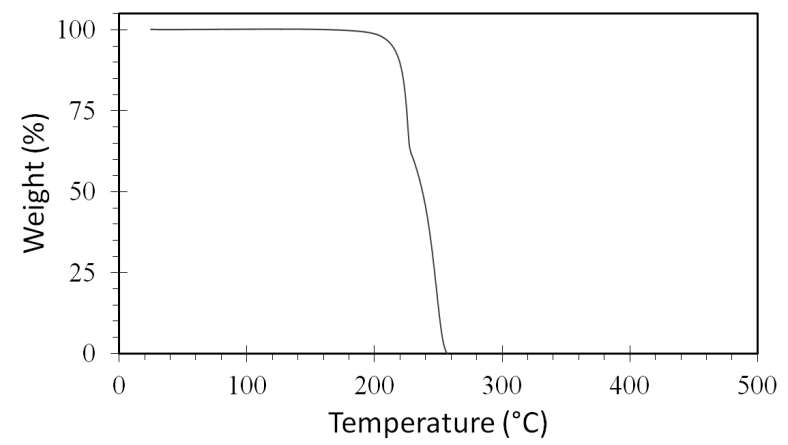

f) PC PtHexVL $^{2}$

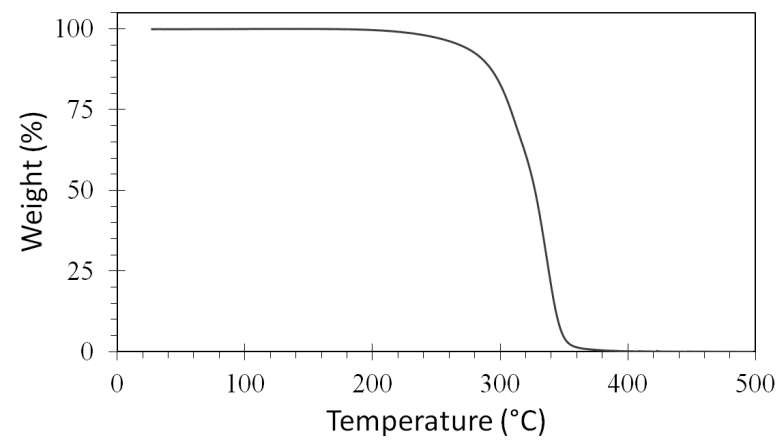

h) Summary of TGA results of PCRVLs with $n=$ ca. 50 .

\begin{tabular}{|cccc} 
& $T_{d}$ & & $T_{d}$ \\
& $\left(1 \%, 5 \%{ }^{\circ} \mathrm{C}\right)$ & & $\left(1 \%, 5 \%{ }^{\circ} \mathrm{C}\right)$ \\
\hline PCMeVL & 213,263 & PCtBuVL & 197,214 \\
PCEtVL & 218,249 & PCBnVL & 289,307 \\
PCiPrVL & 228,269 & PC $^{2}$ EtHexVL & 226,269 \\
PCnBuVL & 252,292 & PC $_{11} V L$ & 236,268 \\
\hline
\end{tabular}

Figure S15. Individual TGA thermograms of PCRVLs targeted to have a number average repeat unit of 50 (same data as Figure S14 above). Samples were heated under nitrogen at a heating rate

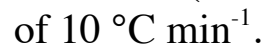


Vc. Differential scanning calorimetry

a) PCMeVL

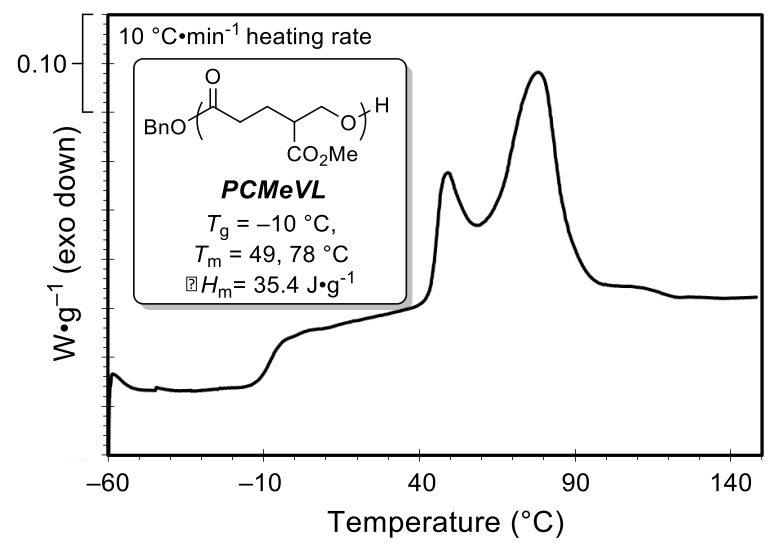

c) PCiPrVL

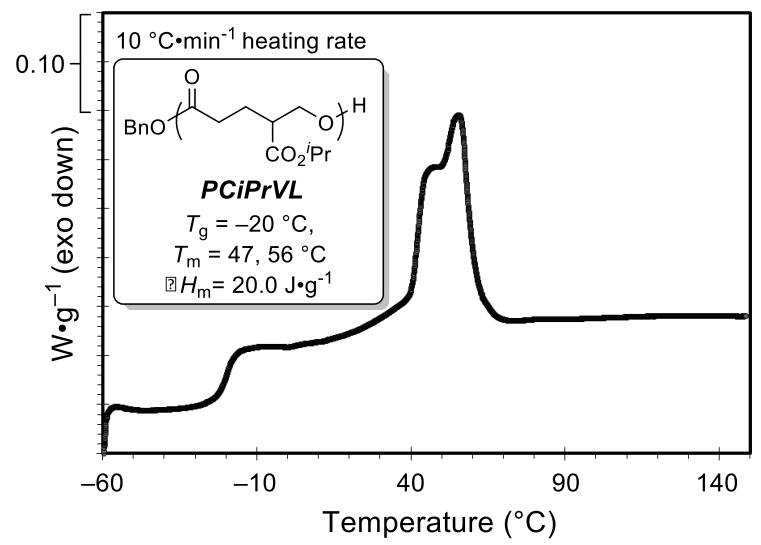

b) PCEtVL

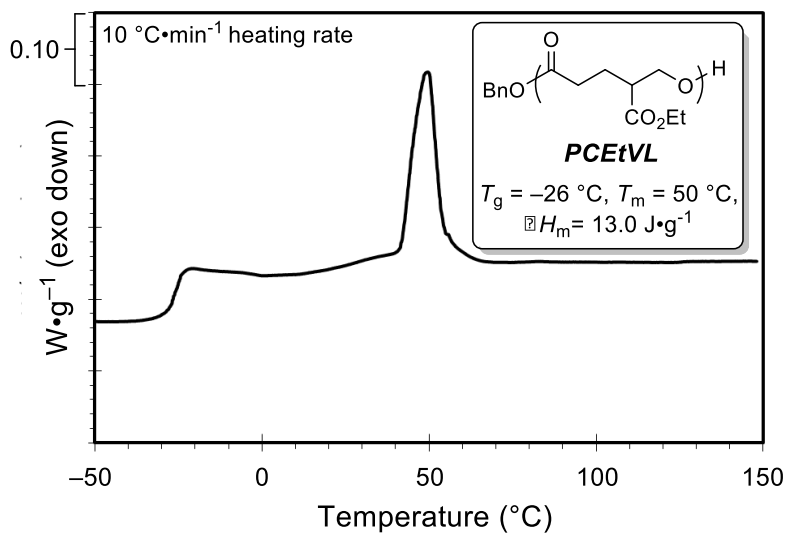

d) PCnBuVL

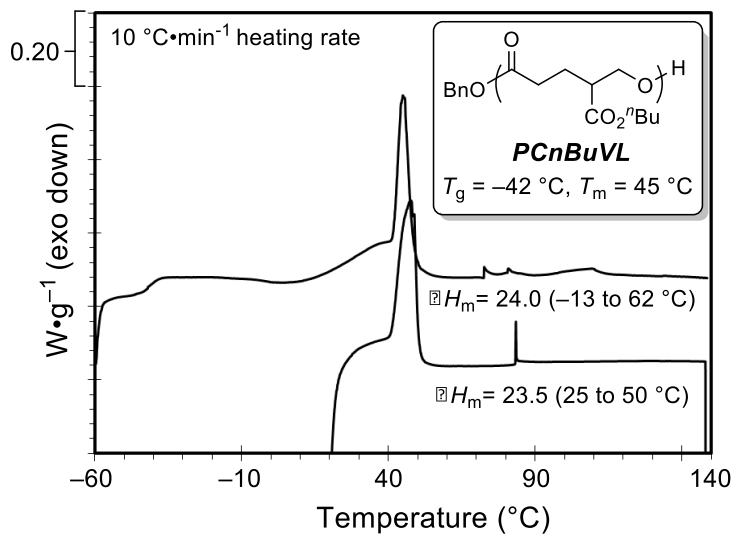

Figure S16. DSC thermograms of annealed PCRVLs prior to tensile testing $\left[M_{\mathrm{w}}=65-130 \mathrm{~kg}\right.$ $\mathrm{mol}^{-1}$ (MALS)]. DSC traces shown are taken from the first heating cycle. Each sample was annealed for a minimum of 72 hours (at ambient temperature or, for PCMeVL, at $35^{\circ} \mathrm{C}$ ). A portion of this sample was then used of the DSC measurement: cooled to $-60{ }^{\circ} \mathrm{C}$ and then heated to $150{ }^{\circ} \mathrm{C}$ at a heating rate of $10{ }^{\circ} \mathrm{C} \mathrm{min}^{-1}$. Due to the broadening of the melting exotherm of $\mathrm{PCnBuVL}$ while cooling to $-60^{\circ} \mathrm{C}$, another DSC run was performed where the sample was cooled only to $20^{\circ} \mathrm{C}$ prior to heating to $150{ }^{\circ} \mathrm{C}$. DSC measurements taken at longer annealing times showed minimal aging effects in the DSC behavior of each polymer. 
a) PCEtVL

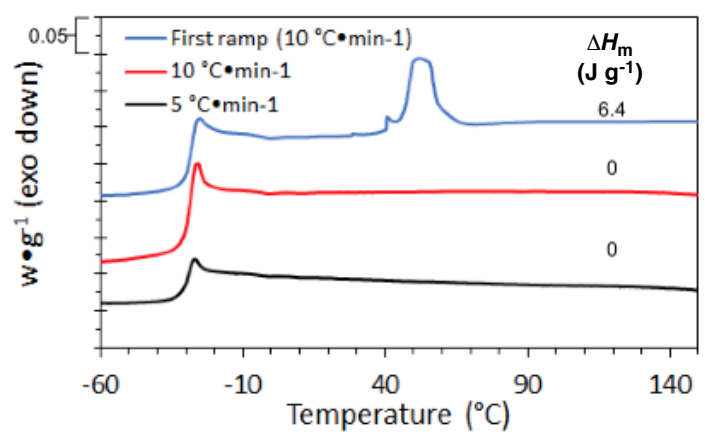

c) PCnBuVL

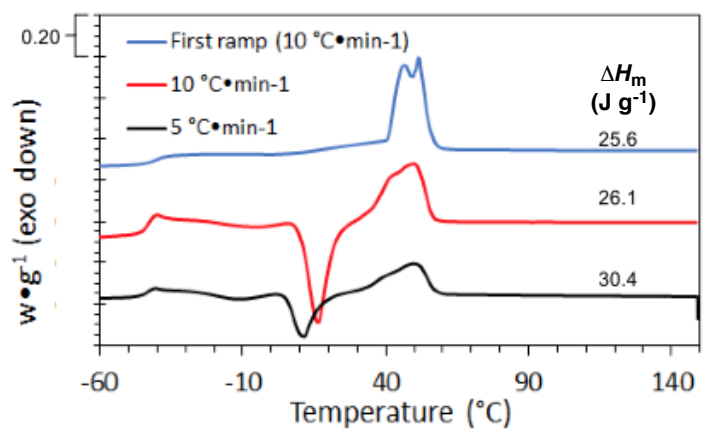

e) PCBnVL

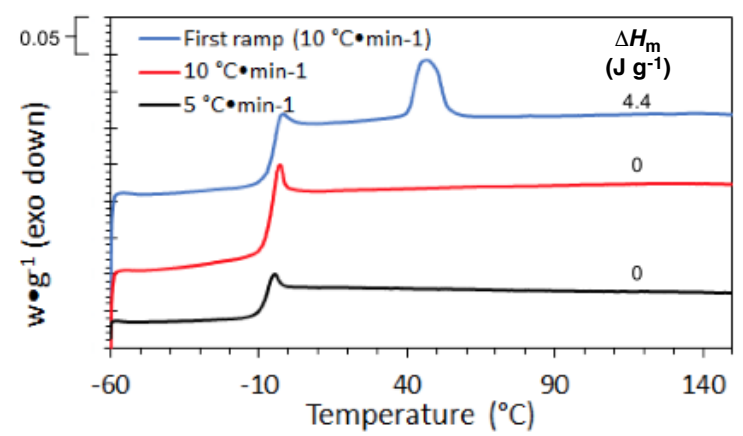

\section{g) $\mathrm{PCC}_{11} \mathrm{VL}$}

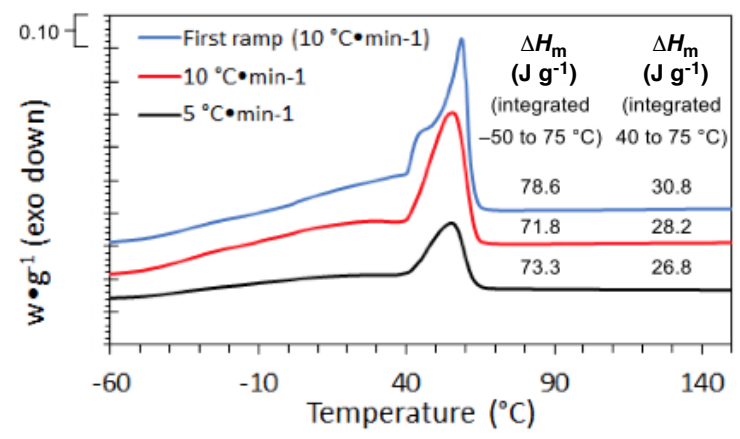

b) PCiPrVL

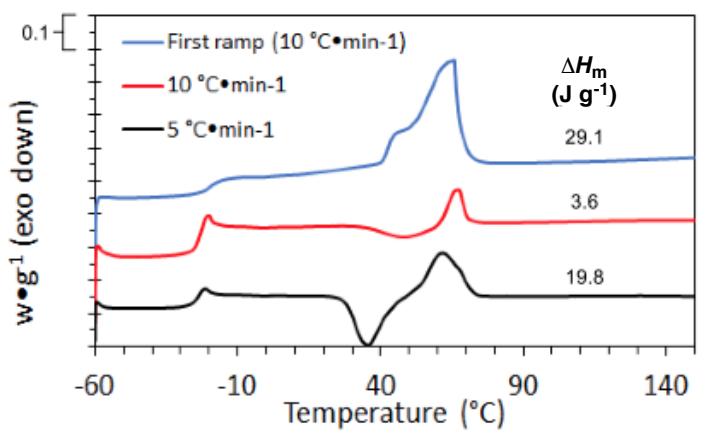

d) PCtBuVL

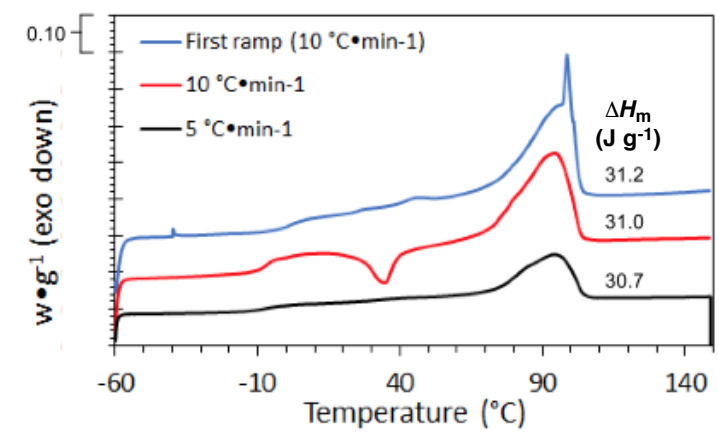

f) PC ${ }^{2}$ EtHexVL

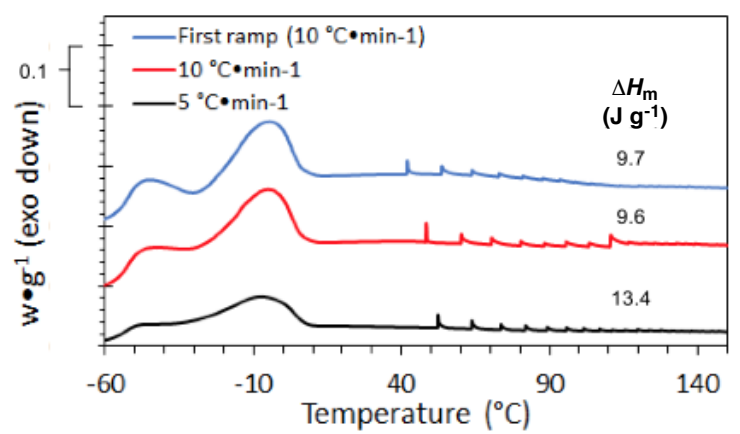

Figure S17. DSC thermograms of PCRVLs with number average of ca. 50 repeat units. DSC heating procedure: Samples were first cooled to $-60{ }^{\circ} \mathrm{C}$, heated to $175^{\circ} \mathrm{C}$ at a rate of $10^{\circ} \mathrm{C} \mathrm{min}^{-1}$ (labeled as "first ramp", blue), cooled to $-60{ }^{\circ} \mathrm{C}$ at a rate of $10^{\circ} \mathrm{C} \min ^{-1}$, reheated to $175^{\circ} \mathrm{C}$ at a heating rate of $10{ }^{\circ} \mathrm{C} \min ^{-1}$ (labeled as " $10{ }^{\circ} \mathrm{C} \min ^{-1} "$, red), cooled to $-60{ }^{\circ} \mathrm{C}$ at a rate of $5{ }^{\circ} \mathrm{C}$ $\min ^{-1}$, and finally reheated to $175^{\circ} \mathrm{C}$ at a rate of $5^{\circ} \mathrm{C} \min ^{-1}$ (labeled as " ${ }^{\circ} \mathrm{C} \mathrm{min}^{-1}$ ", black). 
Vd. Tensile testing

\section{PCMeVL}

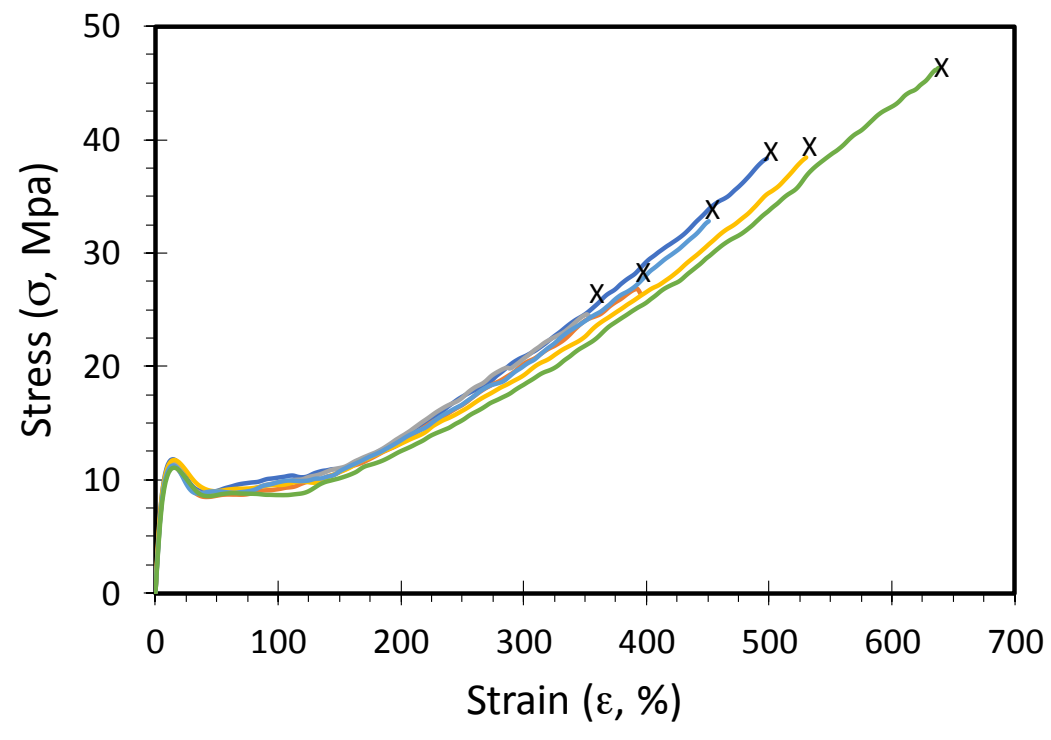

Figure S18. Tensile testing performance of PCMeVL $\left[M_{\mathrm{w}}=130 \mathrm{~kg} \mathrm{~mol}^{-1}\right.$ (MALS)]. Dog-bone shaped samples $(0.2 \mathrm{~mm}$ thickness, ca. $20 \mathrm{~mm}$ gauge length, $5 \mathrm{~mm}$ gauge width, and $15 \mathrm{clamp}$ width) were pulled to the point of break (failure as indicated by X) at a rate of $50.0 \mathrm{~mm} \mathrm{~min}^{-1}$.

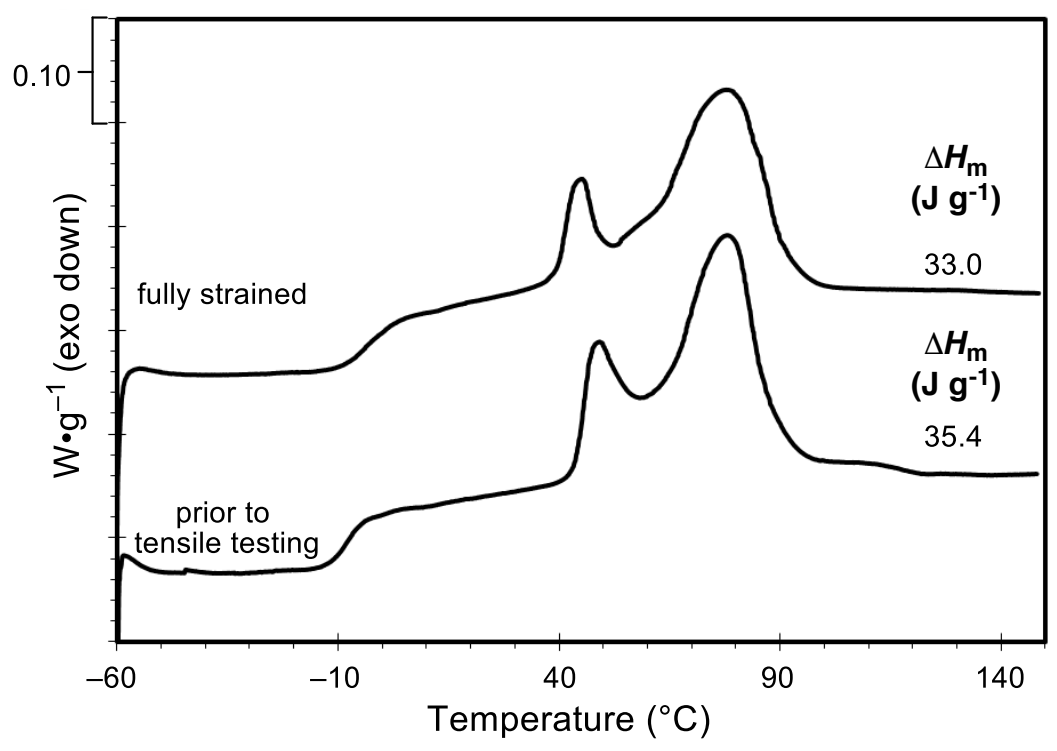

Figure S19. DSC of PCMeVL before and after tensile testing. For one mechanically stretched sample of PCMeVL (with the highest strain at break), a whitening of the sample appeared. We took the DSC of the stretched and broken sample (labeled fully strained) and found little evidence for strain-induced crystallization. 


\section{PCEtVL}

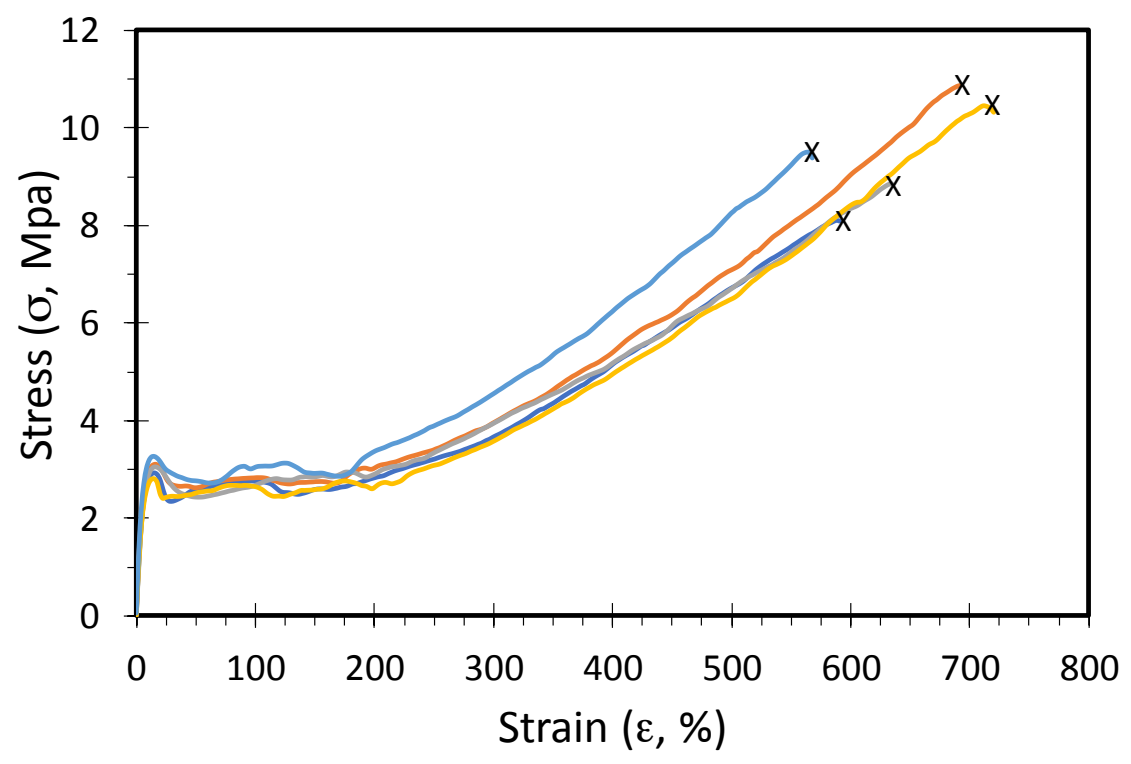

Figure S20. Tensile testing performance of PCEtVL $\left[M_{\mathrm{w}}=86 \mathrm{~kg} \mathrm{~mol}^{-1}\right.$ (MALS)]. Dog-bone shaped samples $(0.2 \mathrm{~mm}$ thickness, ca. $20 \mathrm{~mm}$ gauge length, $5 \mathrm{~mm}$ gauge width, and $15 \mathrm{clamp}$ width) were pulled to the point of break (failure as indicated by X) at a rate of $50.0 \mathrm{~mm} \mathrm{~min}^{-1}$.

\section{PCiPrVL}

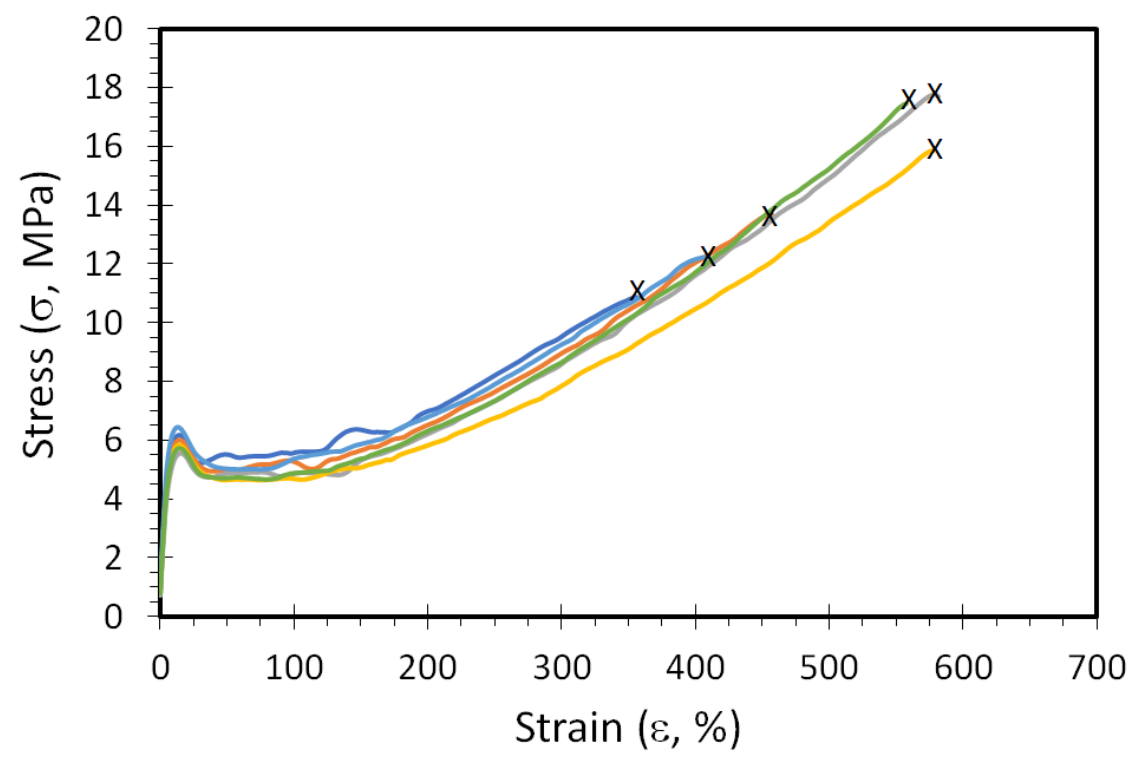

Figure S21. Tensile testing performance of PCiPrVL $\left[M_{\mathrm{w}}=65 \mathrm{~kg} \mathrm{~mol}^{-1}\right.$ (MALS)]. Dog-bone shaped samples $(0.2 \mathrm{~mm}$ thickness, ca. $20 \mathrm{~mm}$ gauge length, $5 \mathrm{~mm}$ gauge width, and $15 \mathrm{clamp}$ width) were pulled to the point of break (failure as indicated by X) at a rate of $50.0 \mathrm{~mm} \mathrm{~min}^{-1}$. 


\section{PCnBuVL}

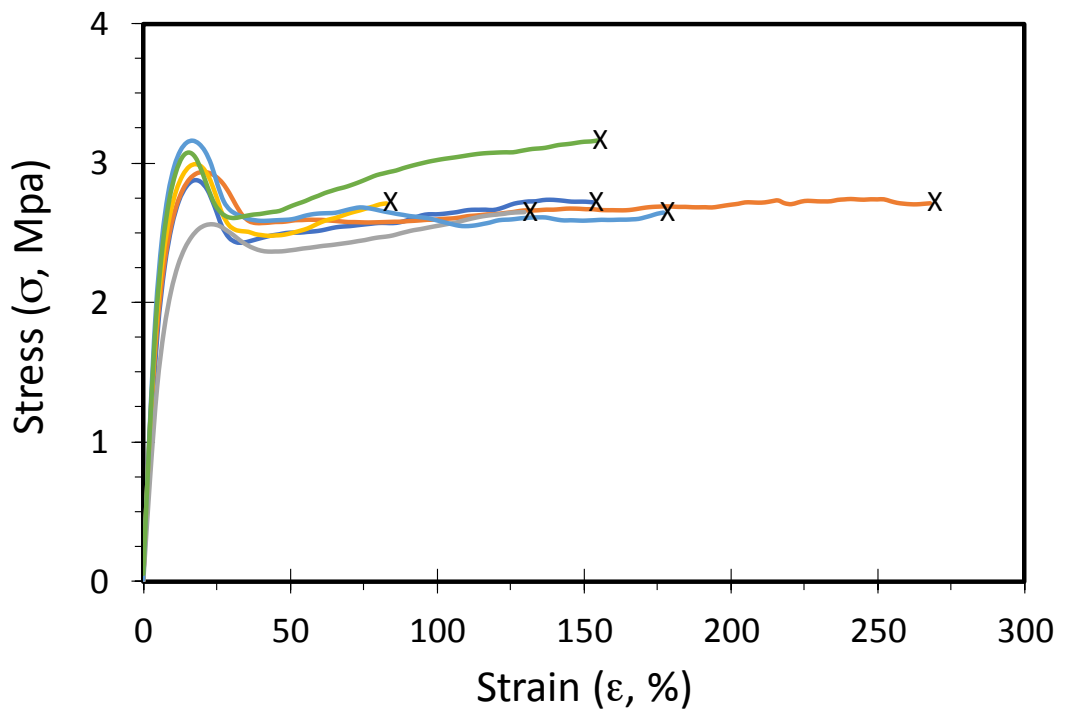

Figure S22. Tensile testing performance of PCnBuVL $\left[M_{\mathrm{w}}=90.3 \mathrm{~kg} \mathrm{~mol}^{-1}\right.$ (MALS) $]$. Dog-bone shaped samples $(0.2 \mathrm{~mm}$ thickness, ca. $20 \mathrm{~mm}$ gauge length, $5 \mathrm{~mm}$ gauge width, and $15 \mathrm{clamp}$ width) were pulled to the point of break (failure as indicated by X) at a rate of $50.0 \mathrm{~mm} \mathrm{~min}^{-1}$. 
a) PCMeVL

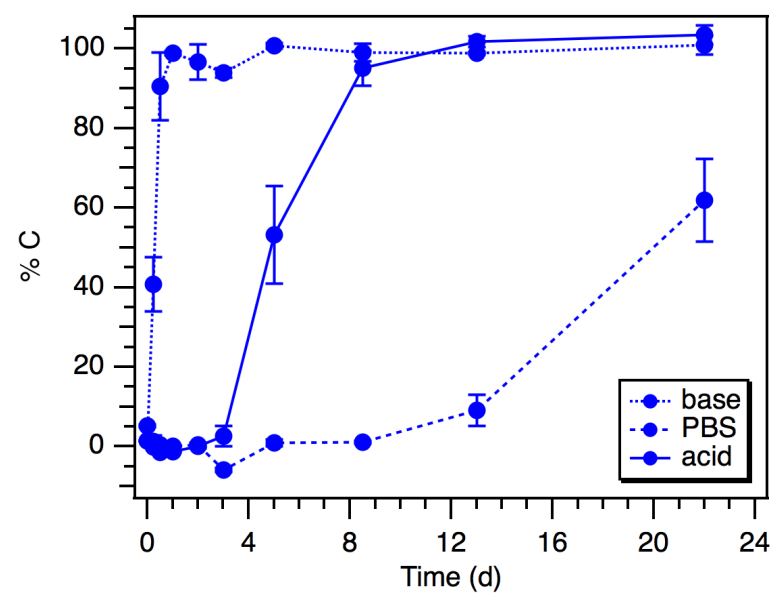

c) PCiPrVL

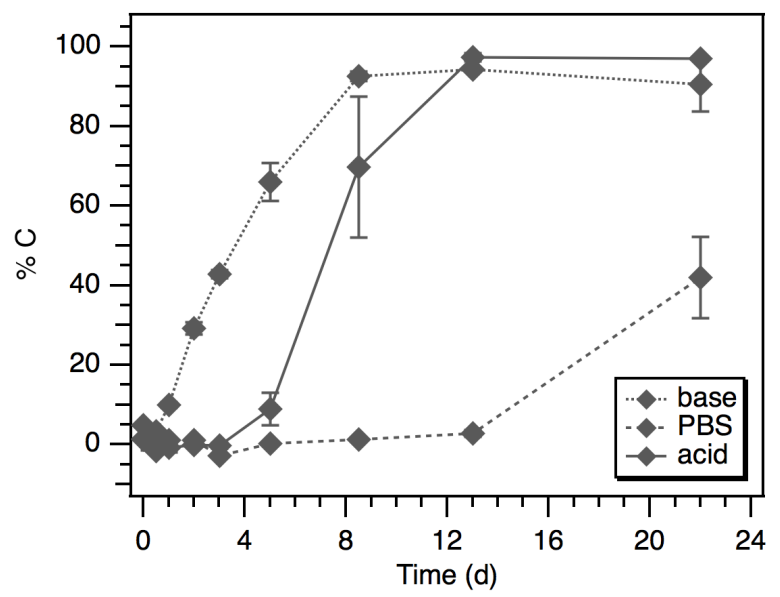

b) PCEtVL

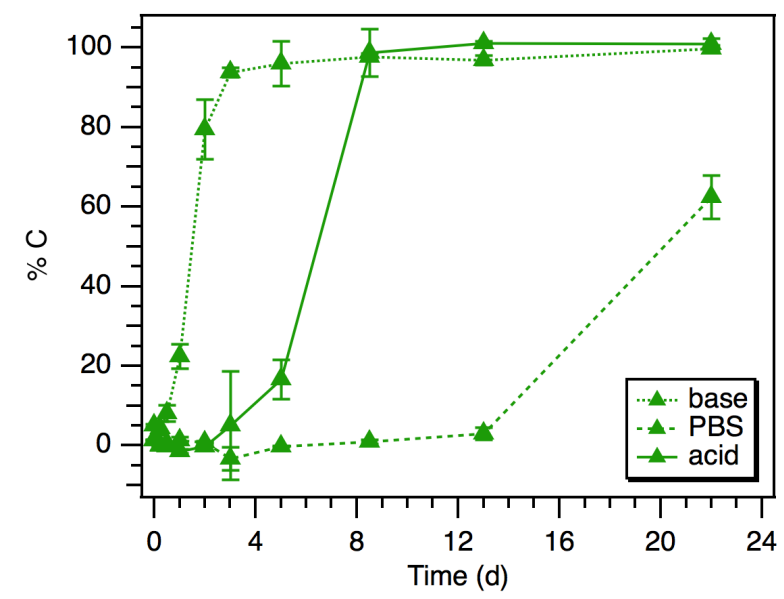

d) PCnBuVL

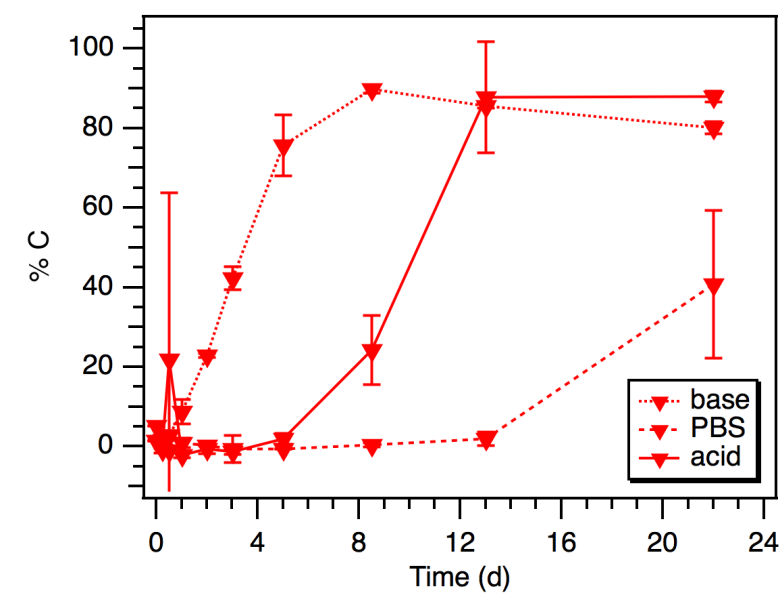

Figure S23. Hydrolytic degradation profiles of high molar mass PCRVLs in $0.1 \mathrm{M} \mathrm{HCl}, 0.1 \mathrm{M}$ $\mathrm{NaOH}$, and 0.1 M phosphate-buffered saline (PBS, $\mathrm{pH} 7.4$ ). The \% $\mathrm{C}$ data were obtained by comparing the total organic carbon content at each timepoint to the amount of carbon present in the initial amount of polymer; each point is an average of triplicate experiments and the error bars represent standard deviations from the mean. 


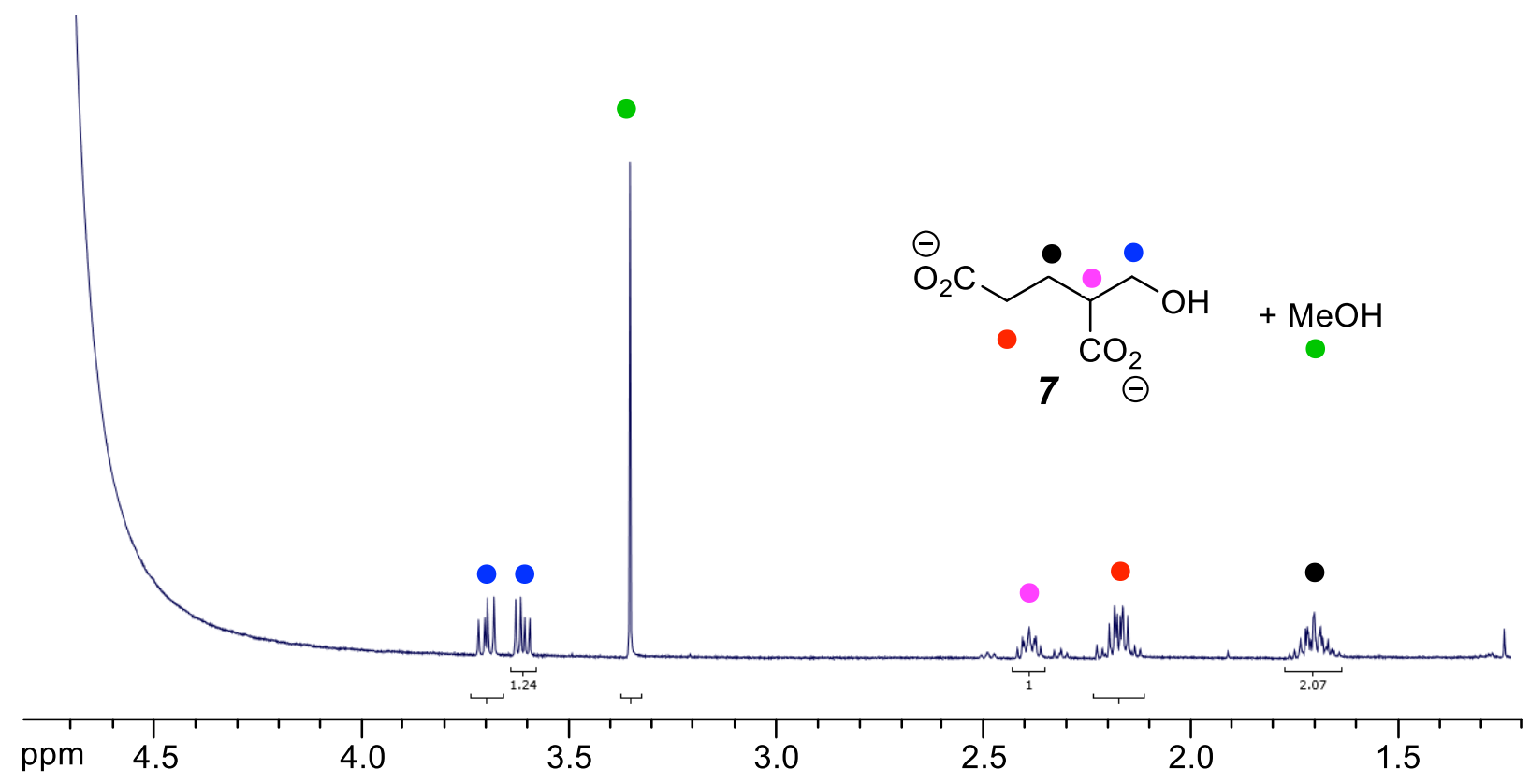

Figure S24. ${ }^{1} \mathrm{H}$ NMR spectrum of the crude product mixture of fully degraded, acidic mixture of PCMeVL at 22 days. The spectrum was referenced to HOD (4.79 ppm), and the presence of $\mathrm{MeOH}$ was confirmed by doping $\mathrm{MeOH}$ into this $\mathrm{D}_{2} \mathrm{O}$ solution. The addition of solid $\mathrm{NaOH}$ (ca. $2 \mathrm{mg}$ ) to this NMR sample produced a spectrum consistent with Figure S25 below.

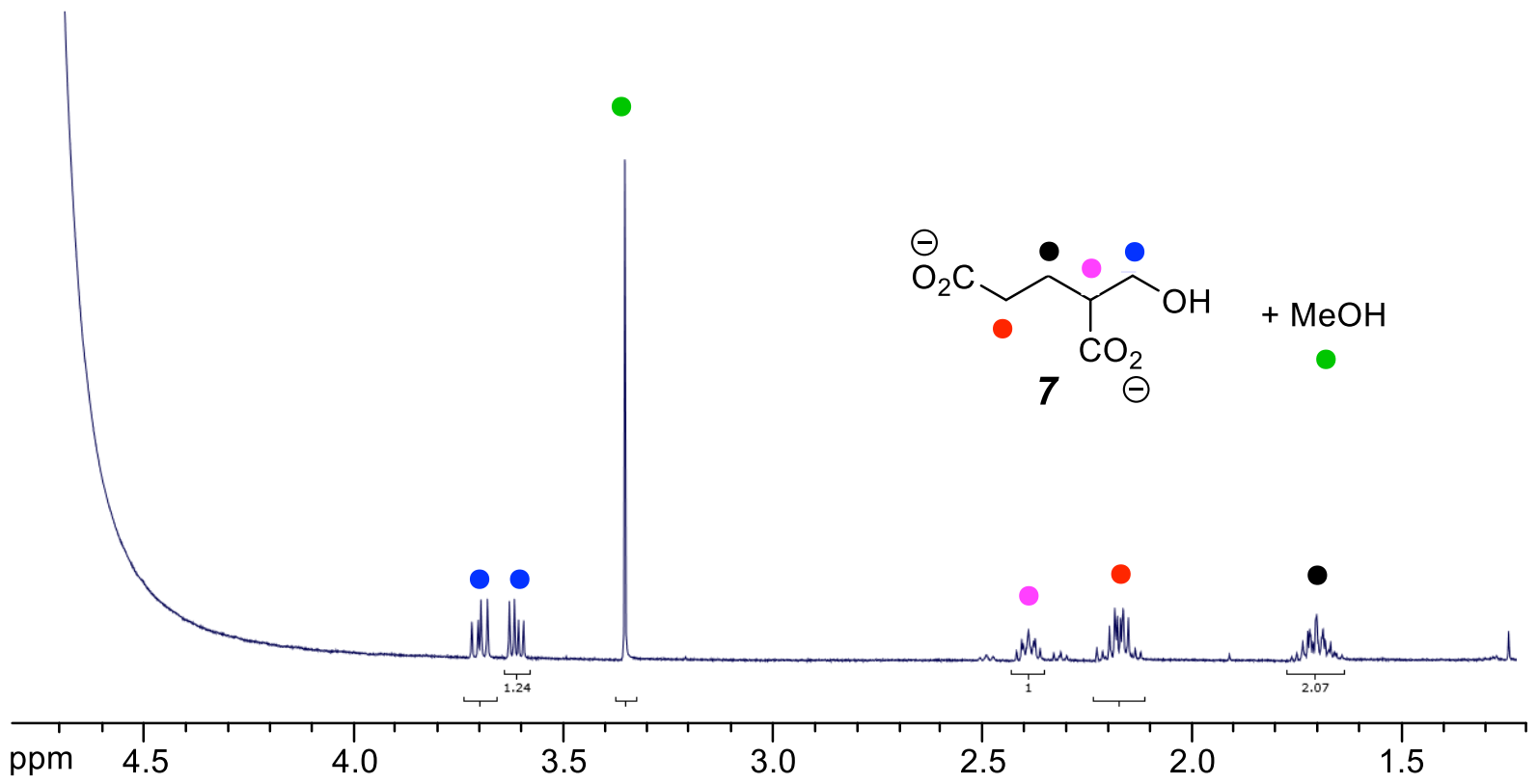

Figure S25. ${ }^{1} \mathrm{H}$ NMR spectrum of the crude product mixture of fully degraded, basic mixture of PCMeVL at 22 days. The spectrum was referenced to HOD (4.79 ppm), and the presence of $\mathrm{MeOH}$ was confirmed by doping $\mathrm{MeOH}$ into this $\mathrm{D}_{2} \mathrm{O}$ solution. 


\section{References}

1 Hoye, T. R.; Hanson, P. R.; Vyvyan, J. R. A practical guide to first-order multiplet analysis in ${ }^{1}$ H NMR spectroscopy. J. Org. Chem. 1994, 59, 4096-4103. (b) Hoye, T. R.; Zhao, H. A method for easily determining coupling constant $(J)$ values: An addendum to "A practical guide to first-order multiplet analysis in ${ }^{1} \mathrm{H}$ NMR spectroscopy." J. Org. Chem. 2002, 67, 4014-4016.

2 Wiley, R. H.; Smith, N. R. Coumalic Acid. Org. Synth. 1951, 31, $23-24$.

3 Fahnhorst, G. W.; Hoye, T. R. A carbomethoxylated polyvalerolactone from malic acid: Synthesis and divergent chemical recycling. ACS Macro Lett. 2018, 143-147.

4 Hendrickson, J. B.; Hussoin, M. S. Reactions of carboxylic acids with "phoshonium anhydrides." J. Org. Chem. 1989, 54, 1144-1149.

5 Campbell, N. R.; Hunt, J. H. 220. Unsaturated lactones. Some esters of aconic and coumalic acids. J. Chem. Soc. 1947, 0, 1176-1179.

6 Yamashita, T.; Nishikawa, H.; Kawamota, T. Scale-up synthesis of a deuterium-labeled ciscyclobutane-1,3-dicarboxylic acid derivative using continuous photo flow chemistry. Tetrahedron 2019, 75, 617-623.

7 Durani, S.; Kapil, R. S. A novel rearrangement reaction: Single-step conversion of 2-( 6carboxy-3-oxoheptyl)-3,4-dihydro-6-methoxynaphthalen-1(2H)-one into 1-(3-carboxybutyl)2- 2-carboxyethyl)-3,4-dihydro-6-methoxynaphthalene. J. Chem. Soc. Perkin. Trans. 1983, 211-217.

8 Messerle, L. Convenient pressure reactors for organometallic reactivity studies. In Experimental Organometallic Chemistry; Wayda, A. L.; Daresnbourg, M. Y.; Eds.; ACS Symposium Series 357; American Chemical Society: Washington DC, pp 198-203, DOI: 10.1021/bk-1987-0357.ch007.

9 Feng, Y.; Coward, J. K. Prodrug forms of N-[(4-Deoxy-4-amino-10methyl)pteroyl]glutamate- $\gamma-[\psi \mathrm{P}(\mathrm{O})(\mathrm{OH})]$-glutarate, a potent inhibitor of Folylpoly- $\gamma$ glutamate synthetase: Synthesis and hydrolytic stability. J. Med. Chem., 2006, 49, 770-788.

${ }^{10}$ Fahnhorst, G. W.; Stasiw, D. E.; Tolman, W. B.; Hoye, T. R. Isomerization of linear to hyperbranched polymers: Two isomeric lactones converge via metastable isostructural polyesters to a highly branched analogue. ACS Macro Lett. 2018, 7, 1144-1148.

\section{Copies of ${ }^{1} \mathrm{H}$ and ${ }^{13} \mathrm{C}$ NMR Spectra}




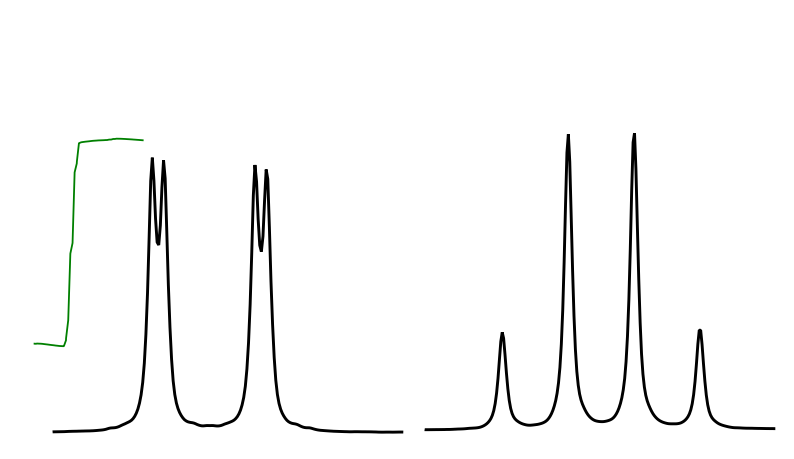

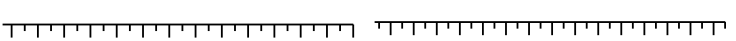

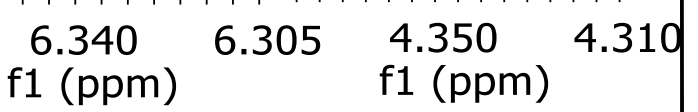

6.340
$\mathrm{f} 1(\mathrm{ppm})$ 7.800
$\mathrm{f} 1(\mathrm{ppm})$

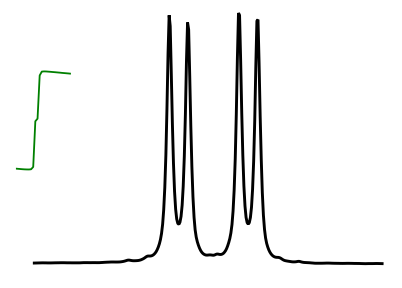

7.750 8.304
f1 (ppm)

8.276 (1)

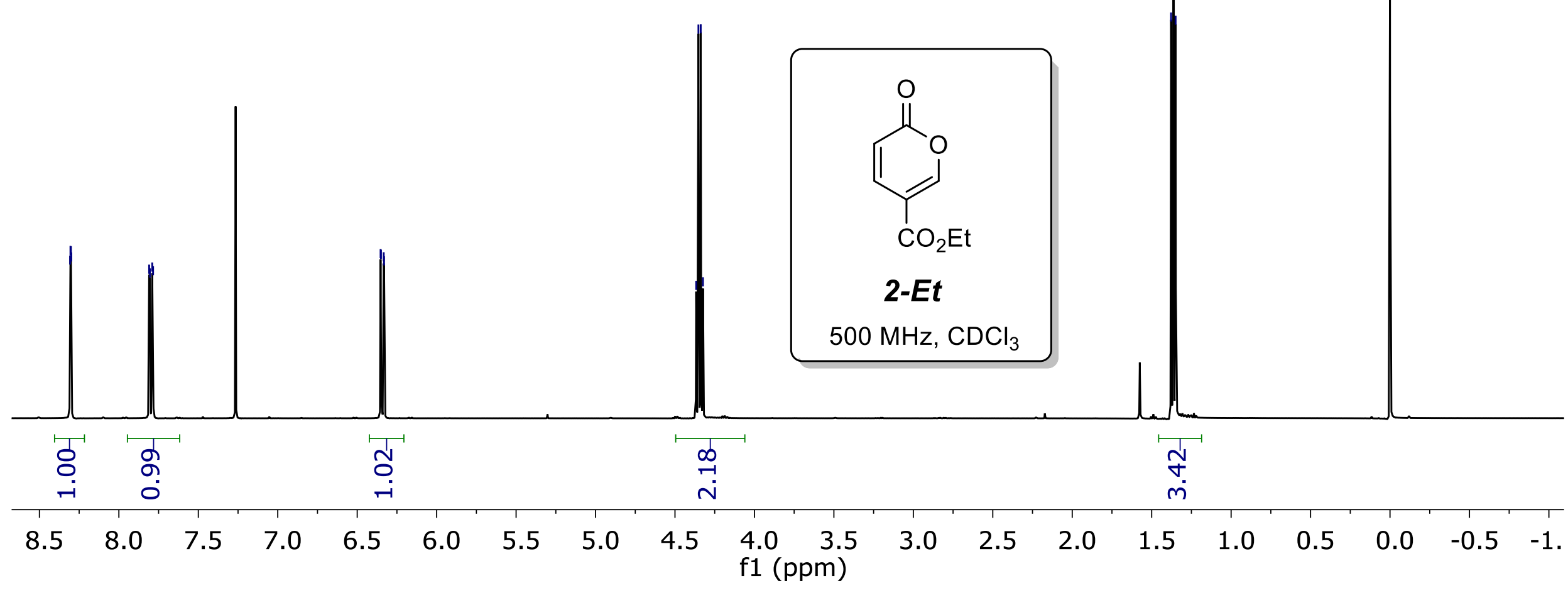


$\begin{array}{ccc}-1 & 0 & N \\ m & 0 & 0 \\ 0 & 0 & 0 \\ -1 & 0 \\ \mid & 1\end{array}$

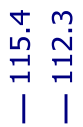

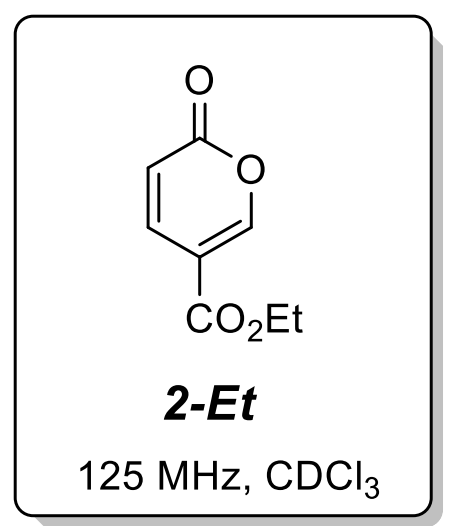



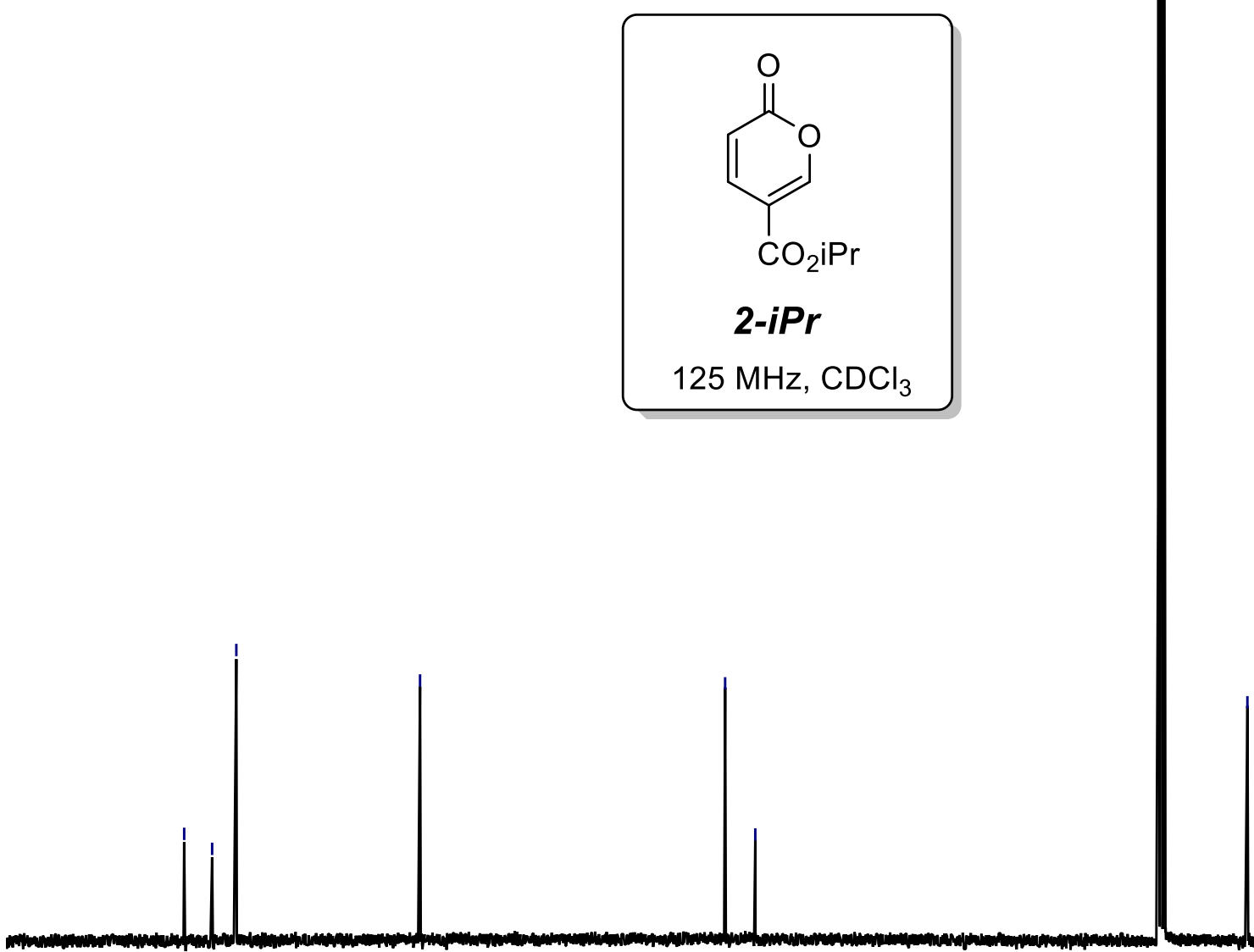

$\begin{array}{lllllllll}170 & 160 & 150 & 140 & 130 & 120 & 110 & 100 & 90 \\ \mathrm{f} 1(\mathrm{ppm})\end{array}$

$70 \quad 60 \quad 50 \quad 40$

3030

3020

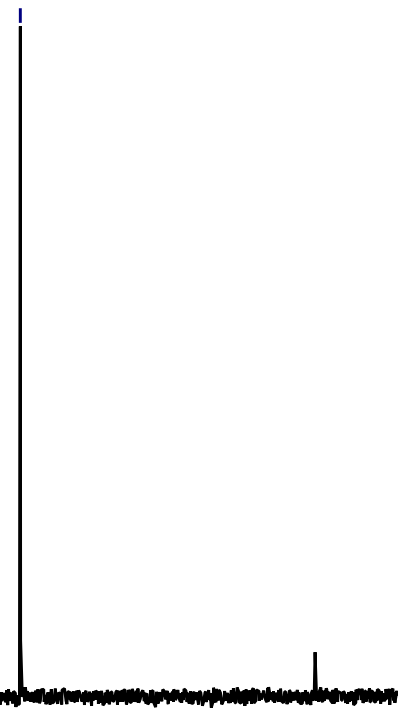




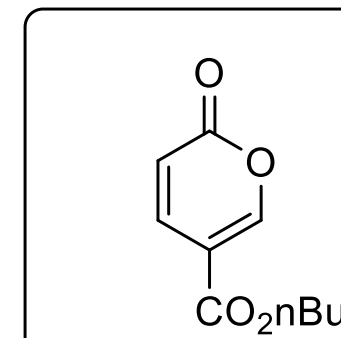

2-nBu $125 \mathrm{MHz}, \mathrm{CDCl}_{3}$

$\begin{array}{lllllllll}170 & 160 & 150 & 140 & 130 & 120 & 110 & 100 & 90 \\ 81(\mathrm{ppm})\end{array}$



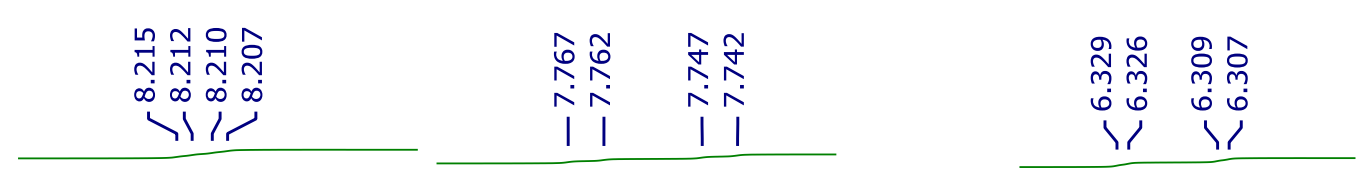
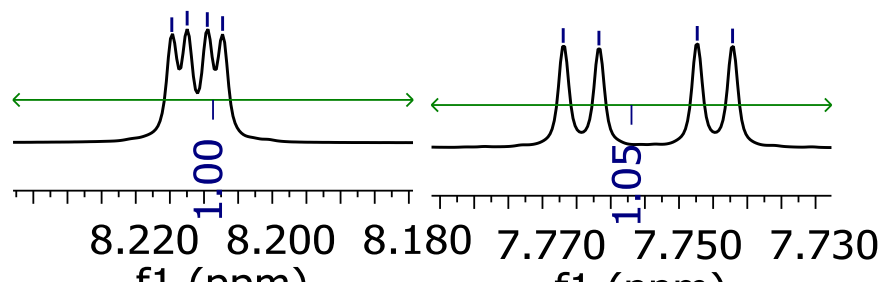
f1 (ppm) f1 (ppm)

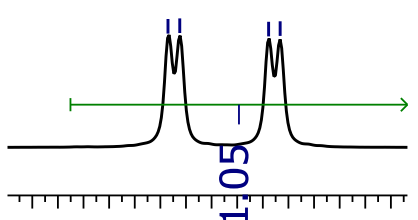

6.3356 .3106 .285 f1 (ppm)
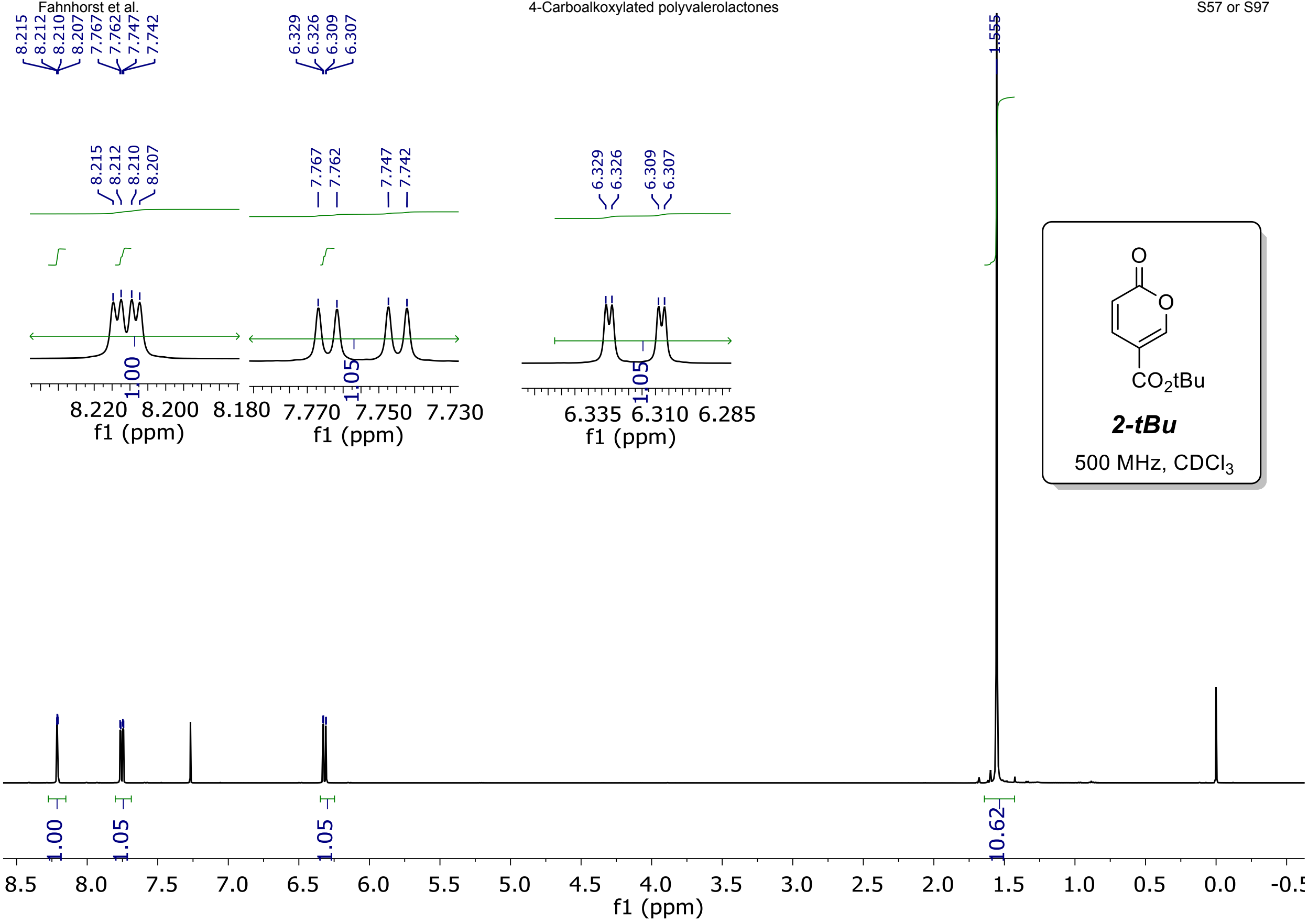

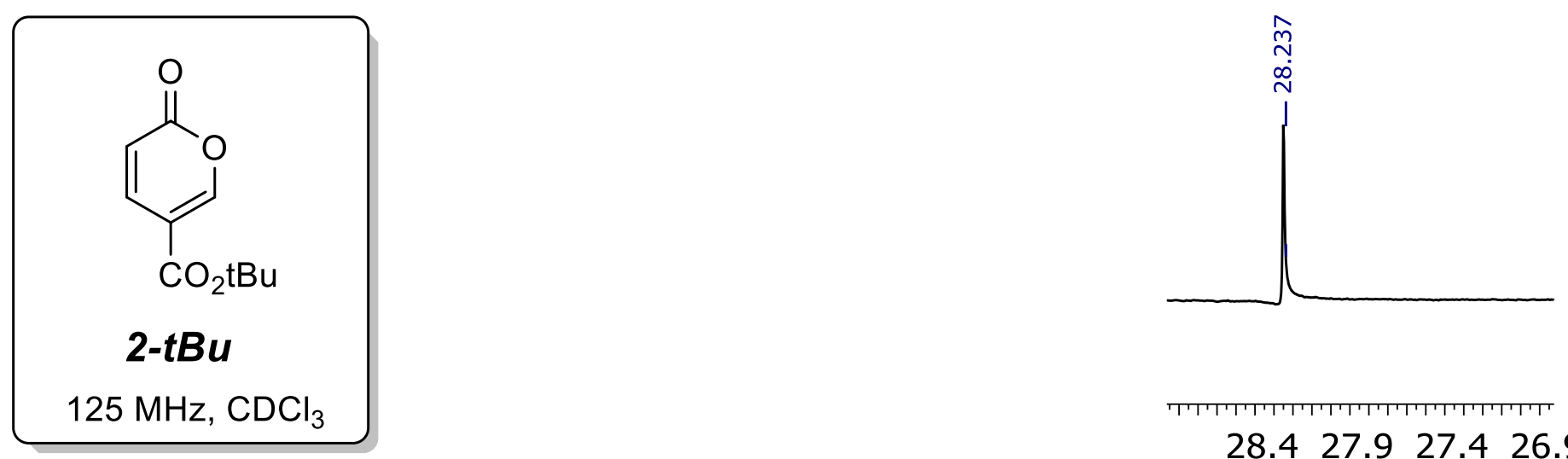

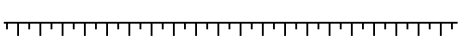

$28.4 \quad 27.927 .4 \quad 26.9$ f1 (ppm)

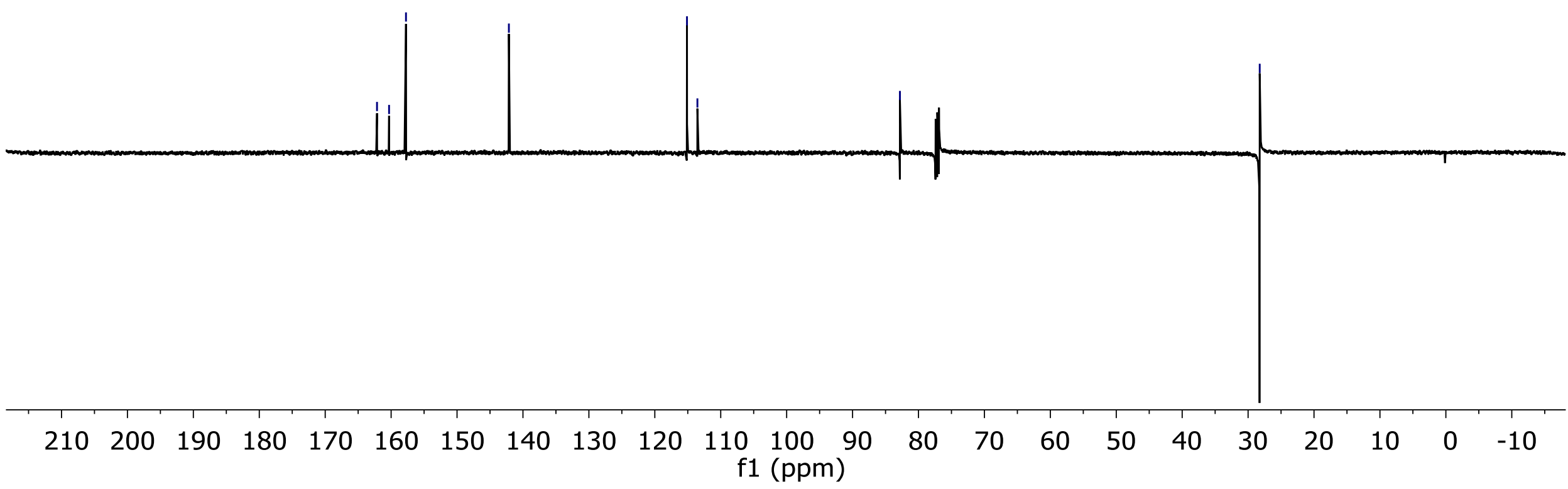




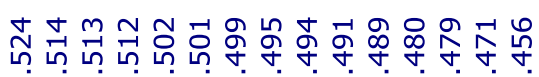

$\sqrt{\dot{2} 0}$

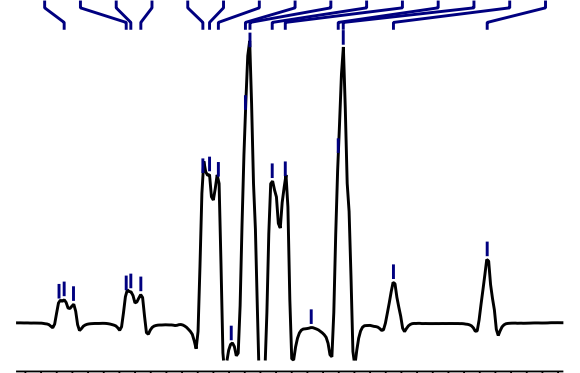

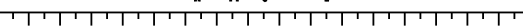

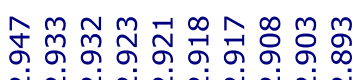
nับ

12

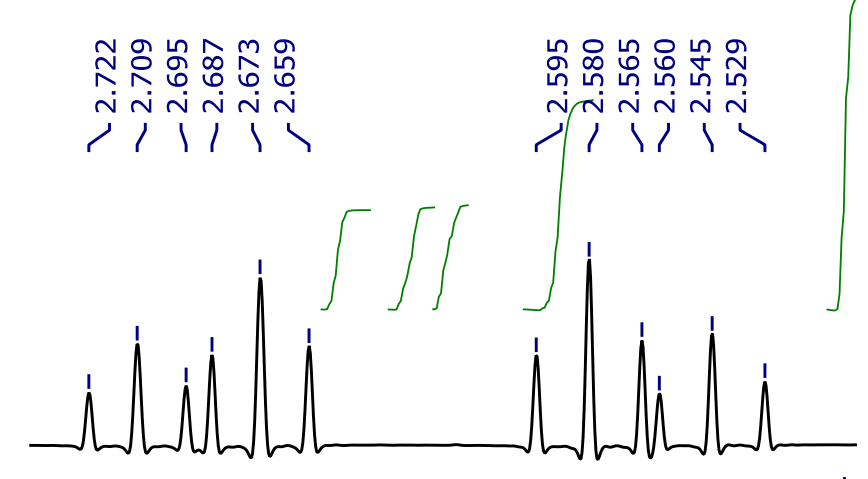

$\begin{array}{llll}4.520 & 4.495 & 4.470 & 4.445\end{array}$ f1 (ppm)

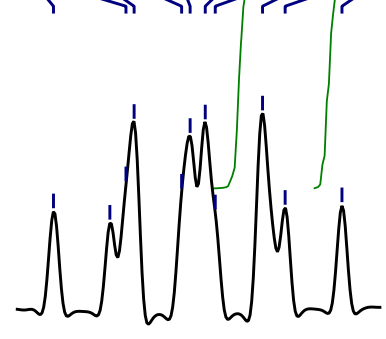

2.9402 .9152 .890 f1 (ppm)
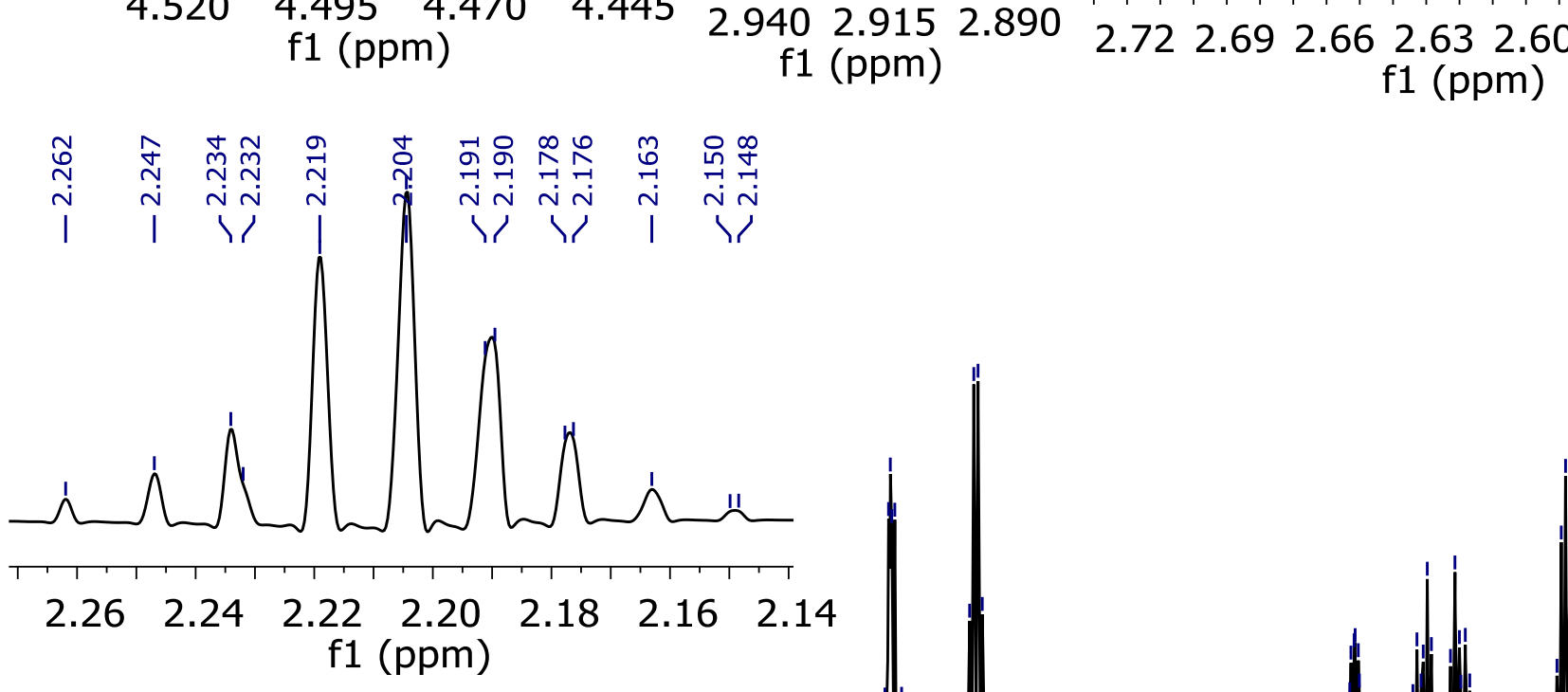

f1 (ppm)

$\begin{array}{lll}2.57 & 2.54 & 2.51\end{array}$

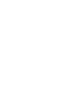



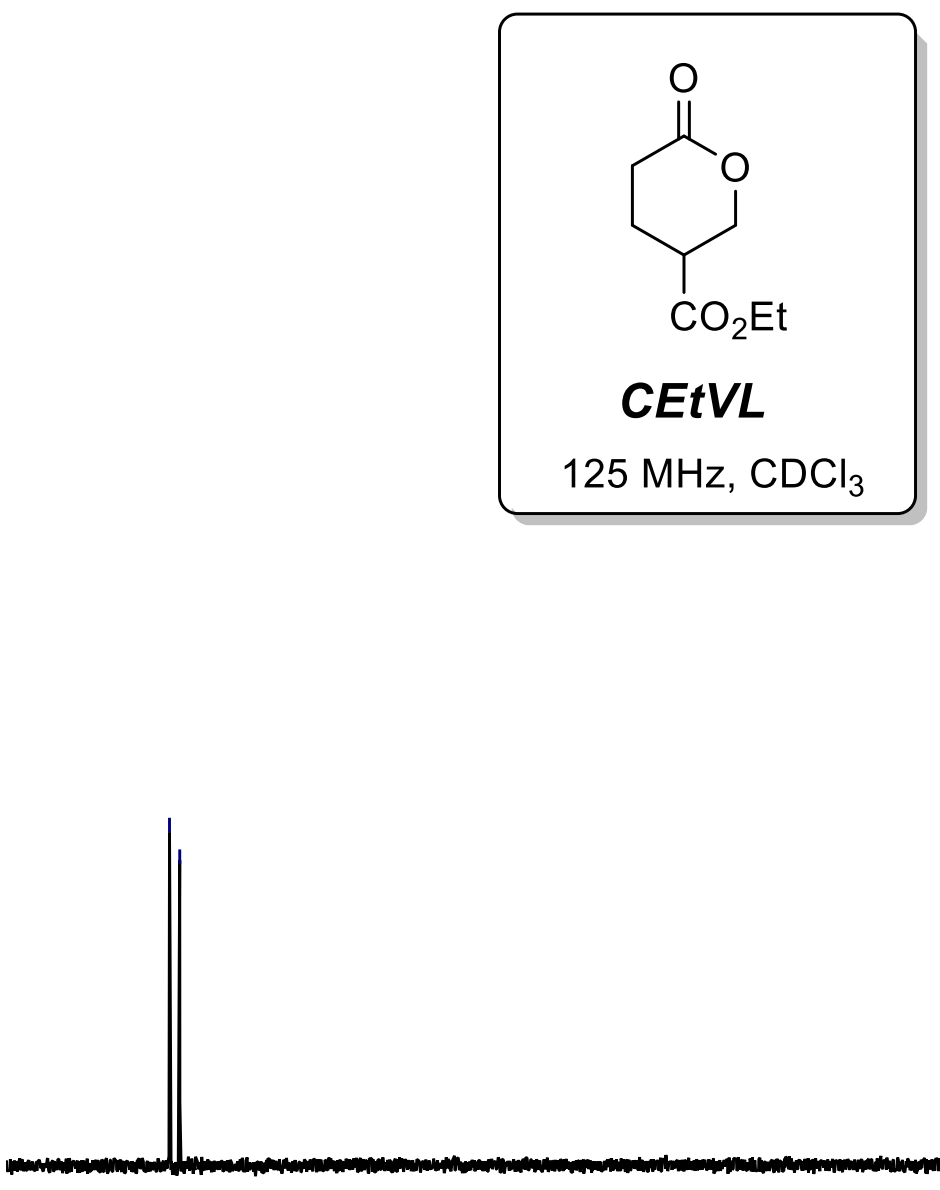

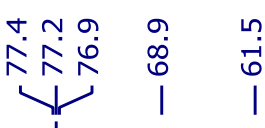

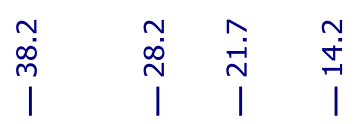

\section{$125 \mathrm{MHz}, \mathrm{CDCl}_{3}$}

$\begin{array}{llll}170 & 160 & 150 & 140\end{array}$

$\begin{array}{llllll}130 & 120 & 110 & 100 & \begin{array}{c}90 \\ \mathrm{f} 1(\mathrm{ppm})\end{array} & 80\end{array}$

$\begin{array}{llllllll}60 & 50 & 40 & 30 & 20 & 10 & 0 & -1\end{array}$



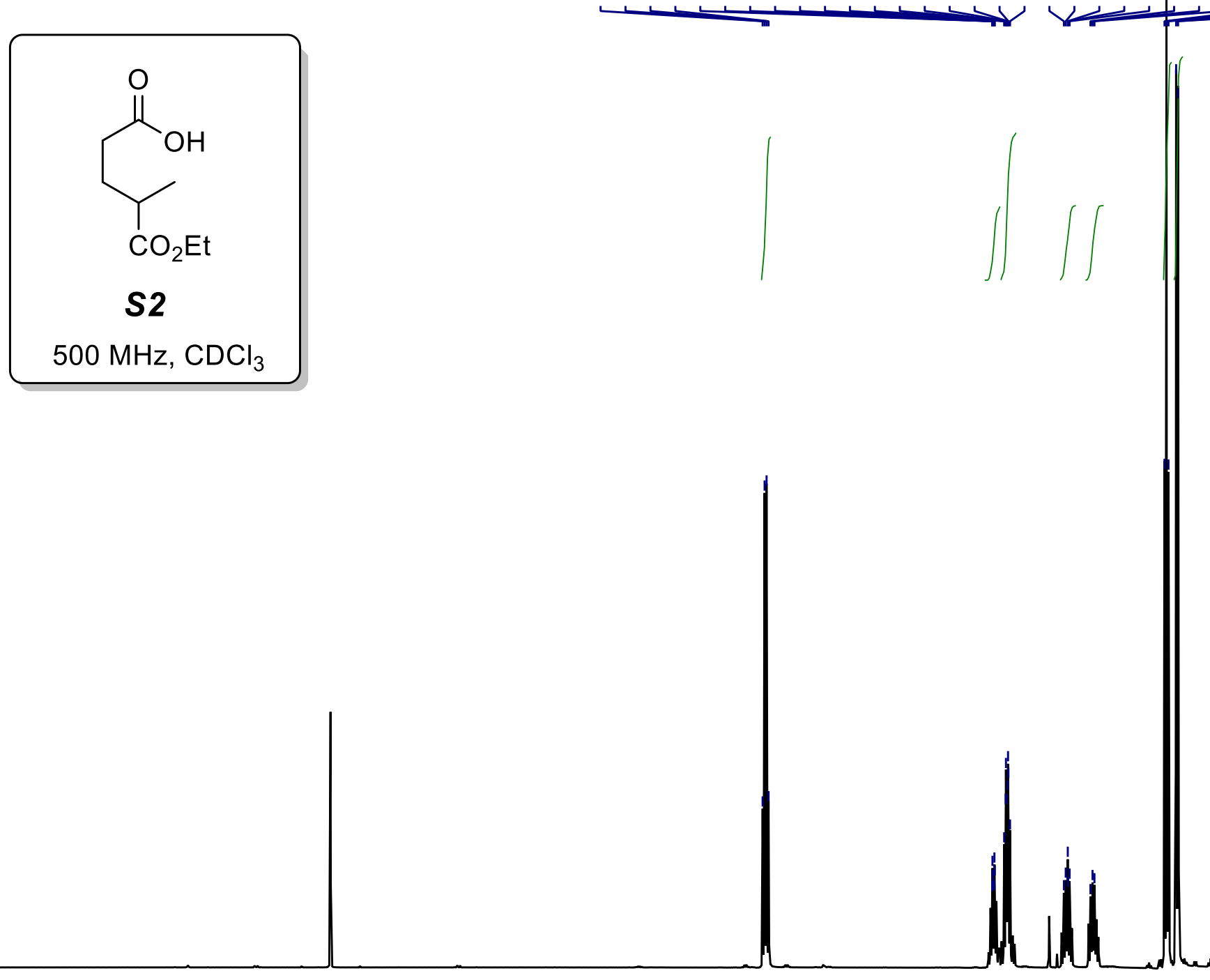

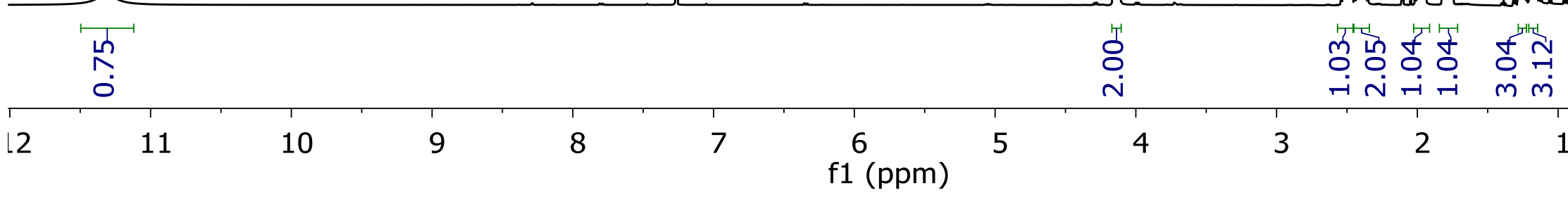

1

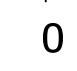




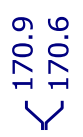

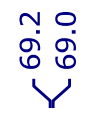

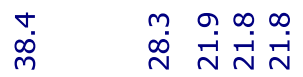

$1 \quad 1$
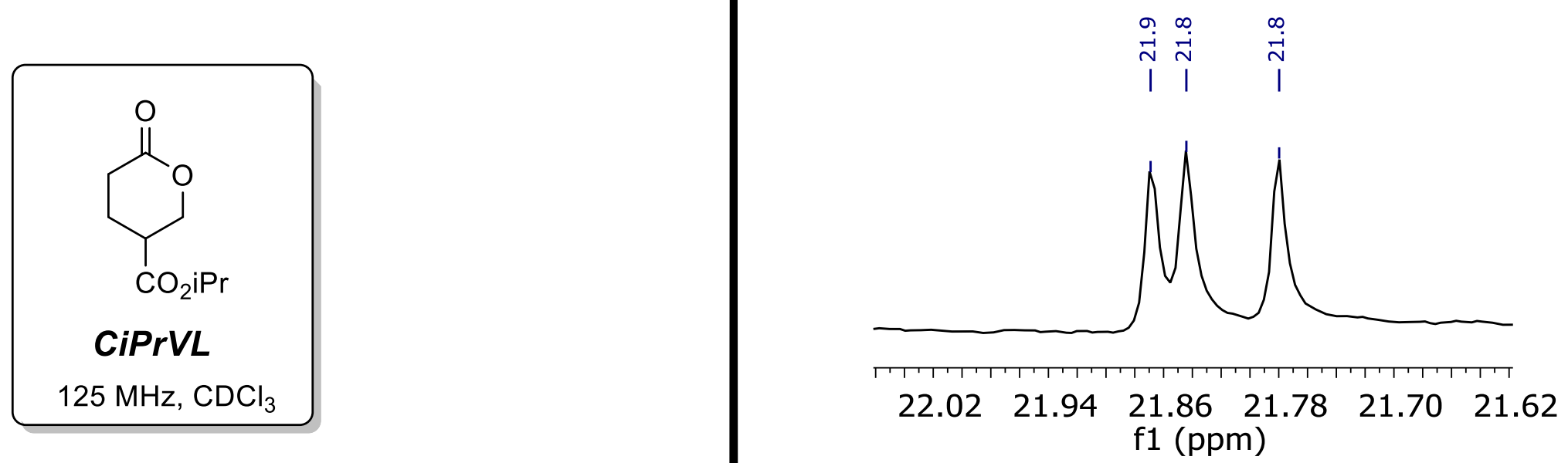

\begin{tabular}{|c|c|c|c|c|c|c|c|c|c|c|c|c|c|c|c|c|c|c|}
\hline 180 & 170 & 160 & 150 & 140 & 130 & 120 & 110 & 100 & $\begin{array}{c}90 \\
\mathrm{f} 1(\mathrm{ppm})\end{array}$ & )$^{80}$ & 70 & 60 & 50 & 40 & 30 & 20 & 10 & 0 \\
\hline
\end{tabular}



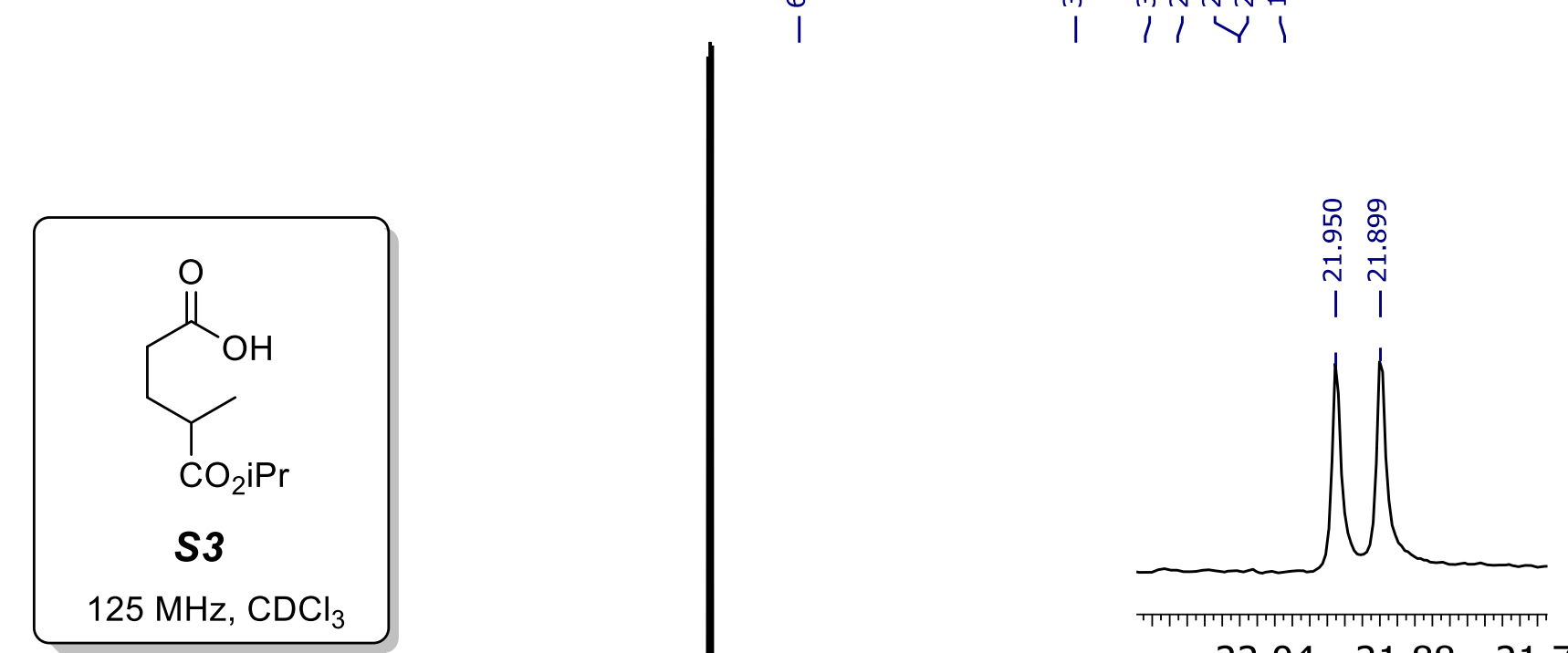

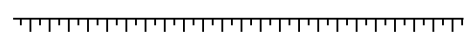

$22.04 \quad 21.88 \quad 21.72$ f1 (ppm)

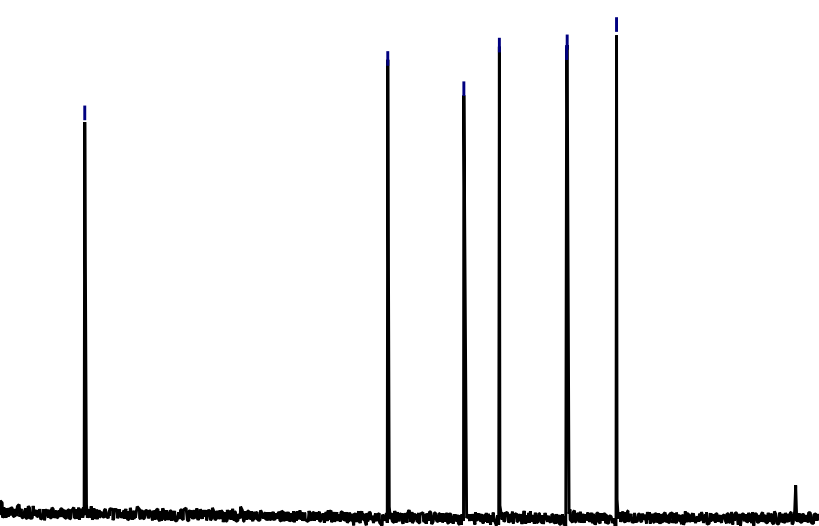

$\begin{array}{lllllllllllllllllllllll}210 & 200 & 190 & 180 & 170 & 160 & 150 & 140 & 130 & 120 & \begin{array}{c}110 \\ \mathrm{f} 1\end{array}(\mathrm{ppm})\end{array}$




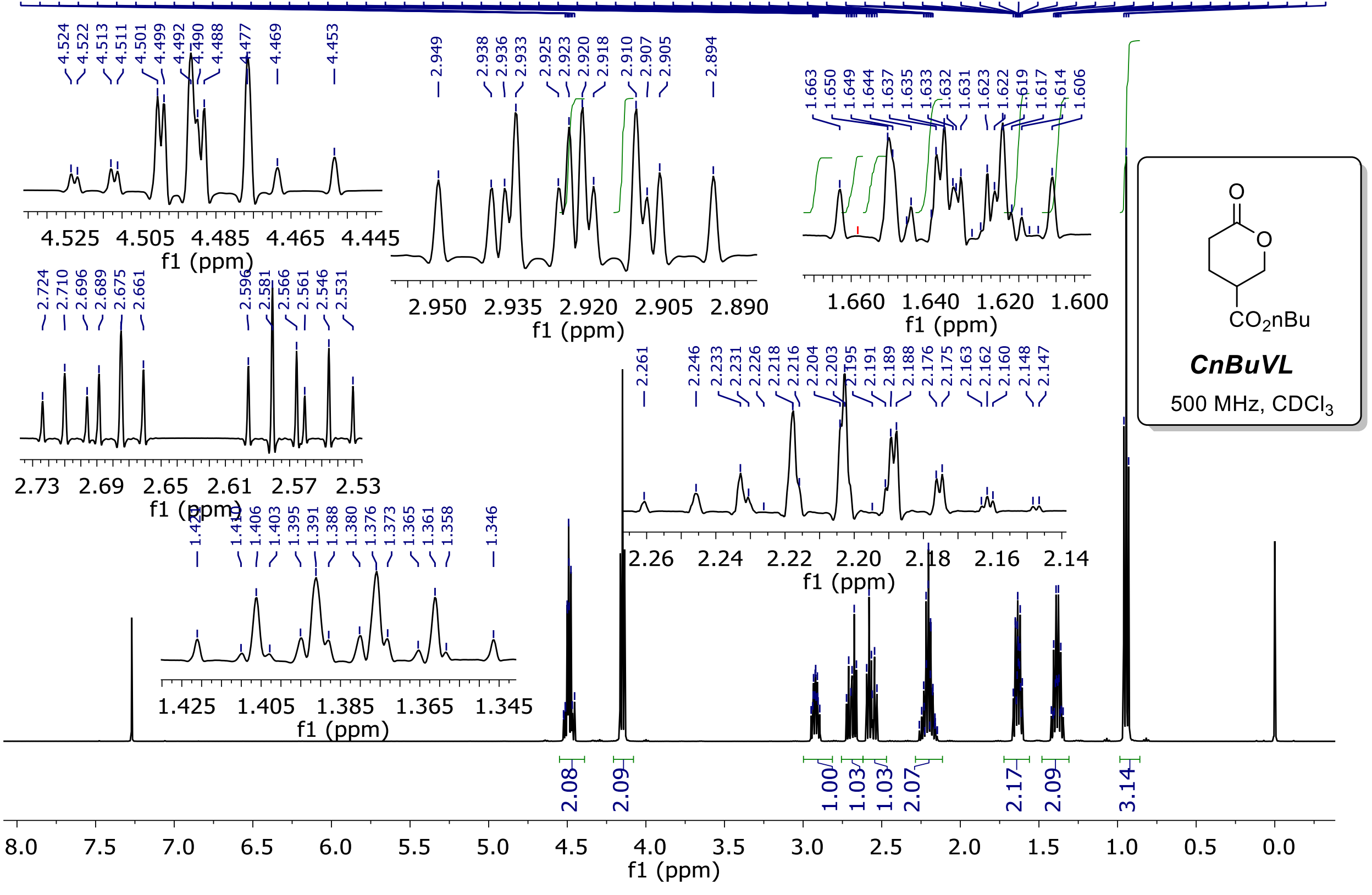




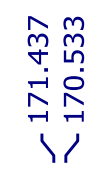

ֻั.

ஸे กี่

$\begin{array}{lll}\infty & 0 \\ 0 & 1 & 1\end{array}$

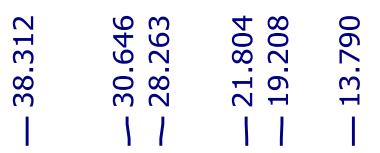
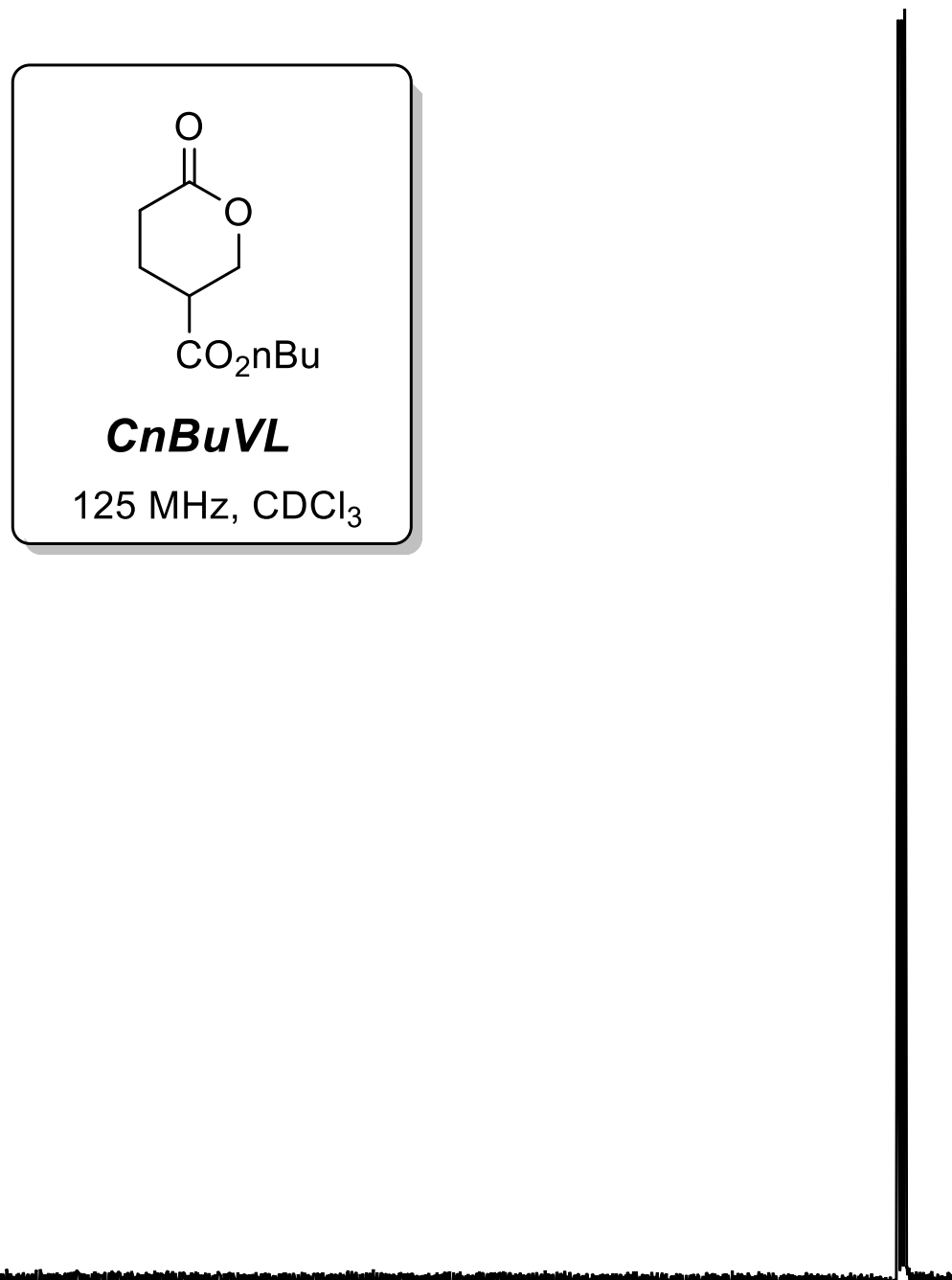

(1)

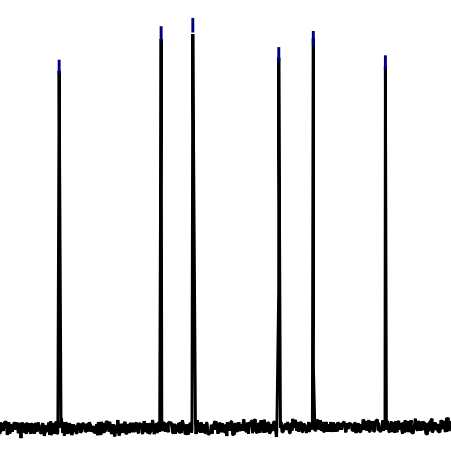

$\begin{array}{llllllll}70 & 60 & 50 & 40 & 30 & 20 & 10 & 0\end{array}$



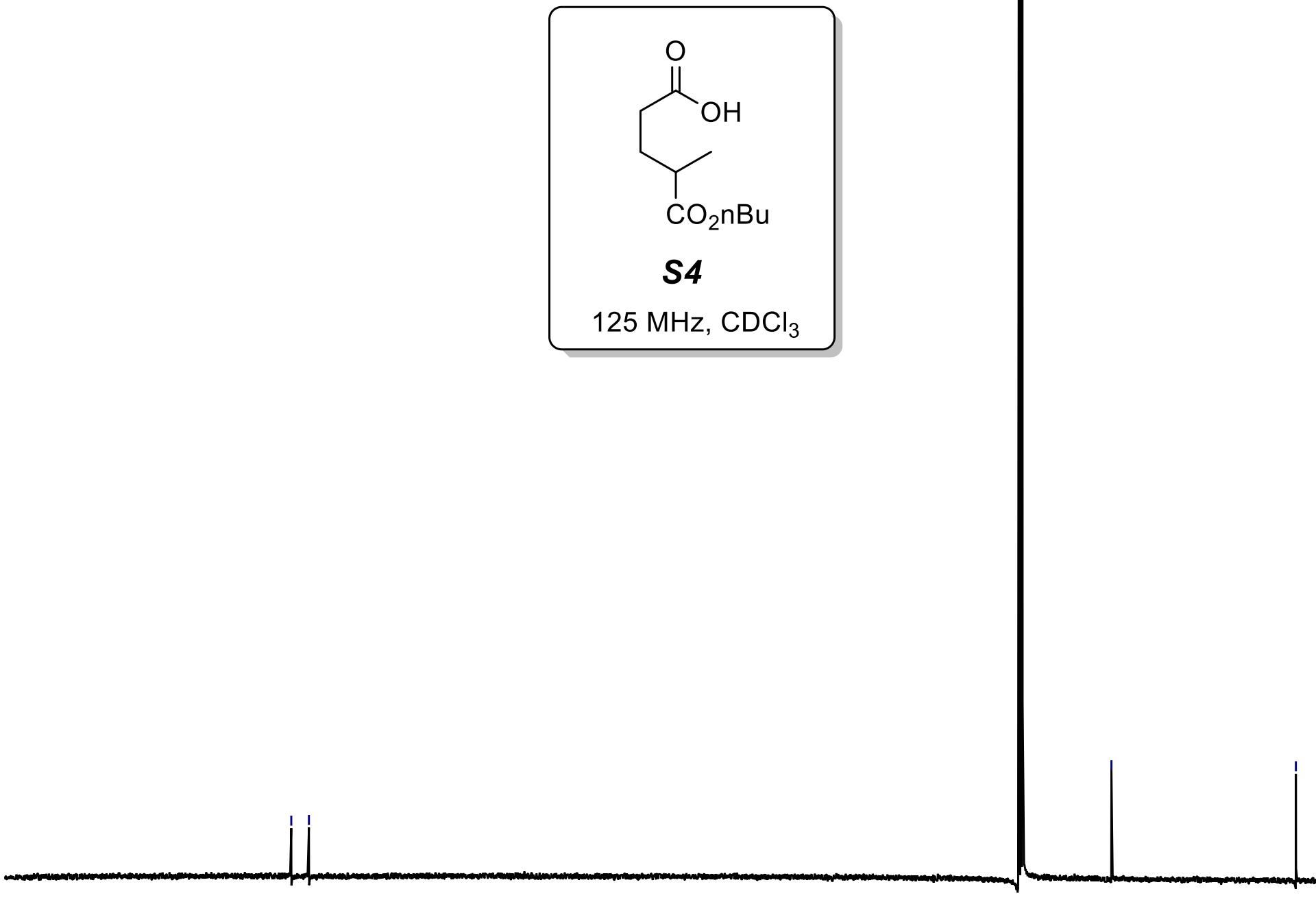

$\begin{array}{lllllllllllllllllllllll}210 & 200 & 190 & 180 & 170 & 160 & 150 & 140 & 130 & 120 & \begin{array}{c}110 \\ \mathrm{f} 1\end{array}(\mathrm{ppm})\end{array}$




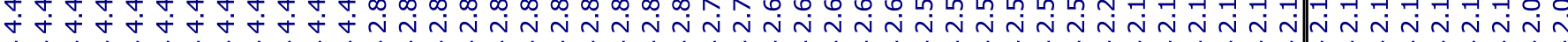
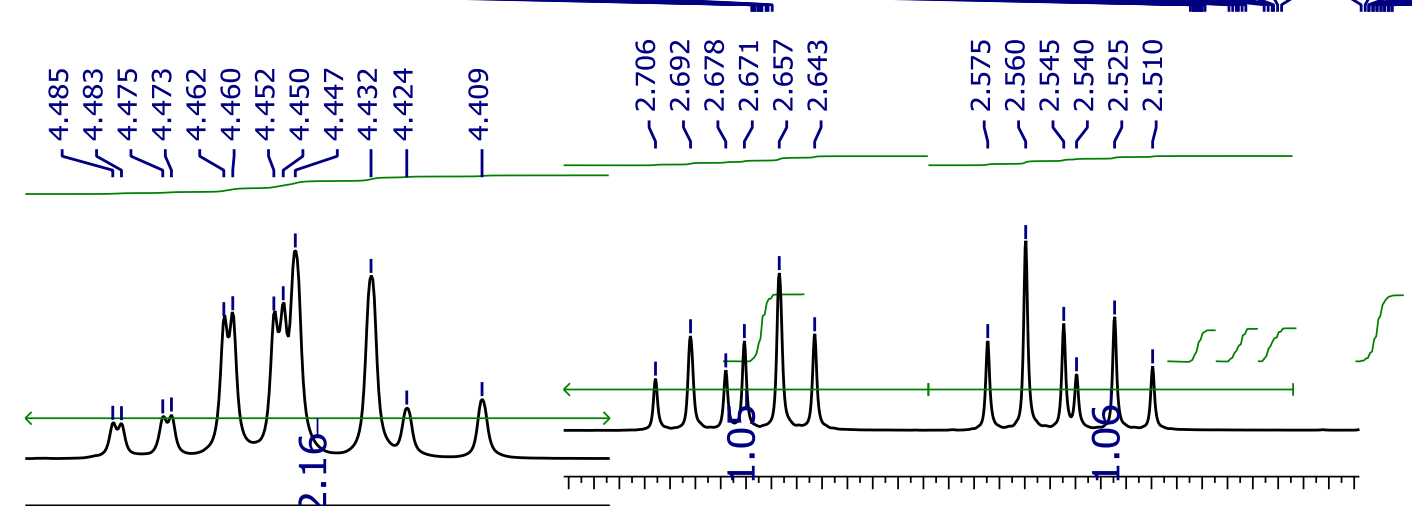

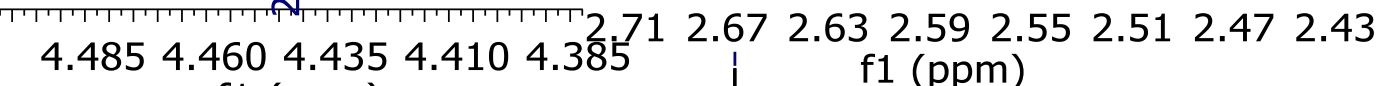
f1 (ppm)

员

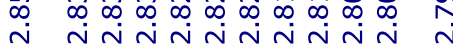

i nny

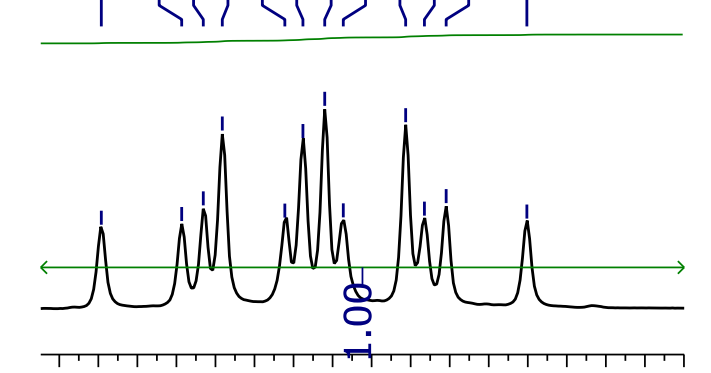

$\begin{array}{lllll}2.855 & 2.835 & 2.815 & 2.795 & 2.775\end{array}$ f1 (ppm)
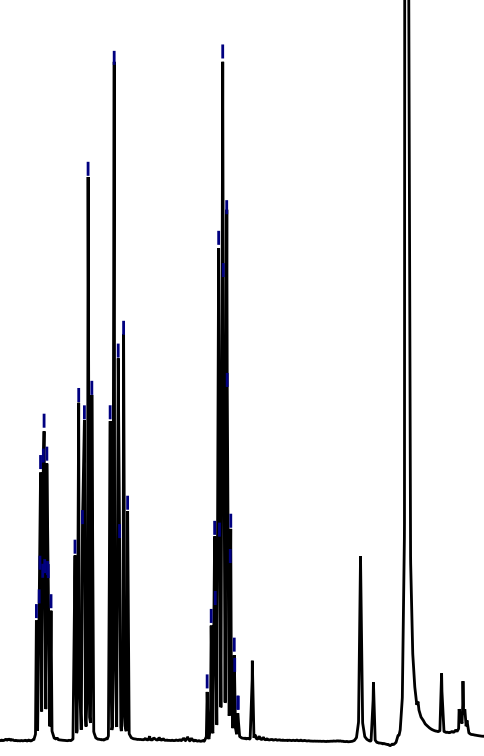

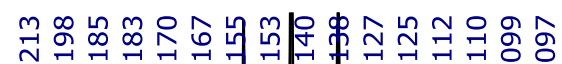

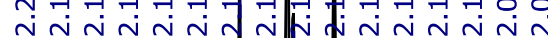

N n $n$ N n n n n

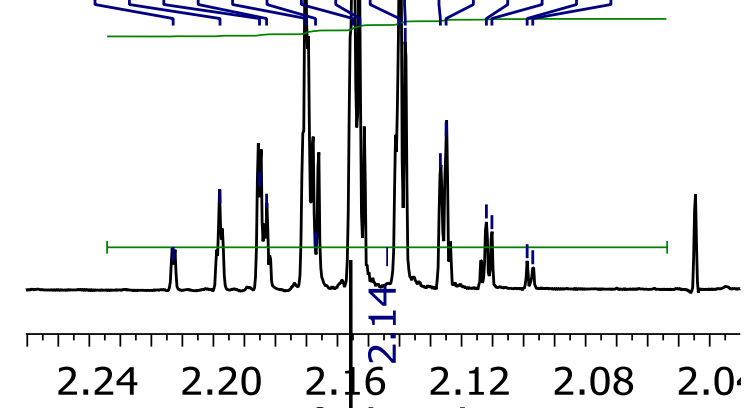

2.24

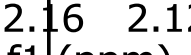

2.08

2.0 f1 (ppm)

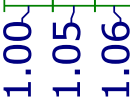

$2.5 \quad 2.0$

4.5

4.0

$3.5 \quad 3.0$

2.5
के<smiles>CCCOC(=O)C1CCC(=O)OC1</smiles>

CtBuVL

$500 \mathrm{MHz}, \mathrm{CDCl}_{3}$

\begin{tabular}{|c|c|c|c|c|c|c|c|c|c|c|c|c|c|c|c|c|c|}
\hline 7.5 & 7.0 & 6.5 & 6.0 & 5.5 & 5.0 & 4.5 & 4.0 & $\begin{array}{c}3.5 \\
\text { f1 (pp }\end{array}$ & $n^{3.0}$ & 2.5 & 2.0 & 1.5 & 1.0 & 0.5 & 0.0 & -0.5 & -1.0 \\
\hline
\end{tabular}




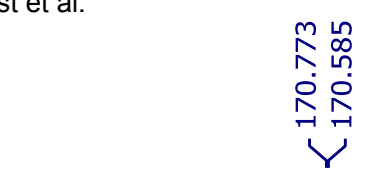<smiles>CC(C)(C)OC(=O)C1CCC(=O)OC1</smiles>

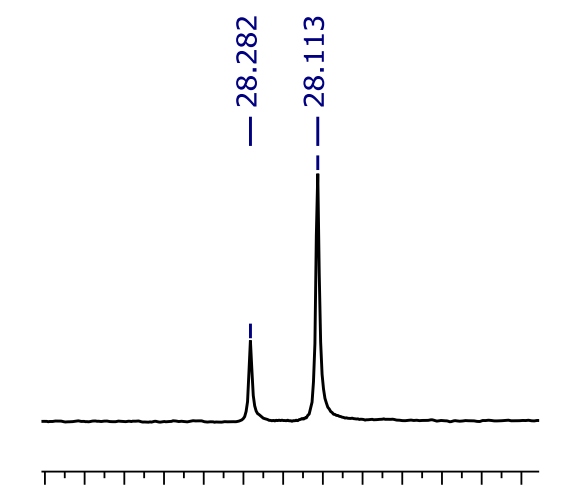

$\begin{array}{lllll}28.8 & 28.5 & 28.2 & 27.9 & 27.6\end{array}$

$$
\text { f1 (ppm) }
$$

$125 \mathrm{MHz} \mathrm{CDCl}_{3}$

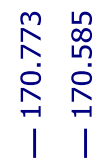

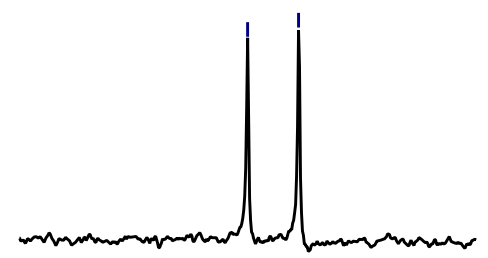

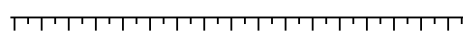

$\begin{array}{lll}171.2 & 170.6 \quad 170.0\end{array}$ f1 (ppm) 

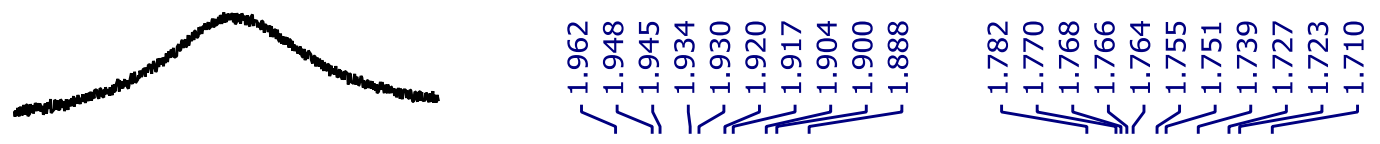

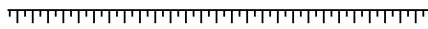

\section{$\begin{array}{lll}12.0 & 11.3 & 10.6 \quad 9.9\end{array}$ f1 (ppm)}

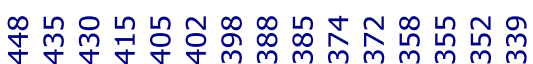

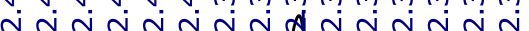
is

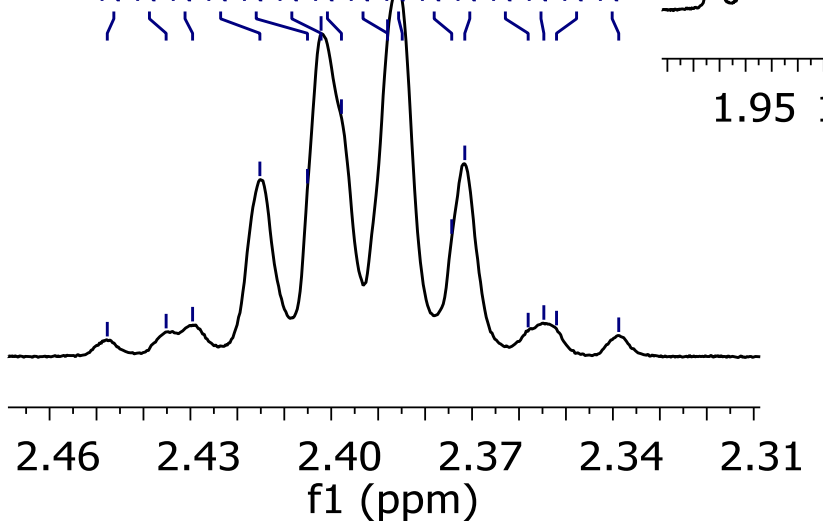

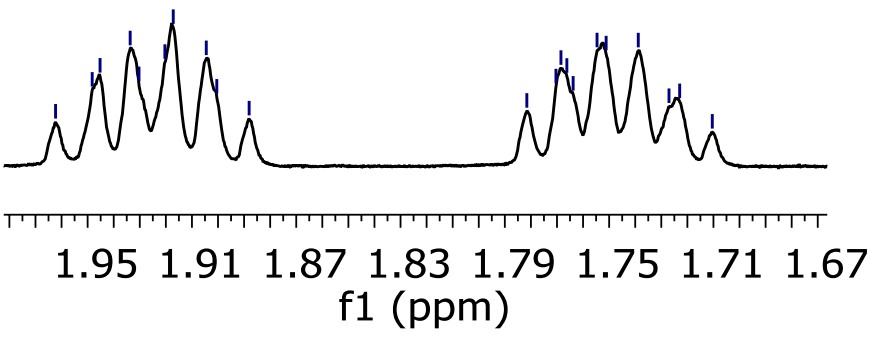

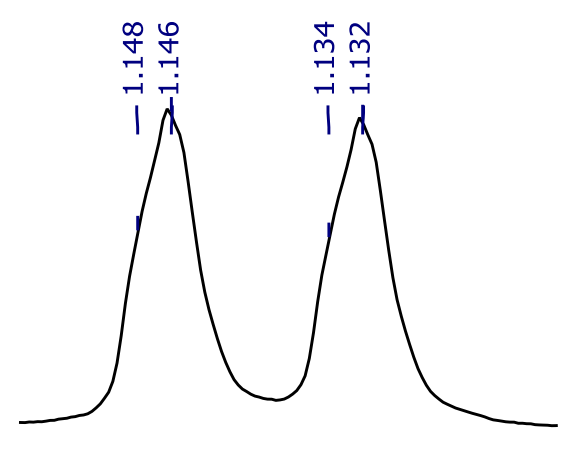

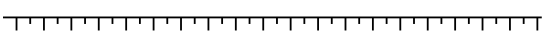

$\begin{array}{llll}1.148 & 1.138 & 1.128 & 1.118\end{array}$ f1 (ppm)

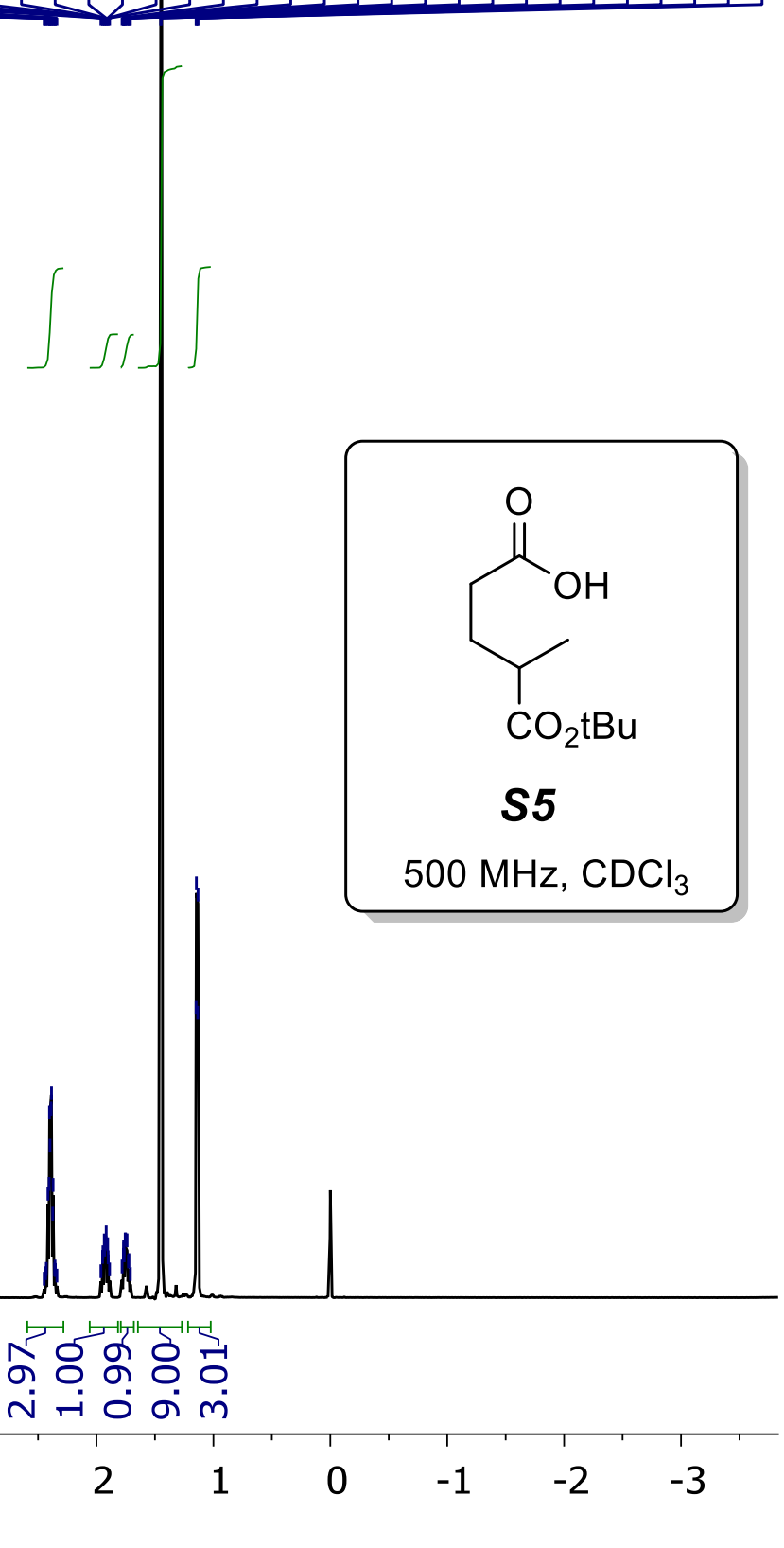



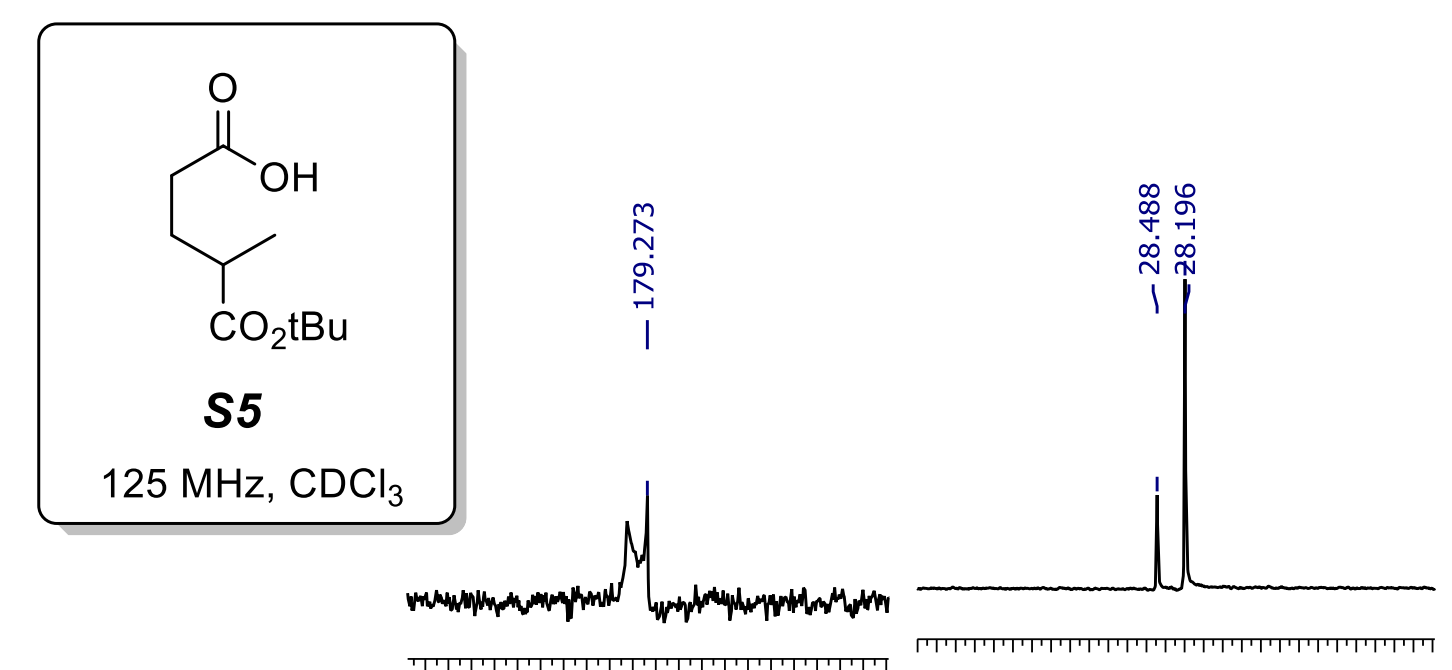

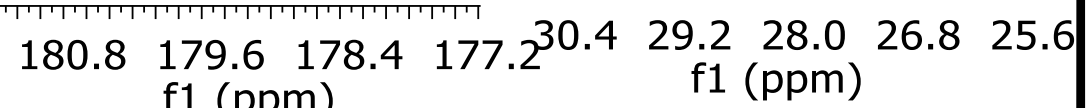
f1 (ppm)

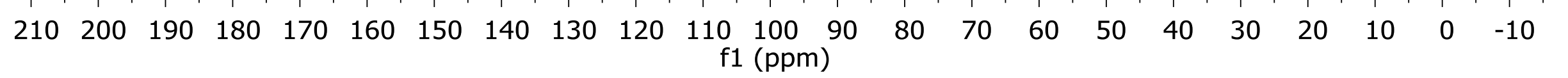




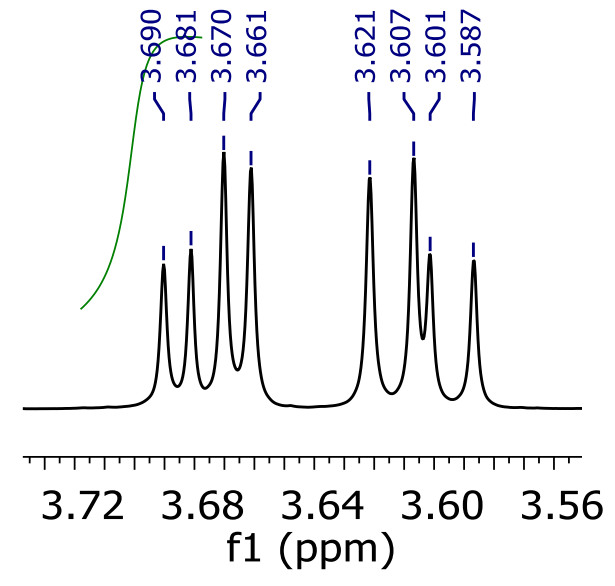

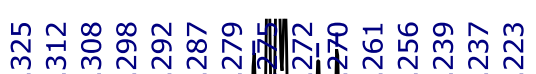
กิ
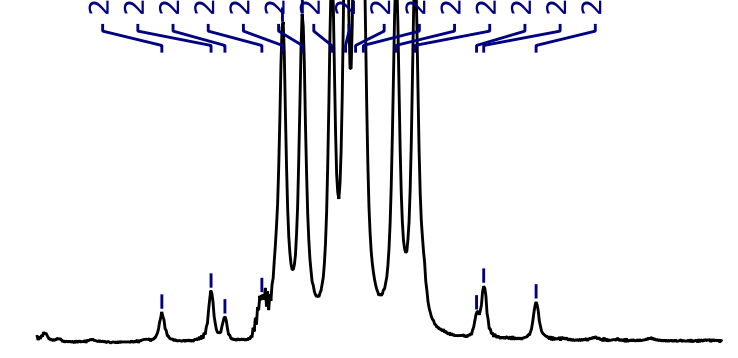

$\begin{array}{llllll}2.33 & 2.30 & 2.27 & 2.24 & 2.21 & 2.18\end{array}$ f1 (ppm)

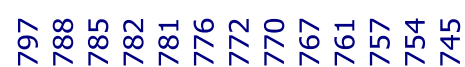

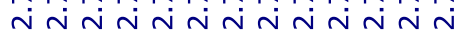

$\underbrace{111}$
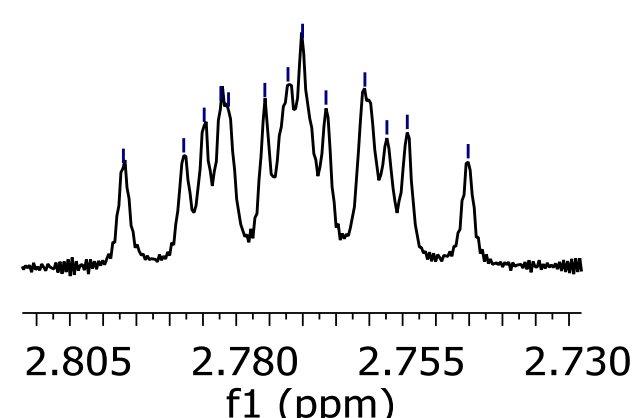

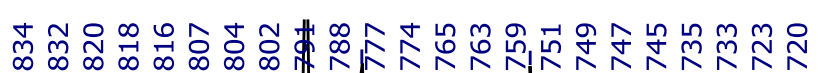

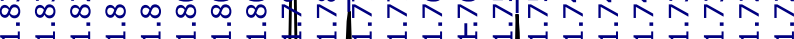

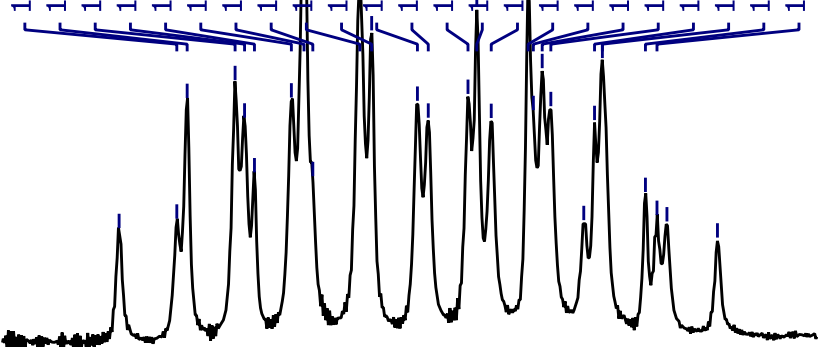

$\begin{array}{lllllll}1.87 & 1.84 & 1.81 & 1.78 & 1.75 & 1.72 & 1.69\end{array}$ f1 (ppm)

14



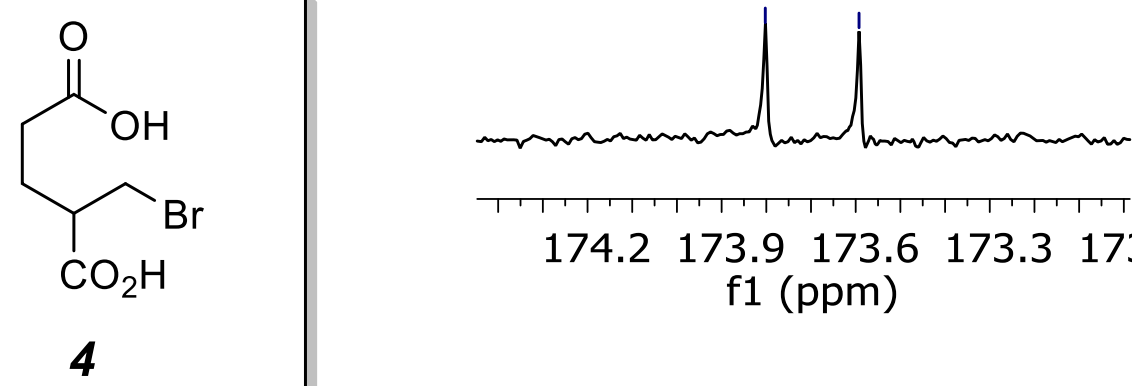

$\begin{array}{lllll}174.2 & 173.9 & 173.6 & 173.3 & 173.0\end{array}$

f1 (ppm)

$125 \mathrm{MHz}, \mathrm{CDCl}_{3}$

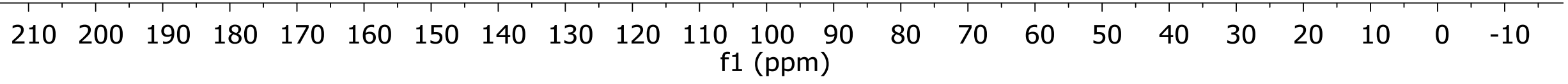




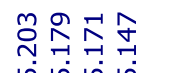

ñ

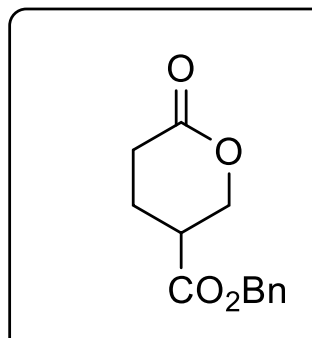

CBnVL

$500 \mathrm{MHz}, \mathrm{CDCl}_{3}$

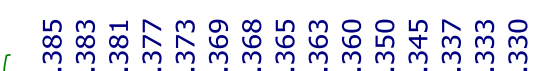
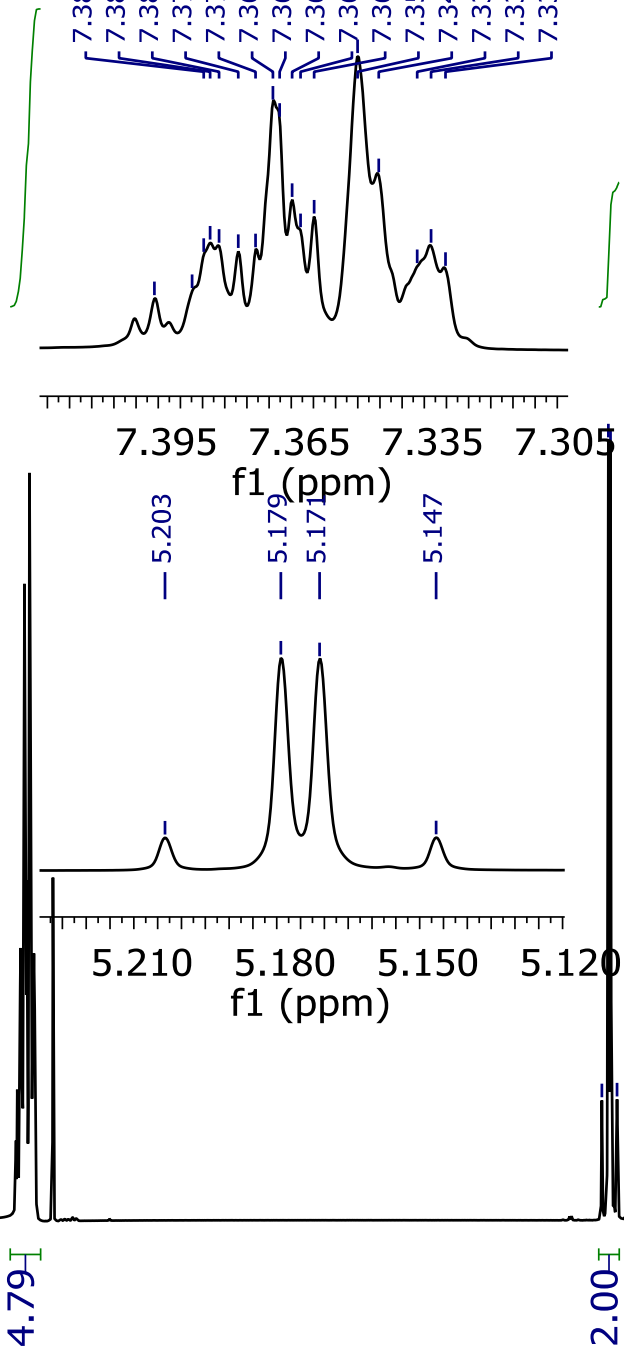

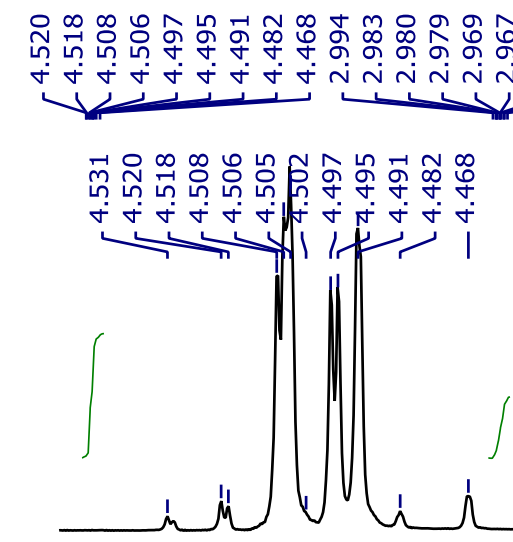

गामापाम

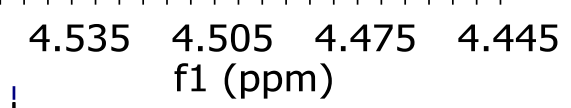

$$
\text { f1 (ppm) }
$$

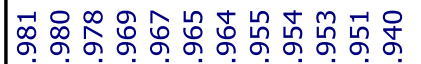
ن NiN $\underbrace{2}$
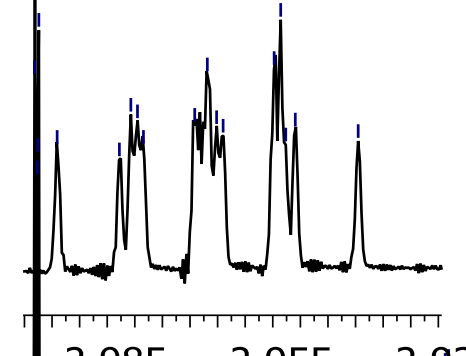

$2.985 \quad 2.955$

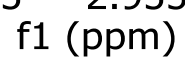

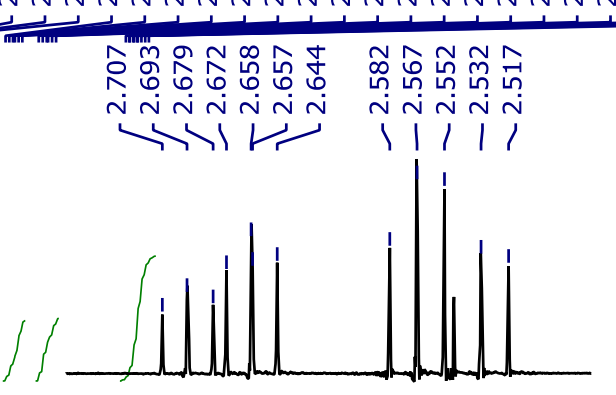

$\begin{array}{lllll}2.72 & 2.66 & 2.60 & 2.54 & 2.48\end{array}$ f1 (ppm)

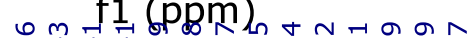

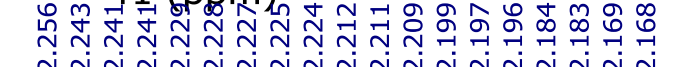

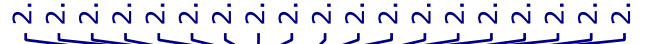

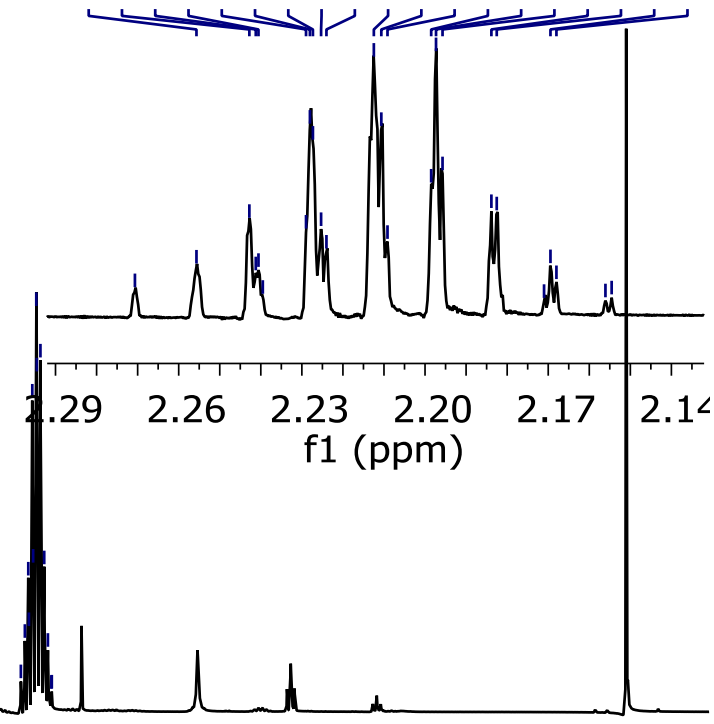

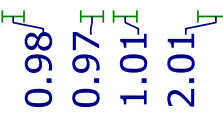

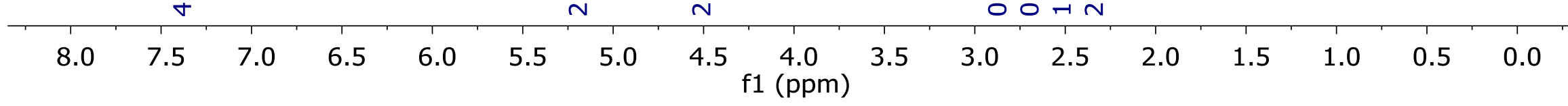



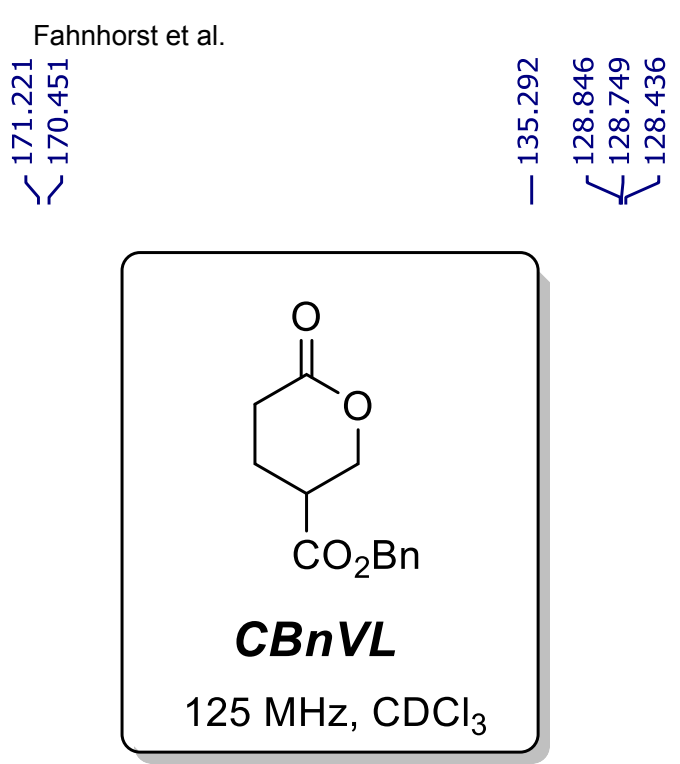

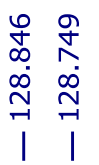

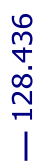

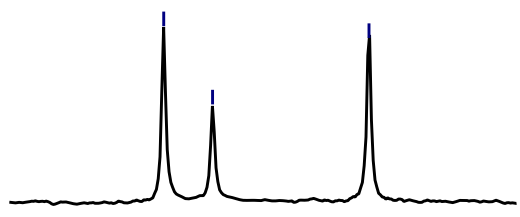

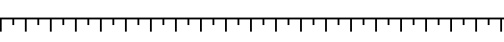

$\begin{array}{lll}128.85 & 128.50 \quad 128.15\end{array}$ f1 (ppm)

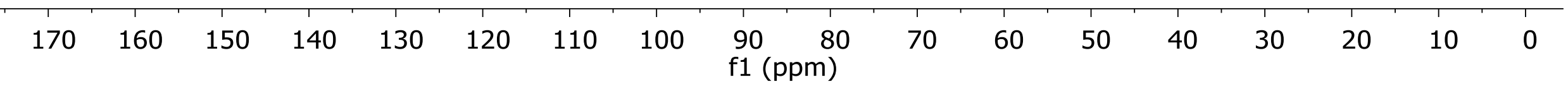



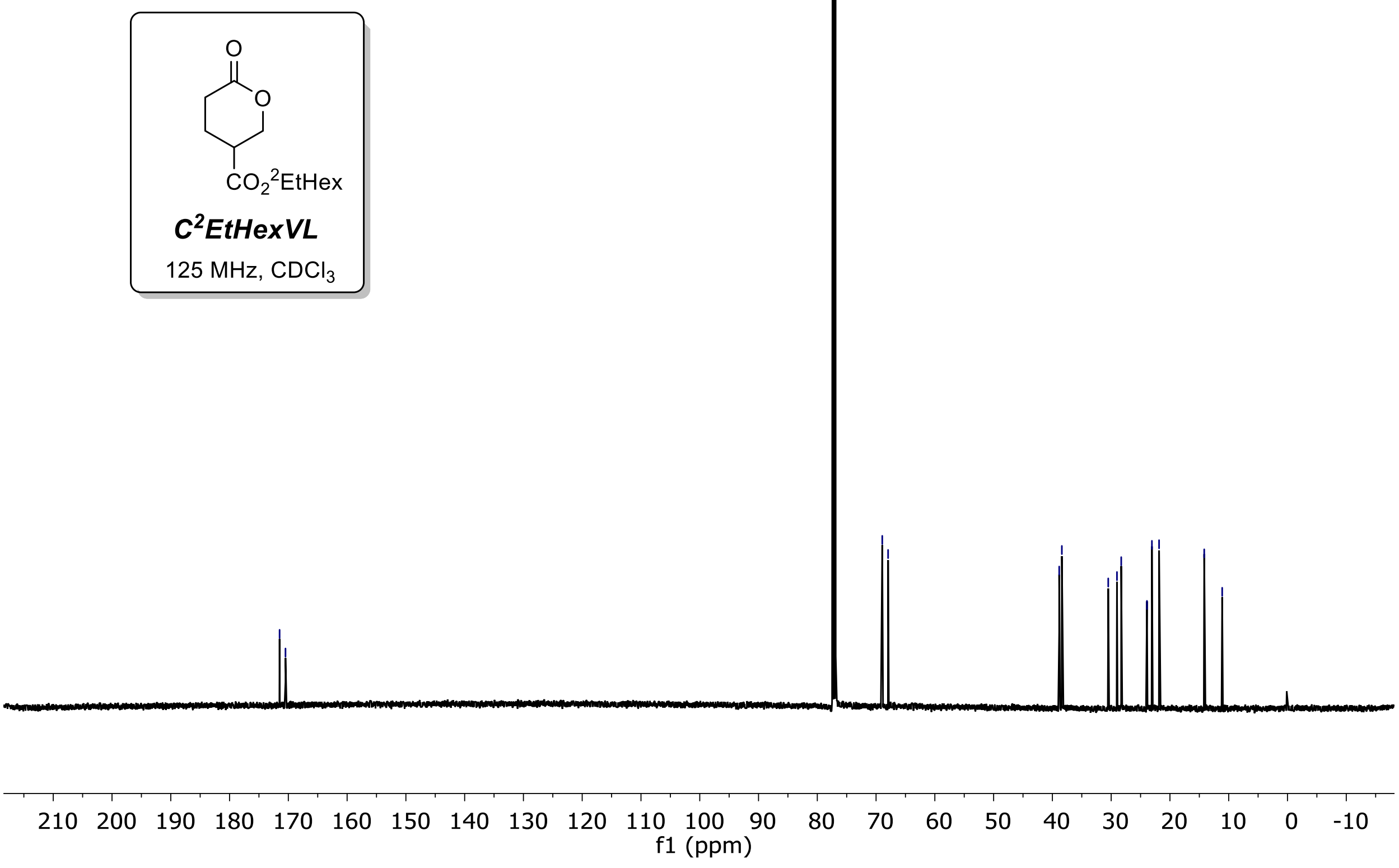

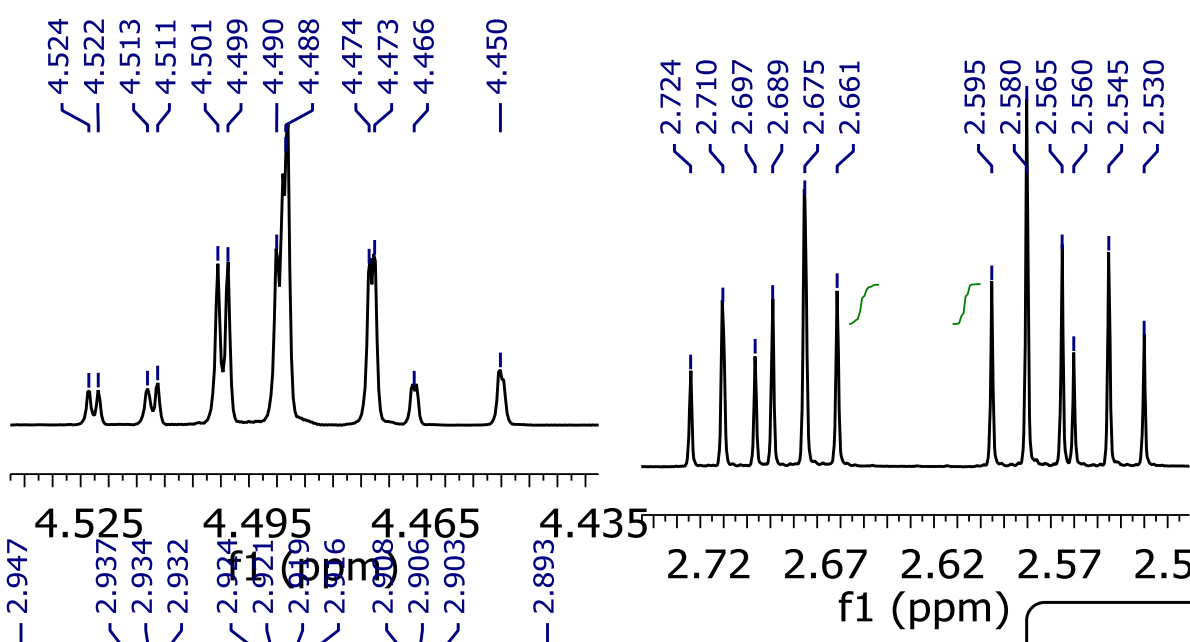

4.435

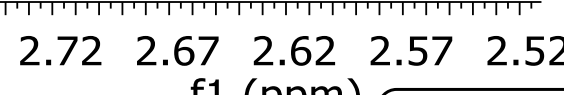

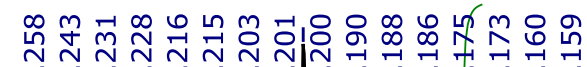

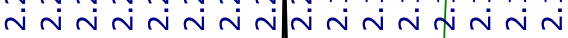

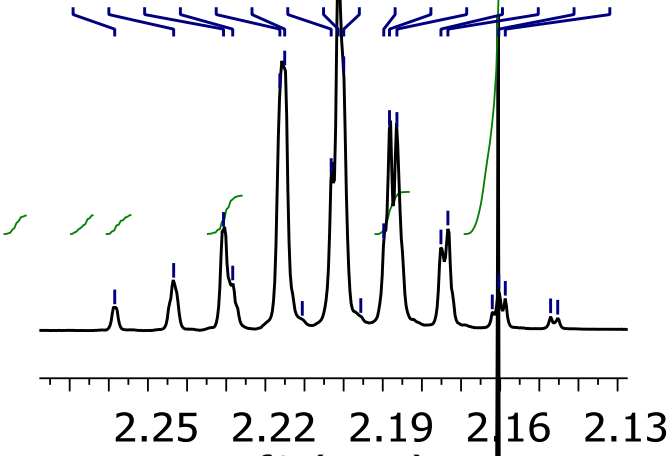

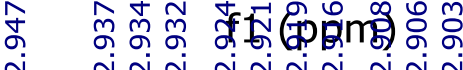

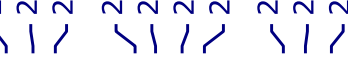
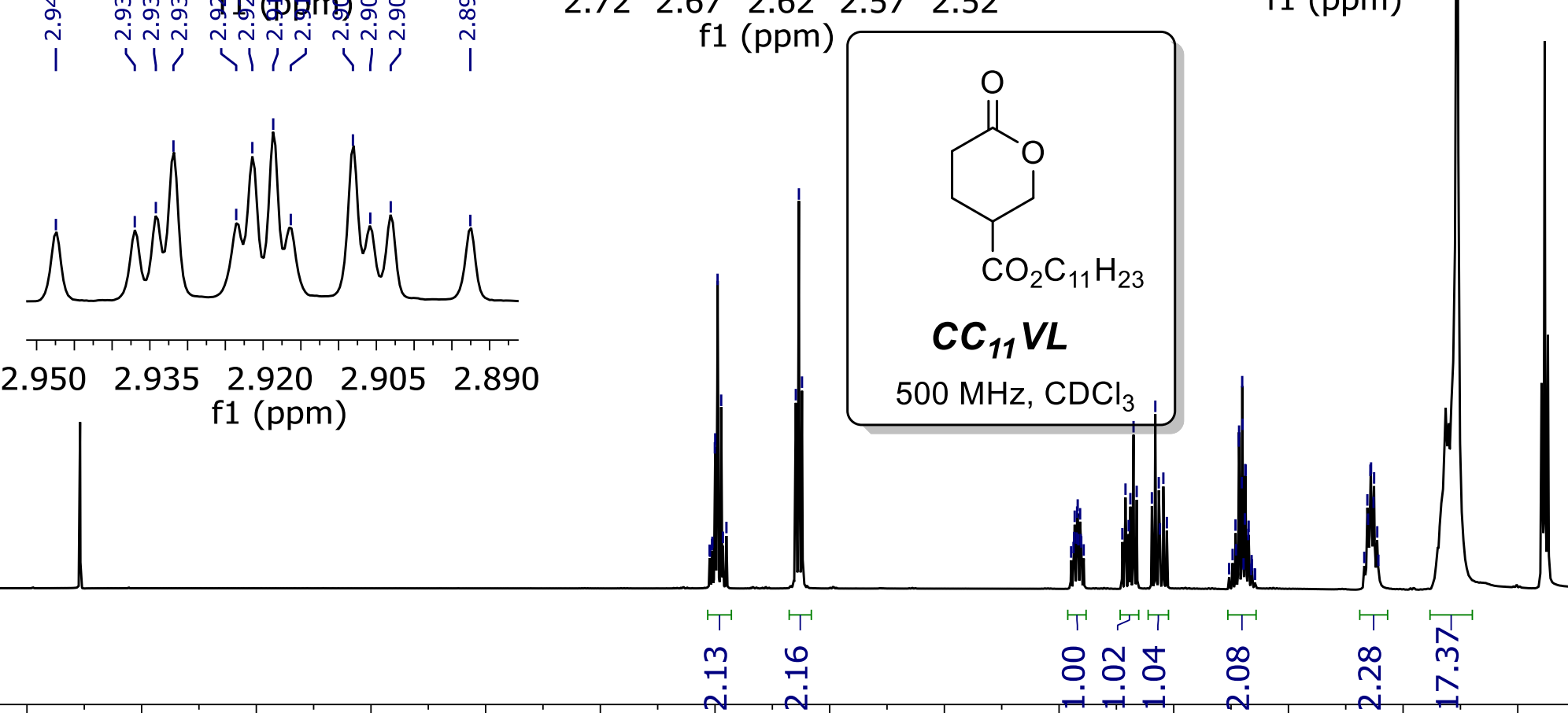

$7.5-7.0$

$6.5 \quad 6.0$

5.5

5.0

$\begin{array}{lll}4.5 & 4.0 & 3.5\end{array}$ f1 (ppm)

'T 田行

T

T

8 웅

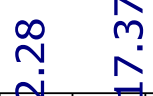

$\begin{array}{lllll}1.5 & 1.0 & 0.5 & 0.0 & -0\end{array}$ 

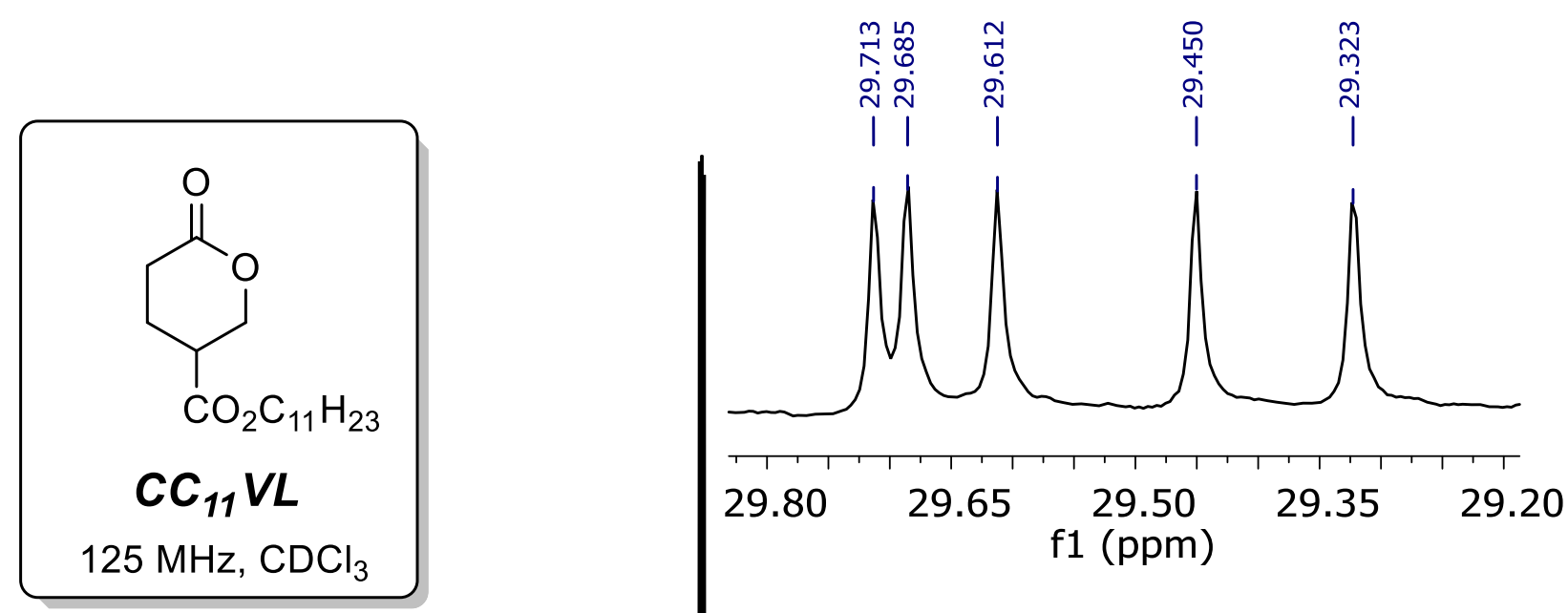

$\begin{array}{lllllllllllllllllllllll}210 & 200 & 190 & 180 & 170 & 160 & 150 & 140 & 130 & 120 & 110 & 100 & 90 & 80 & 70 & 60 & 50 & 40 & 30 & 20 & 10 & 0 & -10\end{array}$




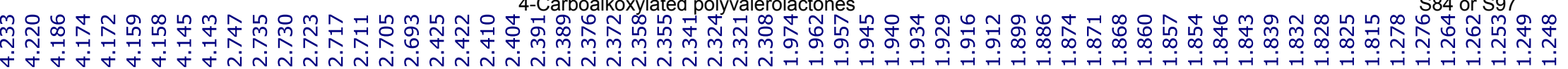
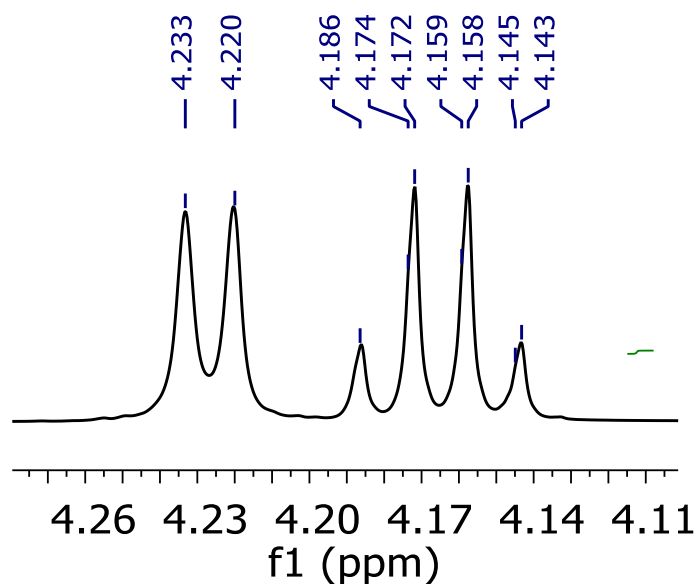
f1 (ppm)

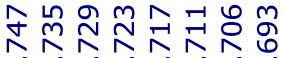

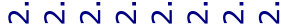
$<111111>$
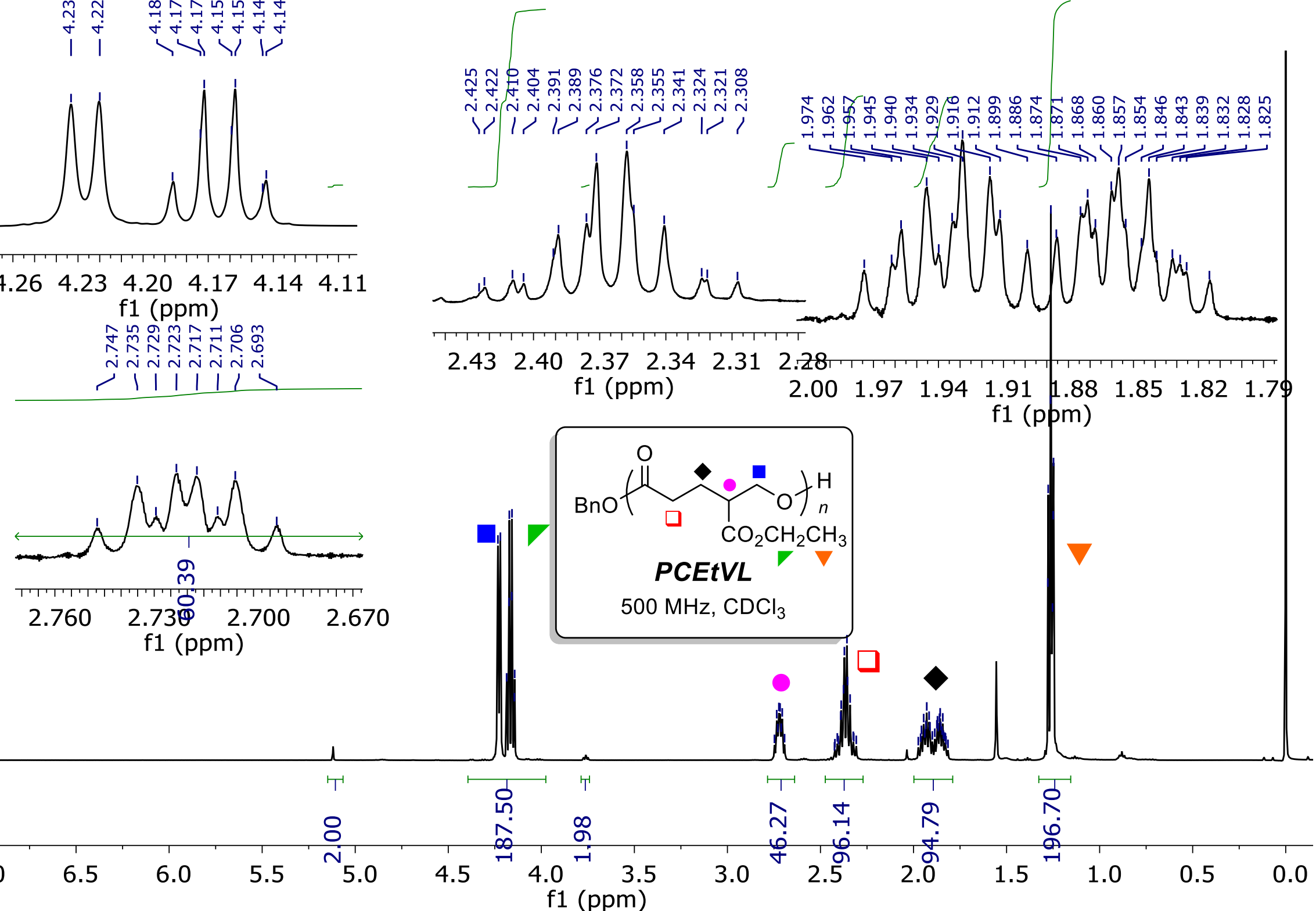
ì

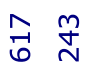

$\underset{1}{\stackrel{N}{N}}$

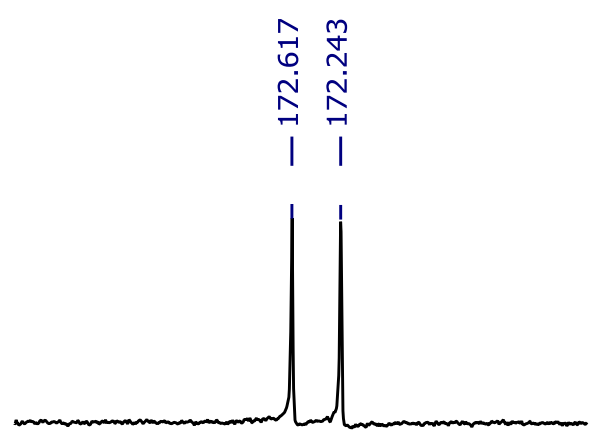

। 1

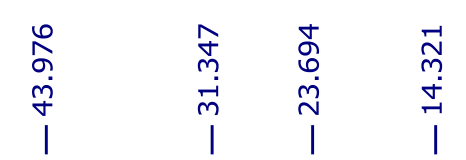
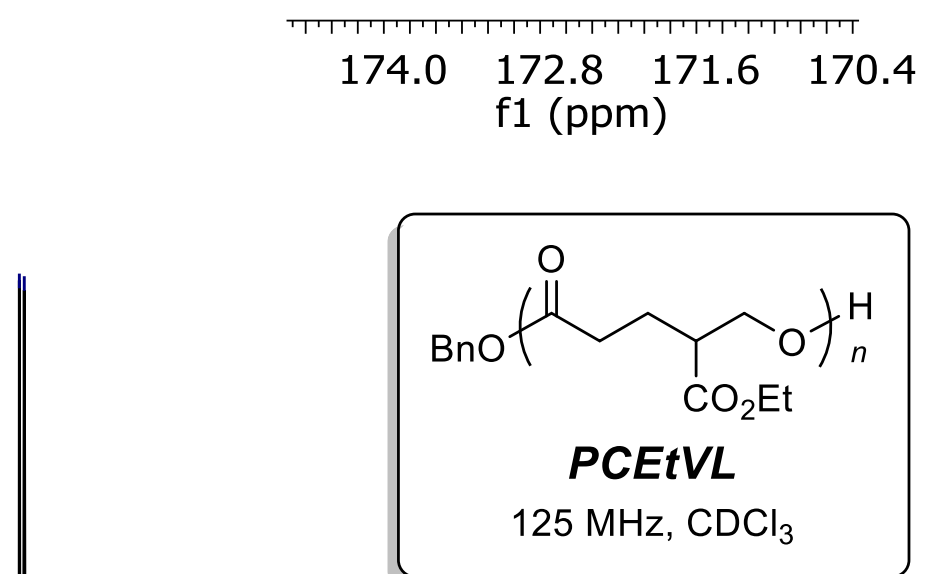

$\begin{array}{llllllllllllllllll}180 & 170 & 160 & 150 & 140 & 130 & 120 & 110 & 100 \underset{\mathrm{f} 1(\mathrm{ppm})}{90} & 80 & 70 & 60 & 50 & 40 & 30 & 20 & 10 & 0\end{array}$




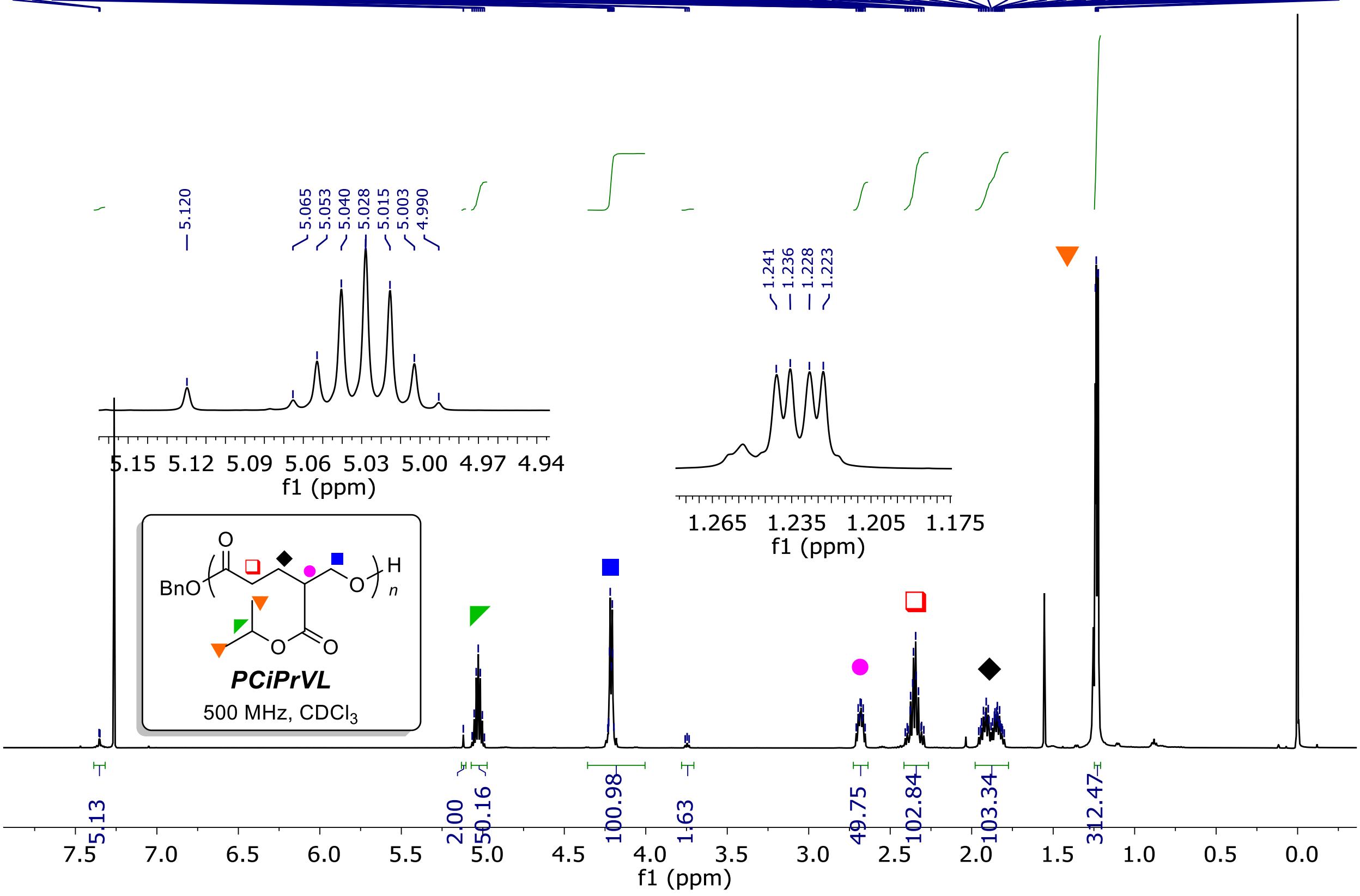


ํํํํำ

촉

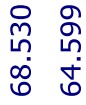

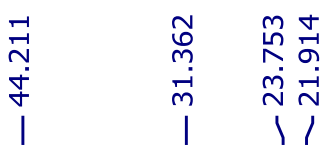

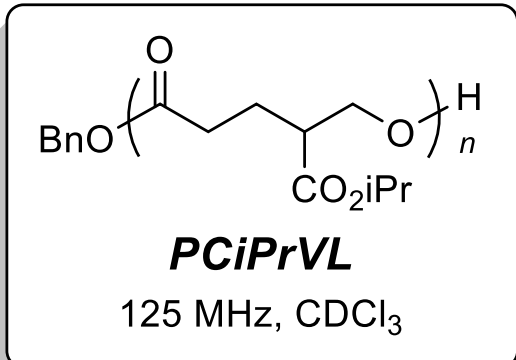




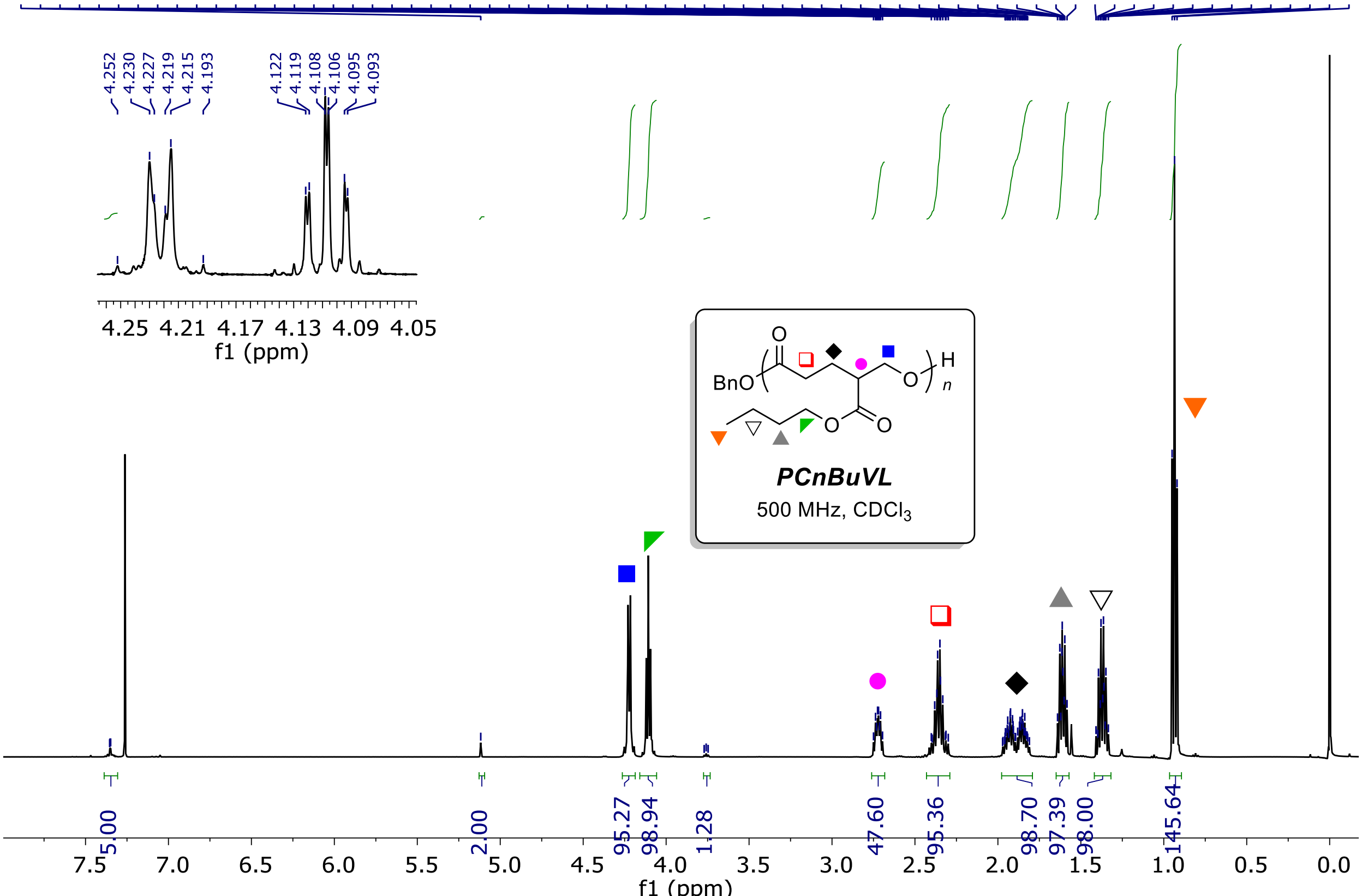
1 (ppm)

$$
\text { f1 (ppm) }
$$




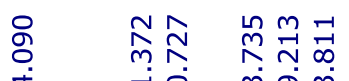

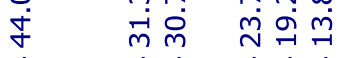

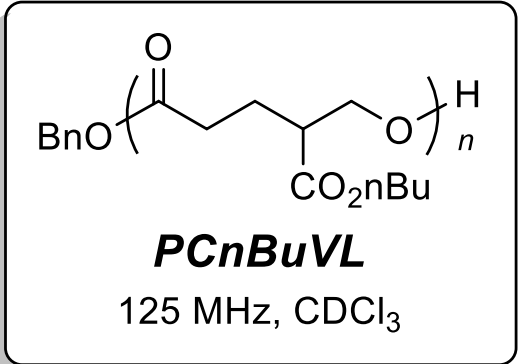

$\begin{array}{lllllllllllllllllllllll}210 & 200 & 190 & 180 & 170 & 160 & 150 & 140 & 130 & 120 & \begin{array}{c}110 \\ \mathrm{f} 1\end{array}\left(\begin{array}{ll}100 \\ (\mathrm{ppm})\end{array}\right. & 90 & 80 & 70 & 60 & 50 & 40 & 30 & 20 & 10 & 0 & -10\end{array}$




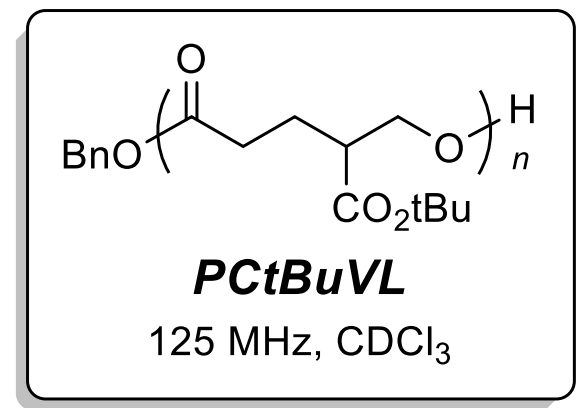

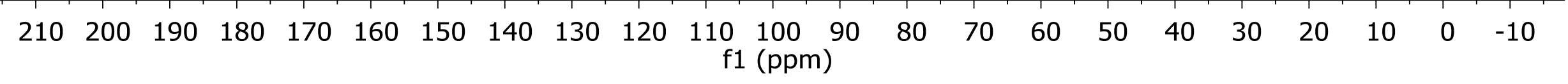




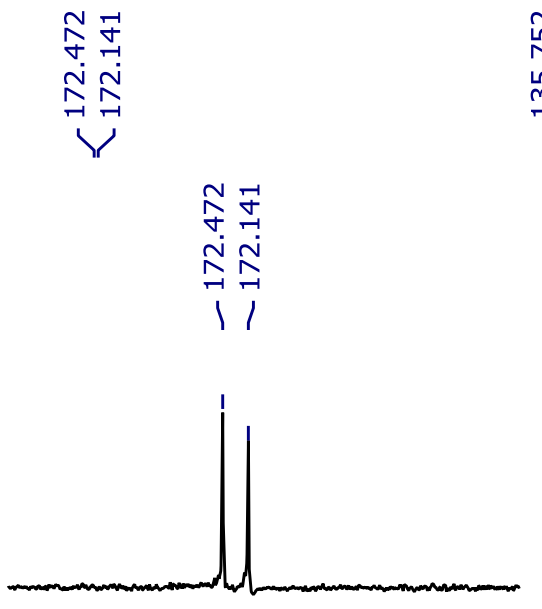

$\begin{array}{llll}175.0 & 173.0 & 171.0 & 169.0\end{array}$ f1 (ppm)
กิ กิํำ

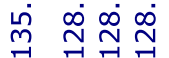

年

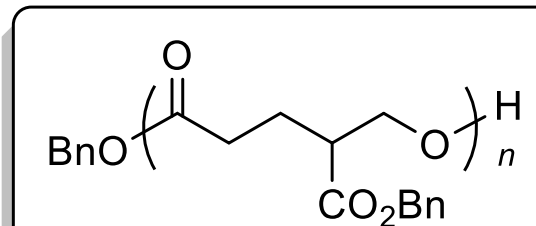

PCBnVL

$125 \mathrm{MHz}, \mathrm{CDCl}_{3}$

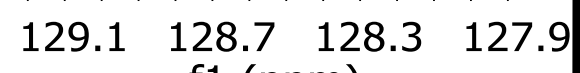
f1 (ppm)
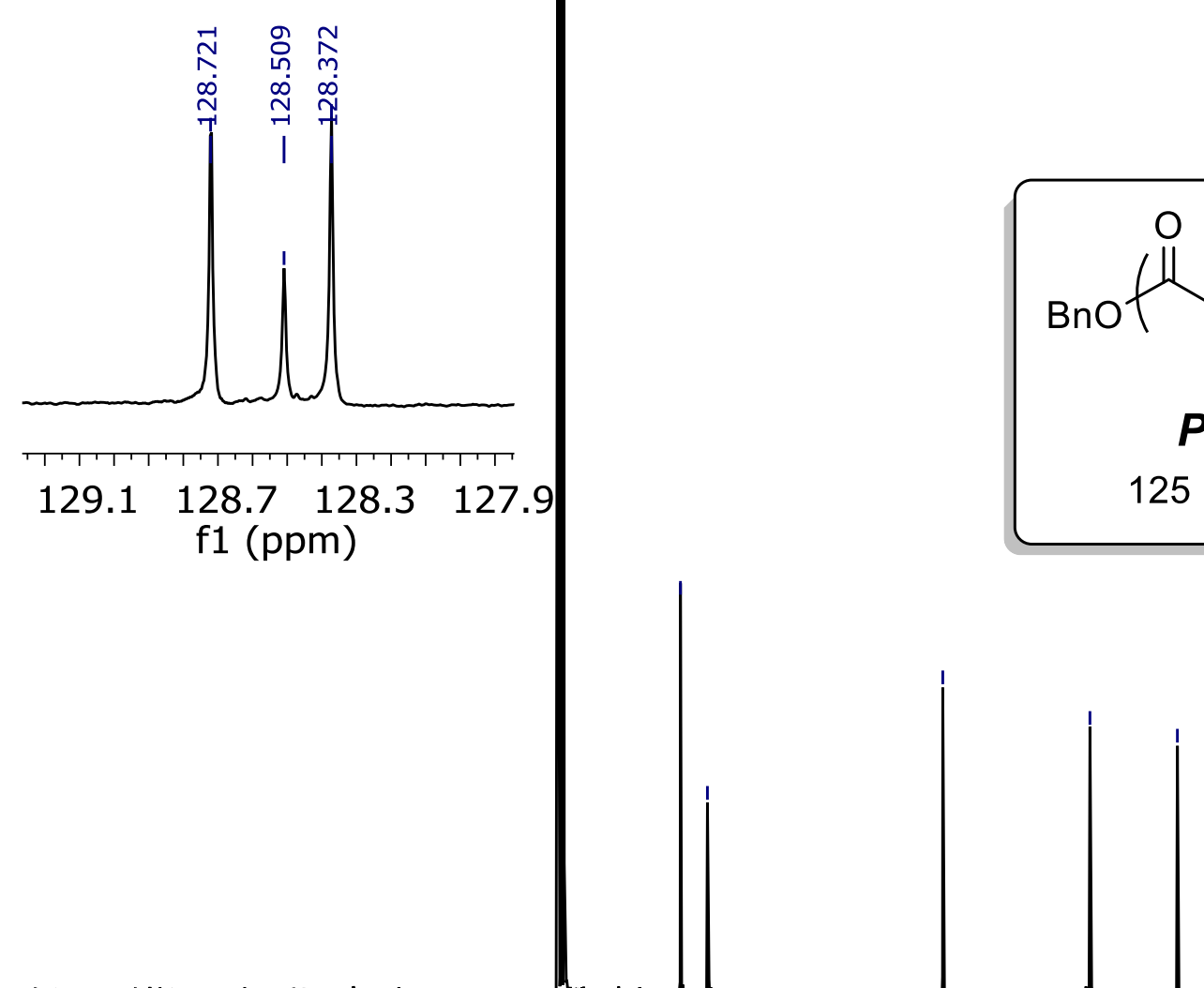


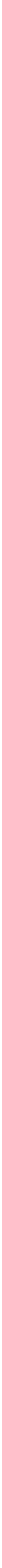



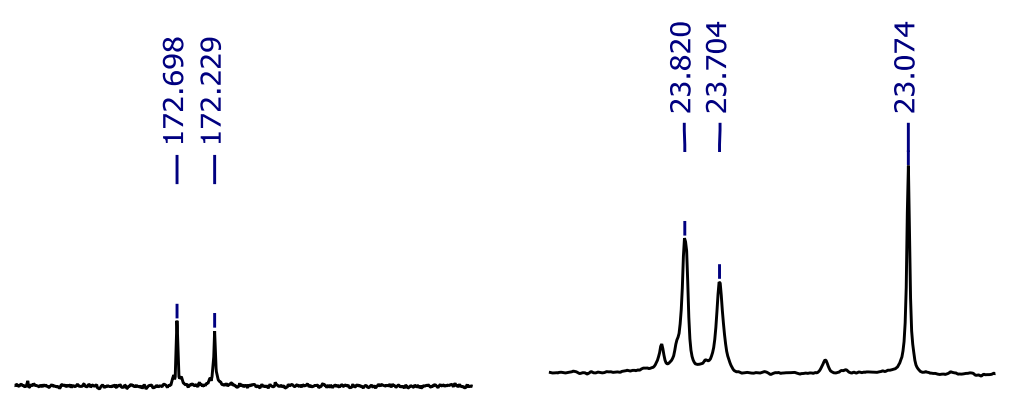

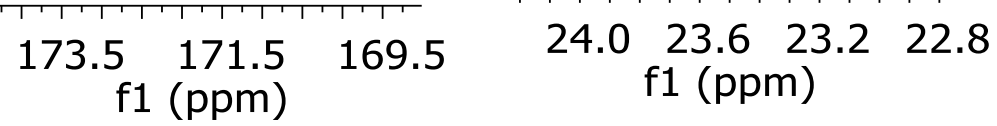

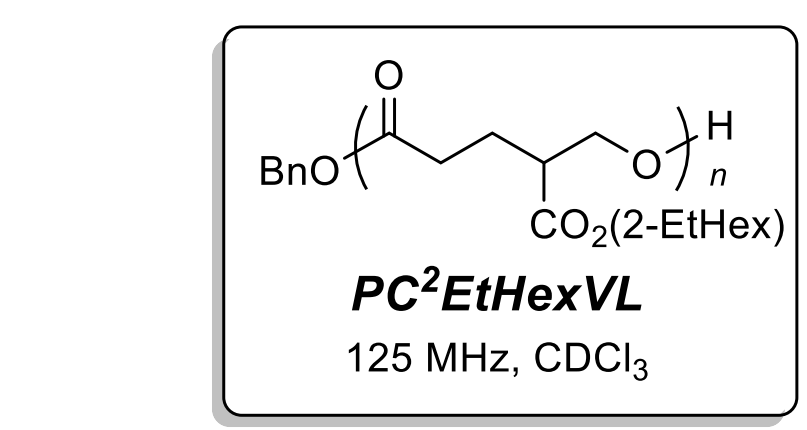

$$
\begin{array}{llllllllllll}
210 & 200 & 190 & 180 & 170 & 160 & 150 & 140 & 130 & 120 & 110 \quad \begin{array}{l}
100 \\
\mathrm{f} 1(\mathrm{ppm})
\end{array} & 90
\end{array}
$$




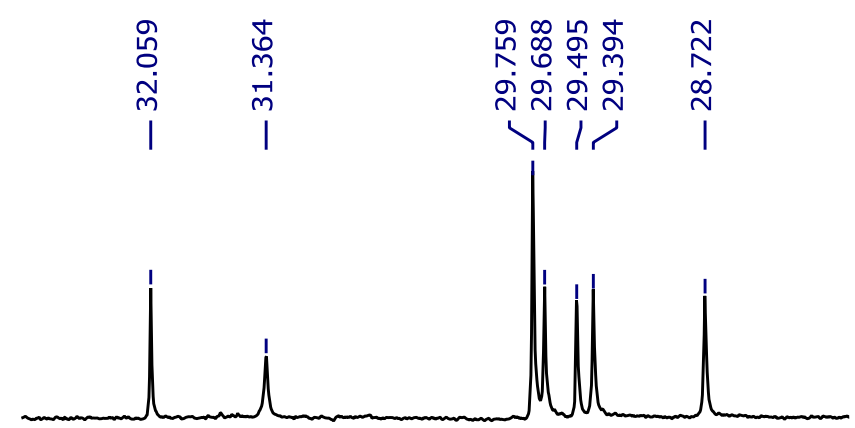

$\begin{array}{lllllll}32.8 & 32.0 & 31.2 & 30.4 & 29.6 & 28.8 & 28.0\end{array}$

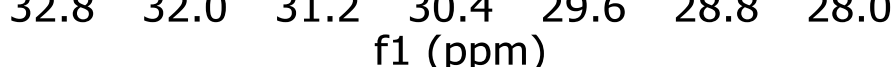

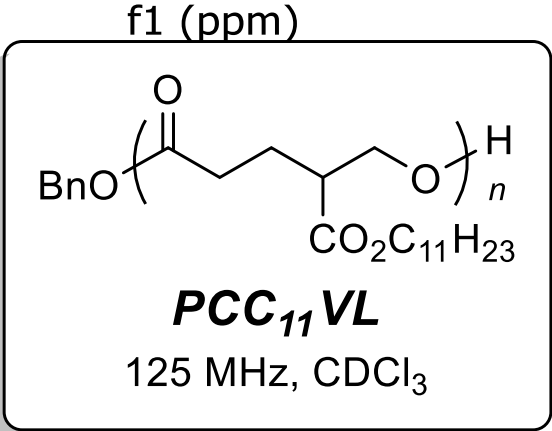

